\title{
The Broken Supersymmetry Phase of a Self-Avoiding Random Walk
}

\author{
Steven E. Golowich ${ }^{1, \star}$, John Z. Imbrie ${ }^{2, \star}$ \\ ${ }^{1}$ Harvard University, Cambridge, MA 02138, USA \\ 2 University of Virginia, Charlottesville, VA 22903, USA
}

Received 9 November 1993/in revised form: 11 March 1994

\begin{abstract}
We consider a weakly self-avoiding random walk on a hierarchical lattice in $d=4$ dimensions. We show that for choices of the killing rate $a$ less than the critical value $a_{c}$ the dominant walks fill space, which corresponds to a spontaneously broken supersymmetry phase. We identify the asymptotic density to which walks fill space, $\rho(a)$, to be a supersymmetric order parameter for this transition. We prove that $\rho(a) \sim\left(a_{c}-a\right)\left(-\log \left(a_{c}-a\right)\right)^{1 / 2}$ as $a \nearrow a_{c}$, which is mean-field behavior with logarithmic corrections, as expected for a system in its upper critical dimension.
\end{abstract}

\section{Introduction and Results}

The self-avoiding walk (SAW) has long been studied in the physics literature due to its significance as a model for physical polymers [dG2, dCJ]. Recently it has received attention from a rigorous perspective as well [MS, BI, IM]. Most of the rigorous work has been directed towards establishing the properties of either fixed-length walks in the presence of a strictly repulsive interaction or the Green's function of such a process at or above the critical point. In this paper, however, we study a SAW in the so-called dense phase, where the dominant paths fill space to some nonzero mean density. We work in $d=4$ dimensions, which is the borderline between simple mean-field behavior $(d>4)$ and complex behavior $(d<4)$. A consequence of this is that the critical behavior is modified slightly from mean-field, but is still tractable. For a weakly self-avoiding walk on a hierarchical lattice we rigorously calculate the critical behavior of the density, finding the leading powerlaw behavior to be mean-field, but with logarithmic corrections.

The model we study is essentially the same as the one introduced in [BEI, BI], so we will only briefly describe it here. By a hierarchical lattice $\mathscr{G}$ we mean the direct sum of infinitely many copies of $\mathbb{Z}_{L^{4}}$, with $L$ some positive integer. A point

\footnotetext{
${ }^{\star}$ Research partially supported by NSF Grants DMS 91-2096 and DMS 91-96161.
} 
$x \in \mathscr{G}$ can be written as a sequence(..., $\left.x_{2}, x_{1}, x_{0}\right)$, with $x_{i} \in \mathbb{Z}_{L^{4}}$, and all but finitely many $x_{i}$ being zero. The hierarchical norm we use is a $\mathscr{G}$-invariant ultrametric defined by

$$
|x| \equiv \begin{cases}0 & \text { if } x=(\ldots, 0) \\ L^{N} & \text { if } x=\left(\ldots, 0, x_{N-1}, \ldots, x_{0}\right) \text { and } x_{N-1} \neq 0 .\end{cases}
$$

Our SAW is a perturbation of a Lévy process on $\mathscr{G}$ chosen so that the free Green's function $G(x-y)=|x-y|^{-2}$ for $x \neq y$. Details on the process are given in Sect. 2 . We work in a finite volume $\Lambda$ by killing the process when it first exists $\Lambda$. We denote by $E_{0, \Lambda}$ the expectation for this killed process.

We measure the amount of self-intersection inside $\Lambda$ by

$$
\tau^{2}(\Lambda) \equiv \int d s d t \mathbf{1}_{\{\omega(s)=\omega(t) \in \Lambda\}},
$$

where $\omega(t)$ is a sample path for the process. We define the interacting Green's function by

$$
G_{\lambda, \Lambda}(a, x, y)=\int_{0}^{\infty} d T e^{-a T} E_{0, \Lambda}\left(e^{-\lambda \tau^{2}(\Lambda)} \mathbf{1}_{\omega(T)=y} \mid \omega(0)=x\right) .
$$

This function was studied in [BI]. They found that, in the infinite-volume limit, there exists a critical value $a_{c}=a_{c}(\lambda)$ such that $G_{\lambda}\left(a_{c}, 0, x\right)=O\left(|x|^{-2}\right)$ as $x \rightarrow \infty$. In other words, the model exhibits massless decay at $a=a_{c}$. They also constructed the Green's function at values $a>a_{c}$, finding there the hierarchical version of exponential decay. We think of our model as being comprised of an ensemble of walks of all different lengths, with each walk weighted according to its length (the $e^{-a T}$ term) and its self-interactions (the $e^{-\lambda \tau^{2}}$ term). When the killing rate $a$ is larger than critical value, only short walks contribute, hence the rapid decay of the correlations. As $a \searrow a_{c}$, walks of all lengths contribute, resulting in slow power-law decay.

We are interested in studying the case of $a<a_{c}$. Here it is crucial to work in a finite volume with a self-avoiding interaction; with these two constraints, we would heuristically expect walks to fill the volume, encouraged by the negative killing rate, but then to stop at some finite density, discouraged by the interaction. In fact this is what happens and, furthermore, after taking the infinite volume limit we find a phase transition at $a=a_{c}$ between the massive phase studied in [BI] and this dense phase. The density $\rho(a)$ is an order parameter, being zero above the transition and nonzero below. We make this more precise below.

In order to simplify the construction we condition on walks beginning and ending at the origin (though we could also consider more general walks). We define the expectation for such walks

$$
E_{a, \lambda, \Lambda}(F(\vec{\tau})) \equiv \frac{\int_{0}^{\infty} d T F\left(\vec{\tau}^{T}\right) e^{-a T} E_{0, \Lambda}\left(e^{-\lambda \tau^{2}(\Lambda)} \mathbf{1}_{\omega(T)=0} \mid \omega(0)=0\right)}{G_{\lambda, \Lambda}(a, 0,0)},
$$

where we have defined $\vec{\tau}^{T}=\left\{\tau_{x}^{T}\right\}_{x \in \Lambda}$, and $\tau_{x}^{T}$ is the local time the walk spends at site $x$, defined as

$$
\tau_{x}^{T} \equiv \int_{0}^{T} d s \mathbf{1}_{\{\omega(s)=x\}}
$$


Since the local time is a measure of the density of the walk at a site our order parameter will involve an expectation of the local time. Notice that $\lim _{\Lambda \rightarrow \infty} E_{a, \lambda, \Lambda}\left(\tau_{x}\right) \neq 0$ for any fixed $x$ in either phase. However, from the analysis in $[\mathrm{BI}]$ it is possible to show

$$
\lim _{x \rightarrow \infty} \lim _{\Lambda \rightarrow \infty} E_{a, \lambda, \Lambda}\left(\tau_{x}\right)=0 \text { for } a \geqq a_{c} .
$$

So we choose this as our order parameter:

$$
\rho(a) \equiv \lim _{x \rightarrow \infty} \lim _{\Lambda \rightarrow \infty} E_{a, \lambda, \Lambda}\left(\tau_{x}\right) .
$$

Our main result is to find the behavior of $\rho(a)$ for a less than $a_{c}$. We prove

Theorem 1.1. Let $d=4$, and choose some $L$ sufficiently large, then $\lambda>0$ sufficiently small. Then for each $\beta>0$, sufficiently small,

$$
\rho\left(a_{c}(\lambda)-\beta\right)=U \beta(-\log \beta)^{1 / 2}\left(1+O\left((-\log \beta)^{-1 / 2}\right)\right),
$$

$U>0$ is a constant that may depend on $L$ and $\lambda$.

Note that the leading term is mean-field behavior with logarithmic corrections, as one would expect for a system in its upper critical dimension.

The dense phase for a SAW we first discussed by Parisi and Sourlas [PS]. Also in that paper they introduced the idea of studying a SAW as a supersymmetric field theory, independently proposed by McKane [M], which is the method we use. Dense polymers in two dimensions, both linear and branched, have been extensively studied by Duplantier and Saleur; see [DS] and references therein. The phase diagram of $\phi_{4}^{4}$ theories in the presence of a field $h$, for $\phi$ an $n$-vector with $n<1$, and its relation to polymer theory have also been studied [GS, WSPP].

An important question is whether our techniques could be extended to the case of a non-hierarchical walk. As we will see, the bosonic part of the model resembles a $\sigma$-model. This fact, especially the presence of Goldstone modes, would considerably complicate the analysis in the non-hierarchical case. While considerable progress on such issues has been made by Balaban [B] in the context of bosonic models at low temperature, the critical region still presents problems.

In three dimensions, self-interacting walks with two-body attractive and threebody repulsive interactions are used to model physical polymers in poor solvents [dCJ]. Near the $\theta$ compensation point the theory is believed to exhibit tricritical behavior. Also near this point fixed-length polymers undergo a collapse transition [dG1, OPB]. We believe the techniques developed in this paper, suitably extended, could shed some light on these problems.

We will now outline the proof of Theorem 1.1. The principle tool is the renormalization group applied to the supersymmetric field theory representation of the SAW model. We begin in Sect. 2 by describing the Lévy process on the hierarchical lattice, and show how to compute SAW expectations by evaluating certain Berezin integrals, which, in the language of physics, are correlation functions of a $\lambda \Phi^{4}$ hierarchical lattice field theory, where the killing rate $a$ plays the role of the (mass) $)^{2}$. Here the fields are superfields $\Phi=(\varphi, \bar{\varphi}, \psi, \bar{\psi})$, with the first two components comprising a bosonic (commuting) complex scalar field and the least two being fermionic (anticommuting, or Grassmann) fields. Because the action is a function only of the square $\Phi^{2} \equiv \varphi \bar{\varphi}+\psi \bar{\psi}$, the theory is invariant under 
transformations of the fields leaving $\Phi^{2}$ invariant. This is the supersymmetry, which we will comment on further below.

In Sect. 3 we set up the renormalization group framework we will be using to compute the Berezin integrals. Our treatment in these two sections is brief since extended explanations already exist in [BEI, BI]. In Sect. 4 we prove some analytical lemmas useful for keeping track of remainders during the induction.

The main body of the paper begins with Sect. 5, where we consider the action of the renormalization group map on the self-avoidance interaction. This consists of following the evolution of the effective potential $v(\Phi)$ as we apply the mapping repeatedly. The initial form of the potential is

$$
v(\Phi)=\lambda \Phi^{4}+\left(a_{c}-\beta\right) \Phi^{2}
$$

with $a_{c}=-O(\lambda)$. Because we are interested in the behavior near the critical point, we start with $\beta$ small, and hence the initial $v$ has the form of a shallow "Mexican hat." While $\beta$ is small, it essentially grows by a factor of $L^{2}$ under each step of the $\mathrm{RG}$ map. This simply reflects the fact that the mass is a relevant parameter, according to the renormalization group, and so is driven away from the fixed-point value. So the Mexican hat becomes deeper the longer we flow under the RG. While we are still near the critical trajectory, the techniques of [BEI, BI] apply with little modification, but once we get significantly into the deep Mexican hat region we must develop new methods that take the new shape of $v$ into account. Here the model starts to look like a $\sigma$-model, and in fact we have used ideas developed to study the hierarchical version of that model [GK].

In Sect. 6 we apply the RG map to those blocks containing observables. In Sect. 7 we assemble the results of the previous sections into an expression for $E_{a, \lambda, \Lambda}\left(\tau_{z}\right)$ which is a ratio of two one-dimensional integrals, the results of applying the RG sufficiently many times so the volume has been reduced to a single point. These integrals are easily evaluated, and then the limits $\Lambda, z \rightarrow \infty$ may be taken, yielding the result of Theorem 1.1.

Finally, we comment on the nature of the phase transition and of the order parameter $\rho$. In the spin system representation, we noted that the model exhibits a supersymmetry, and that the killing rate becomes the (mass) ${ }^{2}$. Because we have set $a$ smaller than the critical value, we see we are in the low-temperature phase of the spin system. We thus expect to see consequences of a broken symmetry (in this case, a broken supersymmetry). The usual order parameter for such a transition is the magnetization, or expectation of a single field component $\langle\Phi\rangle$. However, this would not be relevant for the SAW model, because only the square of a field $\Phi^{2}$ has significance (local time) in the SAW representation. But measuring $\left\langle\Phi^{2}\right\rangle$, where $\langle\cdot\rangle$ is a spin-system expectation, would always give the result zero, by supersymmetry (cf. [BEI] Theorem 4.2). By contrast, in an $n$-vector (bosonic) magnetic system where $n>0 .\langle\vec{\phi} \cdot \vec{\phi}\rangle$ is always non-zero, being the expectation of a nonnegative quantity. Our model parameter $\rho$ avoids both of these extremes because, in the spin system representation, it includes the square of a superfield (local time, but also two individual components of the field, corresponding to the beginning and endpoint of the walk: $\left\langle\varphi_{0} \bar{\varphi}_{0} \Phi_{z}^{2}\right\rangle$. In the limit $z \rightarrow \infty, \rho(a)=0$ for $a \geqq a_{c}$, which is a consequence of the unbroken supersymmetry: i.e. $\left\langle\varphi_{0} \bar{\varphi}_{0} \Phi_{z}^{2}\right) \stackrel{z \rightarrow \infty}{\longrightarrow}\left\langle\varphi_{0} \bar{\varphi}_{0}\right\rangle\left\langle\Phi_{z}^{2}\right\rangle$ in a massive theory. When $a<a_{c}, \rho(a) \neq 0$, and we are seeing a consequence of the broken supersymmetry: $\rho$ is a way of measuring an expectation of $\Phi^{2}$ that does not trivially vanish. 


\section{The Process}

We will first construct the free (noninteracting) process, and then explain how to add self-avoidance. It is a similar process to that used in [BEI] but differs in two ways. First, the boundary conditions are different; we work in finite volume $\Lambda$, with walks that are killed on first exit from $\Lambda$. This is also done in [BI]. Second, we use a slightly different probability density $q(x)$ for the steps of the walk. This is done for technical reasons, which we discuss later, and which are related to working in the broken phase. The difference between this process and that used in [BI] is minor; the short distance behavior of $q(x)$ is modified slightly, but it is the same in the limit of large $x$.

We now recall some notation from $[\mathrm{BEI}]$ and construct our Lévy process. We denote by $\mathscr{G}$ the hierarchical lattice, so $\mathscr{G}=\bigoplus_{k=0}^{\infty} \mathbb{Z}_{n}, n=L^{4}$. We define the subgroups

$$
\begin{aligned}
& \{0\}=\mathscr{G}_{0} \subset \mathscr{G}_{1} \subset \cdots \subset \mathscr{G}, \\
& \mathscr{G}_{k}=\left\{x \in \mathscr{G}: x_{i}=0, i \geqq k\right\} .
\end{aligned}
$$

We use the hierarchical norm on $\mathscr{G}$, defined to be

$$
|x|= \begin{cases}0 & \text { if } x=0 \\ L^{p}, p=\inf \left\{k: x \in \mathscr{G}_{k}\right\} & \text { if } x \neq 0 .\end{cases}
$$

Let $\mathscr{H}$ be the dual to the group $\mathscr{G}$, so $\mathscr{H}=\times_{k=0}^{\infty} \widehat{\mathbb{Z}}_{n}$. Also define $\mathscr{H}_{k}$ to be the annihilator of $\mathscr{G}_{k}$, so

$$
\mathscr{H}_{k}= \begin{cases}\left\{\left(\xi_{0}, \xi_{1}, \ldots\right): \xi_{i}=0 \text { if } i<k\right\} & \text { if } k>0 \\ \mathscr{H} & \text { if } k=0 .\end{cases}
$$

We define a norm on $\mathscr{H}$ to be

$$
|\xi|= \begin{cases}0 & \text { if } \xi=0 \\ L^{-p}, p=\sup \left\{k: \xi \in \mathscr{H}_{k}\right\} & \text { if } \xi \neq 0 .\end{cases}
$$

We define the free (noninteracting) process $\omega(t)$ in infinite volume (i.e. on $\mathscr{G}$ ) to be one that has probability $r d t$ of making a jump in time $[t, t+d t]$ and, given a jump, probability $q(x-y)$ of jumping from $x$ to $y$. We choose

$$
q(x)=\mathbf{1}_{\mathscr{G}_{0}}-\sum_{k=0}^{\infty} \frac{L^{-4 k}}{\left(L^{2 k}-L^{-2}\right)}\left(\mathbf{1}_{\mathscr{G}_{k}}-L^{-4} \mathbf{1}_{\mathscr{G}_{k+1}}\right),
$$

with

$$
\kappa \equiv\left(1-L^{-2}\right)^{-1}-L^{2}\left(1-L^{-2}\right) \sum_{k=1}^{\infty} \frac{L^{-6 k}}{\left(1-L^{-2 k}\right)\left(1-L^{-2(k+1)}\right)} .
$$

It is not hard to see that $q(x)$ is positive semi-definite, satisfies $\int_{\mathscr{G}} q(x) d x=1$, and $q(0)=0$. Also, $|q(x)| \sim|x|^{-6}$ as $x \rightarrow \infty$.

Before introducing the details of the finite-volume process we will briefly indicate the motivation for this choice of $q(x)$. We define the Green's function

$$
G(x, y)=G(x-y)=\int_{0}^{\infty} P(x-y, t),
$$

where $P(x, t)$ is the probability of finding the walk at site $x$ at time $t$. 
Lemma 2.1. A Lévy process on $\mathscr{G}$ which jumps according to (2.1), with jumping rate $r=1$, satisfies

$$
G(x)=\left\{\begin{array}{ll}
\frac{1}{|x|^{-2}} & \text { if } x=0 \\
\text { if } x \neq 0
\end{array} .\right.
$$

Proof. Fourier analysis on $\mathscr{G}$, exactly as in [BEI], Proposition 2.3.

Recall that a simple random walk on a Euclidean lattice has a Green's function that decays like $|x|_{E}^{2-d}$. Because our process on the hierarchical lattice has a Green's function with the same decay (measured with a hierarchical norm), we interpret our walk as the hierarchical version of the simple random walk.

We can rewrite $G$ as

$$
\begin{aligned}
& G(x)=\sum_{k=0}^{\infty} L^{-2 k} \Gamma\left(x / L^{k}\right), \\
& \Gamma(x)=\left(1-L^{-2}\right) \mathbf{1}_{|x|=0},
\end{aligned}
$$

i.e., $G(x-y)$ is a sum of rescaled copies of a matrix $\Gamma(x-y)$. Recall that, in the renormalization group scheme used in $[\mathrm{BEI}]$ and here the functions $\Gamma$ become the covariance matrices of the fluctuation convolutions (see Sect. 3). With this choice of $q(x)$, the matrix $\Gamma(x-y)$ is proportional to the identity matrix; in particular, it is nonsingular. This was not the case in [BEI]; they had a singular $\Gamma$ that annihilated functions with zero mean on a block. The nonsingularity of our $\Gamma$ is important for reasons that will become clear later; roughly, it means that our radial mass will flow to some fixed point under the renormalization group, rather than off to infinity, and it happens that our method requires this feature. We chose this particular nonsingular matrix (the identity) because it is the most convenient to handle technically (it is the same choice used in [GK]). We note that, with an appropriate choice of renormalization group transformation (RGT), one can always obtain a nonsingular $\Gamma$ for a non-hierarchical model. In the hierarchical case, the definition of the RGT is tied up with the definition of the model. This is why we need to change the model slightly in order to perturb $\Gamma$.

In this paper we will be using a different process $\omega_{N}(t)$, which is the same as the one we have described killed on first exit from some volume $\Lambda$. We assume $\Lambda$ to be a ball $\mathscr{G}_{N}$, for some $N \geqq 0$. Let $E_{0, N}$ denote the expectation for this process.

Lemma 2.2 (Lévy Hinčin formula).

$$
E_{0, N}\left(\left\langle\omega_{N}(t), \xi\right\rangle\right)=e^{-t \psi_{N}(\xi)}
$$

with $\xi \in \mathscr{H}$ and

$$
\begin{aligned}
\psi_{N}(\xi) & = \begin{cases}\left(|\xi|^{2}-L^{-2}\right)^{-1} & \text { if } \mid \xi=L^{-k}, k<N \\
\psi_{N}^{(T)} & \text { if }|\xi| \leqq L^{-N}\end{cases} \\
\psi_{N}^{(T)} & \equiv L^{4 N}\left(1-L^{-4}\right) \sum_{k=N}^{\infty} \frac{L^{-6 k}}{1-L^{-2(k+1)}} \\
& =\kappa \int_{|x|>L^{N}} d x q(x),
\end{aligned}
$$


Proof. Because the metric on the hierarchical lattice is an ultra-metric, the process is killed the first time it jumps a distance greater than $|\Lambda|=L^{N}$. We rewrite the expectation, conditioning on the number of steps $n$, which is a Poisson distributed random variable with mean $r t . X_{i}$ are the steps, which are iid random variables distributed according to $q(x)$. So

$$
\begin{aligned}
E_{0, N}\left(\left\langle\omega_{N}(t), \xi\right\rangle\right) & =\sum_{n=0}^{\infty} \frac{(r t)^{n}}{n !} e^{-r t} E_{0, N}\left\langle\xi, \sum_{i=1}^{n} X_{i} \mathbf{1}_{X_{i} \leqq L^{N}}\right\rangle \\
& =\exp \left(r t \int_{|x| \leqq L^{N}} d x q(x)\langle\xi, x\rangle-r t\right) \\
& \equiv e^{-t \psi(\xi)} .
\end{aligned}
$$

The calculation of $\psi(\xi)$ is an application of the fourier inversion formula

$$
\hat{\mathbf{1}}_{\mathscr{C}_{k}}=L^{4 k} \mathbf{1}_{\mathscr{H}_{k}},
$$

which is $[\mathrm{BEI}]$, Lemma 2.1. We insert the definition fo $q(x)$ and find

$$
\begin{aligned}
& \int_{|x| \leqq L^{N}} d x q(x)\langle\xi, x\rangle=\int d x\langle\xi, x\rangle\left[\mathbf{1}_{|x|=0}\right. \\
& -\sum_{k=0}^{N-1} \frac{L^{-6 k}}{\left(1-L^{-2(k+1)}\right)}\left(\mathbf{1}_{|x| \leqq L^{k}}-L^{-4} \mathbf{1}_{|x| \leqq L^{k+1}}\right) \\
& \left.-\left(1-L^{-4}\right) \sum_{k=N}^{\infty} \frac{L^{-6 k}}{\kappa\left(1-L^{-2(k+1)}\right)} \mathbf{1}_{|x| \leqq L^{N}}\right] \\
& =1-\sum_{k=0}^{N-1} \kappa^{-1}\left(L^{2 k}-L^{-2}\right)^{-1} \mathbf{1}_{|\xi|}=L^{-k} \\
& -\mathbf{1}_{|\xi|} \leqq L^{-N} L^{4 N}\left(1-L^{-4}\right) \sum_{k=N}^{\infty} \frac{L^{-6 k}}{\kappa\left(1-L^{-2(k+1)}\right)},
\end{aligned}
$$

which immediately yields the result.

Next we calculate the Green's function for the killed process and find a decomposition similar to (2.2).

Lemma 2.3. For any $x, y \in \Lambda$,

where

$$
G_{N}(x, y)=\sum_{k=0}^{N-1} L^{-2 k} \Gamma\left(x / L^{k}\right)+F_{N},
$$

$$
\begin{aligned}
\Gamma(x) & =\left(1-L^{-2}\right) \mathbf{1}_{|x|=0}, \\
F_{N} & =L^{-2(N+1)}\left(1-L^{-2 N}\right)+L^{-4 N}\left(\psi_{N}^{(T)}\right)^{-1} .
\end{aligned}
$$

When $N=0$, omit the sum in (2.3).

Proof.

$$
\begin{aligned}
\hat{G}_{N}(\xi) & =\psi(\xi)^{-1} \\
& =\sum_{k=0}^{N-1}\left(L^{2 k}-L^{-2}\right) \mathbf{1}_{|\xi|=L^{-k}}+\left(\psi_{N}^{(T)}\right)^{-1} \mathbf{1}_{|\xi| \leqq L^{-N}} .
\end{aligned}
$$

Then invert the Fourier transform using (2.2). 
We now describe how to write the expectations for self-avoiding walks in terms of Berezin integrals. Given a path $\omega(t), 0 \leqq t \leqq T$, for the process, the local time spent at a site $x$ up to time $T$ is

$$
\tau_{x}^{T}=\int_{0}^{T} d s \mathbf{1}_{\{\omega(s)=x\}} .
$$

Since our walks are killed on first exit from the volume $\Lambda=\mathscr{G}_{N}$, we have that

$$
\tau(\Lambda)=\int_{\Lambda} d x \tau_{x}^{T}=T
$$

A measure of self-interactions at a point $x$ for a walk living for time $T$ is

$$
\frac{1}{2}\left(\tau_{x}^{T}\right)^{2}=\int_{0 \leqq s_{1} \leqq s_{2} \leqq T} d s_{1} d s_{2} \mathbf{1}_{\left\{\omega\left(s_{1}\right)=\omega\left(s_{2}\right)=x\right\}},
$$

and so to measure the self-interactions of the entire walk define

$$
\tau^{2}(\Lambda) \equiv \int_{\Lambda} d x\left(\tau_{x}^{T}\right)^{2}
$$

We are conditioning on walks beginning and ending at the origin, and hence define the expectation

$$
\begin{aligned}
E_{a, \lambda, N}(F((\vec{\tau})) & \equiv \frac{1}{N^{\prime}} \int_{0}^{\infty} d t e^{-a t} E_{0, N}\left(e^{-\lambda \tau^{2}\left(\mathscr{G}_{N}\right)} F\left(\vec{\tau}^{t}\right) \mathbf{1}_{\{\omega(t)=0\}} \mid \omega(0)=0\right) \\
& =\frac{1}{N^{\prime}} \int_{0}^{\infty} d t E_{0, N}\left(e^{-a \tau^{2}\left(\mathscr{G}_{N}\right)-\lambda \tau^{2}\left(\mathscr{G}_{N}\right)} F\left(\vec{\tau}^{t}\right) \mathbf{1}_{\{\omega(t)=0\}} \mid \omega(0)=0\right)
\end{aligned}
$$

where $\vec{\tau}^{t}=\left(\tau_{1}^{t}, \ldots, \tau_{n}^{t}\right), N^{\prime}$ is defined so that $E_{a, \lambda, N}(1)=1$, and we have used (2.4).

\section{Lemma 2.4.}

$$
E_{a, \lambda, N}(F(\vec{\tau}))=\frac{S\left(F\left(\boldsymbol{\Phi}^{2}\right) e^{-\int_{\mathscr{G}_{N}} d x v\left(\Phi_{x}\right)}\right)}{S\left(e^{-\int_{G_{N}} d x v(\Phi)}\right)}
$$

where

$$
\begin{aligned}
v(\boldsymbol{\Phi}) & \equiv a \boldsymbol{\Phi}^{2}+\lambda \boldsymbol{\Phi}^{4}, \\
S(\cdot) & \equiv \int d \mu_{G_{N}}(\boldsymbol{\Phi})(\cdot) \bar{\varphi}_{0} \varphi_{0} .
\end{aligned}
$$

Proof. Apply [BEI], Theorem 3.3, to (2.5).

\section{Renormalization Group}

We will now define the renormalization group map, which will be used to calculate the functions $S(\cdot)$ appearing in Lemma 2.4. Techniques for handling Gaussian integrals with combined Fermionic-Bosonic measures can be found in [BEI] and references therein. Also in [BEI], Sect. 4, is a discussion of the renormalization group framework we use, though the details here are slightly different. For this reason we will briefly sketch the construction of the RG map. 
The functions $S(\cdot)$ are of the form $\int d \mu_{G_{N}} f^{\Lambda}$, with $f^{\Lambda} \equiv \prod_{z \in \Lambda} f_{z}\left(\Phi_{z}\right)$. Suppose $a_{i}, i=0, \ldots, N$ are in $\mathbb{R}$; we will define what values they take below. When we write $G_{N}+a_{i}$ as a covariance matrix $C$ we mean $C_{x, y}=G_{N}(x-y)+a_{i}$. Define

$$
G^{\prime}(x)=L^{-2} G_{N-1}(x / L)+\delta F_{N}+a_{N},
$$

where $\delta F_{N} \equiv F_{N}-L^{-2} F_{N-1}$. Then, referring to Lemma 2.3, we have that

$$
\int d \mu_{G_{N}+a_{N}}(\Phi) f^{\Lambda}(\Phi)=\int d \mu_{G^{\prime}}\left(\Phi^{\prime}\right) d \mu_{\Gamma}(\zeta) f^{\Lambda}\left(\Phi^{\prime}+\zeta\right) .
$$

The covariance $G^{\prime}$ is constant on blocks $x+\mathscr{G}_{1}$, which means that in the integrand $\Phi_{x}^{\prime}=\Phi_{y}^{\prime}$ almost surely $d \mu_{G^{\prime}}$ if $x-y \in \mathscr{G}_{1}$. This allows us to eliminate all but one field per block in the integrand; we call these block fields $\Phi_{L z}, z \in \Lambda / L$. We next rescale, while involves the change of variables $\Phi_{L z} \rightarrow L^{-1} \Phi_{x}$, and results in

$$
\int d \mu_{G_{N}+a_{N}}(\Phi) f^{\Lambda}(\Phi)=\int d \mu_{G_{N-1}+a_{N-1}}(\Phi)(T f)^{\Lambda / L}(\Phi),
$$

where $a_{N-1} \equiv L^{2} \delta F_{N}+L^{2} a_{N}$, and we define the reormalization group transformation (RGT) by

$$
\begin{aligned}
(T f)_{z} & \equiv \mathscr{R} \mu_{\Gamma} * f^{z+\mathscr{G}_{1}} \\
& =\int d \mu_{\Gamma}(\zeta) \prod_{u \in z+\mathscr{G}_{1}} f_{u}\left(\frac{\Phi_{z}}{L}+\zeta_{u}\right)
\end{aligned}
$$

for $z \in \Lambda / L$. The rescaling operator $\mathscr{R}$ is an algebra homomorphism defined by its action on the generators and the coefficient ring $C^{\infty}\left(\mathbb{R}^{2 N}\right)$ :

$$
\begin{aligned}
\mathscr{R}\left(\psi_{z}^{\#}\right) & \equiv L^{-1} \psi_{x}^{\#}, \quad \forall z \in L x+\mathscr{G}_{1}, \quad \psi^{\#}=\psi \text { or } \bar{\psi}, \\
\mathscr{R}\left(\varphi_{z}\right) & \equiv L^{-1} \varphi_{x}, \quad \forall z \in L x+\mathscr{G}_{1}, \\
(\mathscr{R} f)(\varphi) & \equiv f(\mathscr{R} \varphi), \quad f \in C^{\infty}\left(\mathbb{R}^{2 N}\right) .
\end{aligned}
$$

We can now read off how we should define all of the $a_{i}$ for our application. We have $a_{N}=0$ and, for $j=1, \ldots, N$,

$$
a_{N-j}=\sum_{i=1}^{j} L^{2 i} \delta F_{N-j+1} .
$$

Sine $\delta F_{i}=O\left(L^{-4 i}\right)$, we see that $a_{0}=O\left(L^{-2}\right)$, which will be an important fact in the sequel. We will also sometimes want to index $G$ by the induction step instead of the size of the volume, so we define

$$
G^{(n)} \equiv G_{N-n}, \quad a^{(n)} \equiv a_{N-n} .
$$

\section{Analyticity and Norms}

We use the same framework for analyticity as that used in [BEI]. We recall the definitions and main lemmas here, though we refer to that paper for proofs.

Let $\varphi=\left(\varphi_{1}, \ldots, \varphi_{p}\right) \in \mathbb{C}^{p}$. For $g(\varphi)$ a complex $C^{\infty}$ function, define

$$
\begin{aligned}
|g|_{w} & =\sup _{\varphi}|g(\varphi)| \prod_{i=1}^{p} w\left(\varphi_{i}\right)^{-1}, \\
|g|_{w, h} & =\sum_{\alpha} \frac{h^{|\alpha|}}{\alpha !}\left|g^{(\alpha)}\right|_{w} .
\end{aligned}
$$


Here $w$ is a positive weight function and $g^{(\alpha)}$ is the $\alpha^{\text {th }}$ derivative of $g$ with respect to $\varphi$ and $\bar{\varphi} . \alpha$ is a multi-index.

Next suppose $g$ belongs to a Grassmann alebra $\mathbb{G}$, so

$$
g=\sum_{\beta} g^{(\beta)}(\varphi) \psi^{\beta}
$$

This is the situation we are concerned with in this paper, so we define the following norm on such functions.

Definition 4.1.

$$
|g|_{w, h}=\sum_{\beta, \alpha} \frac{h^{\beta}}{\beta !} \frac{h^{\alpha}}{\alpha !}\left|g^{(\beta, \alpha)}\right|_{w},
$$

where $\beta, \alpha$ are multi-indexes with components of $\beta=0$ or 1 .

We will need the following facts, true for all weight functions:

Lemma 4.1 (Properties of $|\cdot|_{w, h}$ ) [BEI]. Suppose $\mu_{C}$ is a combined Fermionic and Bosonic integral, and $g \in \mathbb{G}$. Then

(i) $|g|_{w, h} \leqq|g|_{w^{\prime}, h^{\prime}}$, if $h^{\prime} \geqq h, w \geqq w^{\prime}$.

(ii) $\left|\mu_{C} * g\right|_{w^{\prime}, h} \leqq \exp \left(\sum_{i, j} h^{-1}\left|C_{i j}\right| h^{-1}\right)|g|_{w, h}$, where $w^{\prime}$ satisfies $\mu_{C} * w \leqq w^{\prime}$.

(iii) $\left|\left(\frac{\partial}{\partial \Phi}\right)^{\alpha} g\right|_{w, h} \leqq \alpha !\left(h^{\prime}-h\right)^{-\alpha}|g|_{w, h^{\prime}}$, if $h^{\prime}>h$.

We now specialize to the two different forms of $w(\varphi)$ we will be using. Let $X$ be a finite subset of the infinite lattice $\mathscr{G}$, let $\mathbb{G}_{X}$ be the Grassmann algebra generated by the fields $\Phi_{x}, x \in X$, and let $g_{X}$ denote an element in $\mathbb{G}_{X}$. In the low mass region (Sects. 5.1 and 6.1), we will take

$$
w(X, \varphi))=\exp \left(-a \int_{X} d x\left|\varphi_{x}\right|^{2}\right)
$$

and we write $|g X|_{a, h}$ to denote the norm with this weight function. We have the following properties for this norm:

Lemma 4.2. (Properties of $\left.|\cdot|_{a, h}\right)[\mathrm{BEI}]$. Suppose $h \geqq 0, g_{X} \in \mathbb{G}_{X}$. Then

(i) If $X \in \mathscr{G}_{1}$, then $\left|\mathscr{R} g_{X}\right|_{a, h}=\left|g_{X}\right| \frac{L^{2} a}{|x|}, \bar{L}$

(ii) $\left|g_{X}\right|_{a, h} \leqq\left|g_{X}\right|_{a^{\prime}, h^{\prime}}$ if $a^{\prime} \geqq a, h^{\prime} \geqq h$.

(iii) $\left|\mu_{C} * g_{X}\right|_{a, h} \leqq \exp \left[\int d x d y|C(x, y)| h^{-2}\right]\left|g_{X}\right|_{\tilde{a}, h}, \quad \tilde{a}=a(1-a\|C\|)^{-1}$, where $\|C\|$ is the norm of the covriance $C(x, y)$ regarded as an opertor on $L^{2}(X, d z)$ and $a \geqq 0$.

(iv) If $X \cap Y=\varnothing,\left|g_{X} g_{Y}\right|_{a, h} \leqq\left|g_{X}\right|_{a, h}\left|g_{Y}\right|_{a, h}$.

(v) $\left|g_{X} g_{Y}\right|_{a+b, h} \leqq\left|g_{X}\right|_{a, h}\left|g_{Y}\right|_{b, h}$.

In the higher mass region (Sects. 5.2-5.4 and 6.2-6.4), we will take

$$
w(X, \varphi)=\exp \left[\int_{X} d x\left(K-\alpha g_{2}\left(\left|\varphi_{x}\right|-A\right)^{2}\right)\right],
$$


and we write $\left|g_{X}\right|_{K, \alpha g_{2}, A, h}$. Let $n^{\prime}$ be the step at which we begin applying this form of the norm. We will have $g_{2}^{\left(n^{\prime}\right)} \sim \sqrt{\lambda}$ and, in the ensuing steps, $g_{2}^{(n)}$ will increase by a factor by approximately $L^{2}$ each step until it reaches one (see Sects. 5.3-5.4 for details on the flow), at which point its increase slows down as it approaches the fixed point of $O\left(L^{2}\right)$. For technical reasons we need to keep track of some of the decay properties of the remainder, which we measure by $\alpha g_{2}$, but we also have to give up a litle decay at each step while we are still in the regime $g_{2}<1$ (there are $O\left(-\log _{L} \lambda\right)$ such steps). We accomplish this by changing $\alpha$ at each step; i.e. we use $\alpha^{(0)}$ for the first such step, then $\alpha^{(1)}, \ldots$, and we define

$$
\alpha^{(n)}=\frac{1}{32}+\frac{1}{32} e^{-\delta n}
$$

with some $\delta>0$. Using the fact that there are $O\left(-\log _{L} \lambda\right)$ steps before $g_{2}$ reaches unity, we see that in this regime $\alpha^{(n)}-\alpha^{(n+1)} \geqq O(\delta) \lambda^{\delta / \log L}$.

Some properties of this norm are contained in

Lemma 4.3 (Properties of $|\cdot|_{K, a, A, h}$ ).

(i) If $X \subset \mathscr{G}_{1}$, then $\left|\mathscr{R} g_{X}\right|_{K, a, A, h}=\mid g_{X} \frac{K}{|X|}, \frac{L^{2} a}{|X|}, \frac{A}{L}, \frac{h}{L}$.

(ii) $\left|g_{X}(w \cdot)\right|_{K, a, A, h}=\left|g_{X}(\cdot)\right|_{K, \frac{a}{w^{2}}, A w, h w}$, for $w \in \mathbb{R}^{+}$.

(iii) $\left|g_{X}\right|_{K, a, A, h} \leqq\left|g_{X}\right|_{K, a^{\prime}, A, h^{\prime}}$ if $h^{\prime} \geqq a^{\prime} \geqq a$.

(iv) If $|\tilde{A}-A| \leqq \delta$ for some $\delta>0$, then $\left|g_{X}\right|_{K, a, A, h} \leqq O(1)\left|g_{X}\right|_{K, \tilde{a}, \tilde{A}, h}$, where $\tilde{a}=a+O\left(a^{2} \delta^{2}\right)$.

(v)

$$
\left|\mu_{t \Gamma W} * g_{X}\right|_{K, a^{(n+1)}, A, h} \leqq O(1) \exp \left(\sum_{i, j} h^{-1}\left|t \Gamma W_{i j}\right| h^{-1}\right)\left|g_{X}\right|_{K, a^{(n)}, A, h},
$$

where we have set $t \in[0,1], \Gamma W(x, y)=(\gamma w) \delta_{x, y}, \gamma w=O(1), c=O(1)$,

$$
\begin{gathered}
a^{(n+1)}=\frac{\alpha^{\prime} g_{2}^{(n+1)}}{L^{2} w^{2}}(1+c \lambda), \quad a^{(n)}=\alpha g_{2}^{(n)}, \\
g_{2}^{(n+1)}=\frac{L^{2} w^{2} g_{2}^{(n)}}{1+g_{2}^{(n)} \gamma w}+O\left(\frac{g_{2}^{(n)}}{A}\right),
\end{gathered}
$$

and

$$
\begin{gathered}
\alpha^{(n)} \geqq \alpha \geqq\left\{\begin{array}{ll}
\alpha^{(n+1)}+\frac{2}{3}\left(\alpha^{(n)}-\alpha^{(n+1)}\right) & \text { if } g_{2}^{(n)}<1 \\
\frac{5}{6} \alpha^{(n+1)} & \text { if } g_{2}^{(n)} \geqq 1
\end{array},\right. \\
\alpha^{(n+1)} \leqq \alpha^{\prime} \leqq\left\{\begin{array}{ll}
\alpha^{(n+1)}+\frac{1}{3}\left(\alpha^{(n)}-\alpha^{(n+1)}\right) & \text { if } g_{2}^{(n)}<1 \\
\frac{7}{6} \alpha^{(n+1)} & \text { if } g_{2}^{(n)} \geqq 1
\end{array} .\right.
\end{gathered}
$$

(vi) If $X \cap \varnothing,\left|g_{X} g_{Y}\right|_{K, a, A, h} \leqq\left|g_{X}\right|_{K, a, A, h}\left|g_{Y}\right|_{K, a, A, h}$.

(vii) $\left|g_{X} g_{Y}\right|_{J+K, a+b, A, h} \leqq\left|g_{X}\right|_{J, a, A, h}\left|g_{Y}\right|_{K, b, A, h}$.

The proof is contained in Appendix A. This lemma will be used in Sects 5.3, 5.4, 6.3, and 6.4. The freedom in the choices of $\alpha$ and $\alpha^{\prime}$ is not needed until Sect. 6. 
It is not very convenient to compute these norms from the definitions. We can frequently take advantage of facts we know about the analytic structure of the functions we norm. We write

$$
\varphi_{i}=\varphi_{i}^{(1)}+i \varphi_{i}^{(2)}, \quad \bar{\varphi}_{i}=\varphi_{i}^{(1)}-i \varphi_{i}^{(2)},
$$

and allow the $\varphi_{i}^{(j)}$ to be complex. We set

$$
\begin{aligned}
D_{h}(\varphi)= & \left\{\left(\varphi_{i}^{\prime}, \bar{\varphi}_{i}^{\prime}, u_{i}^{\prime}, \bar{u}_{i}^{\prime}\right) \in \mathbb{C}^{4}: \forall i=1, \ldots, p,\right. \\
& \left.\forall j=1,2,\left|\varphi_{i}^{\prime(j)}-\varphi_{i}^{(j)}\right|,\left|u_{i}^{\prime}\right|,\left|\bar{u}_{i}^{\prime}\right| \leqq h\right\}, \\
S_{h}= & \bigcup_{\varphi^{(J)} \in \mathbb{R}} D_{h}(\varphi) .
\end{aligned}
$$

If we have a function $F$ analytic on $D_{h}(\varphi)$, define

$$
|F|_{h}(\varphi)=\sup _{\varphi^{\prime} \in D_{h}(\varphi)}\left|F\left(\varphi^{\prime}\right)\right|,
$$

and let $F(\Phi)$ denote an element of the Grassmann algebra obtained by substituting $u=\psi, \bar{u}=\bar{\psi}$ in the power series

$$
F(\varphi, \bar{\varphi}, \bar{u}, \bar{u})=\sum \frac{1}{\bar{\alpha} ! \alpha !}\left(\partial_{\bar{u}, u}^{\bar{\alpha}, \alpha} F\right)(\bar{\varphi}, \varphi, 0,0) \bar{u}^{\bar{\alpha}} u^{\alpha},
$$

with any convention for the order of $\psi$ 's in the product $\left(\alpha !=\alpha^{\prime} !=1\right)$.

Lemma 4.4 (Comparison of Norms) [BEI]. Suppose $F$ is analytic in $S_{c h}$ and $g=F(\Phi)$, then for $c>1$,

where

$$
|g|_{w, h} \leqq\left(\frac{1}{1-c^{-1}}\right)^{4 p}\|F\|_{w, c h},
$$

$$
\|F\|_{w, h} \equiv \sup _{\varphi \in S_{h}}|F|_{h}(\varphi) w^{-1}(\varphi) .
$$

We will also use a corollary of this lemma that is specific to functions in $\mathbb{G}$ that are of the form $g=G\left(\Phi^{2}\right)$, where $G$ is a function of a single variable. In order to apply the above lemma, we need to bound $G\left(\varphi^{\prime} \bar{\varphi}^{\prime}+u^{\prime} \bar{u}^{\prime}\right) w(\varphi)^{-1}$ for $\varphi \in \mathbb{C}$, $\left(\varphi^{\prime}, \varphi^{\prime}, u^{\prime}, \bar{u}^{\prime}\right) \in D_{c h}(\varphi)$. To make matters simpler, we could just bound $G(v) w(\varphi)^{-1}$ for $\varphi \in \mathbb{C}, v \in D_{c h}^{(1)}(\varphi)$, where

$$
\begin{aligned}
D_{c h}^{(1)}(\varphi) \equiv & \left\{z \in \mathbb{C}: z=\varphi^{\prime} \bar{\varphi}^{\prime}+u^{\prime} \bar{u}^{\prime}\right. \\
& \text { for some } \left.\left(\varphi^{\prime}, \bar{\varphi}^{\prime}, u^{\prime}, \bar{u}^{\prime}\right) \in D_{c h}(\varphi)\right\}
\end{aligned}
$$

or, simpler still, we define $C_{c h}(\varphi)$ to be a disc around $|\varphi|^{2}$ of radius $r_{c h}(\varphi)$ chosen so that $D_{c h}^{(1)}(\varphi) \subset C_{c h}(\varphi)$. We set

and also define

$$
r_{h}(\varphi)= \begin{cases}8 h^{2} & \text { if }|\varphi| \leqq h \\ 8 h|\varphi| & \text { if }|\varphi|>h\end{cases}
$$

We now have the following

$$
R_{h}=\bigcup_{\varphi \in \mathbb{C}} C_{h}(\varphi) .
$$


Corollary 4.5. If $G$ is a function of a single complex variable, analytic in $R_{c h}$, $g=G\left(\Phi^{2}\right)$, then

$$
|g|_{w, h} \leqq\left(\frac{1}{1-c^{-1}}\right)^{4} \sup _{\substack{\varphi \in \mathbb{C} \\ l \in C_{c h}(\varphi)}} \mid G\left(|\varphi|^{2}+l\right) w(\varphi)^{-1}
$$

At times we will want pointwise bounds on a function given bounds on its norm. For this we have

Lemma 4.6. Suppose $F$ is a function of a single variable $z$, and $G(\Phi) \equiv \Phi^{2 n} F\left(\Phi^{2}\right)$ satisfies $|G|_{w, h}<\infty$. Set $z=\varphi^{\prime} \bar{\varphi}^{\prime}+l^{\prime}$, with $\varphi^{\prime}, \bar{\varphi}^{\prime}$ complex conjugates, $l^{\prime} \in \mathbb{C}$ with $\left|l^{\prime}\right|<\max \left(\left(\frac{h}{2}\right)^{2}, \frac{h}{2}|\varphi|\right)$. Then

$$
\left|F^{(\alpha)}\left(\varphi^{\prime} \bar{\varphi}^{\prime}+l^{\prime}\right)\right| \leqq\left\{\begin{array}{ll}
O(1) h^{-2(n+\alpha)}|G|_{w, h} w(0) & \text { if }\left|\varphi^{\prime}\right| \leqq 3 h / 4 \\
O(1)\left(h\left|\varphi^{\prime}\right|\right)^{-\alpha}\left|\varphi^{\prime}\right|^{-2 n}|G|_{w, h} w\left(\varphi^{\prime}\right) & \text { if }\left|\varphi^{\prime}\right|>3 h / 4
\end{array}\right. \text {. }
$$

Proof. Let $f\left(\varphi^{\prime}, \bar{\varphi}^{\prime}\right) \equiv\left(\varphi^{\prime} \bar{\varphi}^{\prime}\right)^{n} F\left(\varphi^{\prime} \bar{\varphi}^{\prime}\right)$. Clearly $|f|_{w, h} \leqq|G|_{w, h}$, and so $f$ is analytic in $\varphi^{\prime}, \bar{\varphi}^{\prime}$ with radius of convergence $h$. We use this fact in two different ways depending on the size of $\left|\varphi^{\prime}\right|$.

(i) If $\left|\varphi^{\prime}\right| \leqq 3 h / 4$, then $\left|\varphi^{\prime} \bar{\varphi}^{\prime}+l^{\prime}\right|<15 h^{2} / 16$. So we choose some $w, \bar{w}$ not necessarily complex conjugates such that $w \bar{w}=\varphi^{\prime} \bar{\varphi}^{\prime}+l^{\prime}$ with $|w|,|\bar{w}|<h$. Then we expand

$$
f(w, \bar{w})=\left.\sum_{\beta, \bar{\beta}} \frac{w^{\beta} \bar{w}^{\bar{\beta}}}{\beta ! \bar{\beta} !} \frac{\partial^{\beta}}{\partial \varphi^{\beta}} \frac{\partial^{\bar{\beta}}}{\partial \bar{\varphi}^{\bar{\beta}}} f\right|_{\varphi=\bar{\varphi}=0} .
$$

Now,

$$
F^{(\alpha)}(w \bar{w})=\bar{w}^{-\alpha} \frac{\partial^{\alpha}}{\partial w}\left[(w \bar{w})^{-n} f(w, \bar{w})\right]
$$

which tells us that

$$
F^{(\alpha)}(w \bar{w})=\left.\sum_{\beta, \bar{\beta}}(\beta-n) \cdots(\beta-n-\alpha+1) \frac{w^{\beta-n-\alpha} \bar{w}^{\bar{\beta}-n-\alpha}}{\beta ! \bar{\beta} !} \frac{\partial^{\beta}}{\partial \varphi^{\beta}} \frac{\partial^{\beta \bar{\beta}}}{\partial \bar{\varphi}^{\bar{\beta}}} f\right|_{\varphi=\bar{\varphi}=0} .
$$

Now, because $f\left(\varphi^{\prime}, \bar{\varphi}^{\prime}\right)$ is a function of $\varphi^{\prime} \bar{\varphi}^{\prime}$ alone, and analytic at the origin, we have that $\partial_{\varphi^{\prime}}^{\beta},\left.\partial_{\bar{\varphi}^{\prime}}^{\bar{\beta}} f\right|_{\varphi=\bar{\varphi}=0}=0$ unless $\beta=\bar{\beta}$. Note that $F$ is regular at the origin, for otherwise $|G|_{w, h}$ would be infinity due to the $F^{\prime}$ term in $F\left(\Phi^{2}\right)=F(\varphi \bar{\varphi})+\psi \bar{\psi} F^{\prime}(\varphi \bar{\varphi})$. Hence $\left.\partial_{\varphi}^{i} \partial_{\bar{\varphi}}^{i} f\right|_{\varphi=\bar{\varphi}=0}$ for $i=0, \ldots, n-1$. Also, we can use Lemma 4.1 to show that

$$
\left.\frac{\partial^{\beta}}{\partial \varphi^{\beta}} \frac{\partial^{\beta}}{\partial \bar{\varphi}^{\beta}} f\right|_{\varphi=\bar{\varphi}=0} \leqq(\beta !)^{2} h^{-2 \beta}|f|_{w, h} w(0) .
$$

Inserting this in (4.5), we find that

$$
\left|F^{(\alpha)}\left(\varphi^{\prime} \bar{\varphi}^{\prime}+l^{\prime}\right)\right| \leqq O(1) h^{-2(n+\alpha)}|f|_{w, h} w(0) .
$$


(ii) If $\left|\varphi^{\prime}\right|>3 h / 4$, we have $F\left(\varphi^{\prime} \bar{\varphi}^{\prime}+l^{\prime}\right)=(w \bar{w})^{-n} f(w, \bar{w})$ with $w=\varphi^{\prime}+l^{\prime} / \bar{\varphi}^{\prime}$ and $\bar{w}=\bar{\varphi}^{\prime}$. We will apply (4.4) again, this time expanding $f$ around $\varphi^{\prime}$ and $\bar{\varphi}^{\prime}$. For any $m$,

$$
\frac{\partial^{m}}{\partial w^{m}} f(w, \bar{w})=\left.\sum_{\beta, \bar{\beta}} \beta \cdots(\beta-m+1) \frac{\left(w-\varphi^{\prime}\right)^{\beta-m}\left(\bar{w}-\bar{\varphi}^{\prime}\right)^{\bar{\beta}}}{\beta ! \bar{\beta} !} \frac{\partial^{\beta}}{\partial \varphi^{\beta}} \frac{\partial^{\bar{\beta}}}{\partial \bar{\varphi}^{\bar{\beta}}} f\right|_{\substack{\varphi=\varphi^{\prime} \\ \bar{\varphi}=\bar{\varphi}^{\prime}}} .
$$

Next use Lemma 4.1 to get the bounds

$$
\left.\frac{\partial^{\beta}}{\partial \varphi^{\beta}} \frac{\partial^{\bar{\beta}}}{\partial \bar{\varphi}^{\bar{\beta}}} f\right|_{\substack{\varphi=\varphi^{\prime} \\ \bar{\varphi}=\bar{\varphi}^{\prime}}} \leqq \beta ! \bar{\beta} ! h^{-(\beta+\bar{\beta})}|f|_{w, h} w\left(\varphi^{\prime}\right) .
$$

Inserting this in (4.6) and noting that with this choice of $w, \bar{w}$ the only contribution from the sum over $\bar{\beta}$ is the $\bar{\beta}=0$ term, we find

$$
\left|\frac{\partial^{m}}{\partial w^{m}} f(w, \bar{w})\right| \leqq O(1) h^{-m}|f|_{w, h} w\left(\varphi^{\prime}\right) .
$$

Finally notice that, again for any $m$,

$$
\left|\frac{\partial^{m}}{\partial w^{m}}(w, \bar{w})^{-n}\right| \leqq c(n)\left|\varphi^{\prime}\right|^{-2 n-m}
$$

Inserting (4.8) and (4.7) into (4.4) completes the proof.

Corollary 4.7. If the weight function $w$ is of the form (4.1), with $g_{2}=4 \lambda A^{2} \geqq$ $L^{-2} \sqrt{\lambda}, h=\min \left(\lambda^{-1 / 4}, g_{2}^{-1 / 2}\right)$, and $F, G, \varphi^{\prime}, \bar{\varphi}^{\prime}, l^{\prime}$ are as in Lemma 4.6, then

$$
\left.\left|F^{(\alpha)}\left(\varphi^{\prime} \bar{\varphi}^{\prime}+l^{\prime}\right) \leqq O\left(\lambda^{\alpha / 2}\right) A^{-2 n}\right| G\right|_{K, \alpha g_{2}, A, h} e^{K-\frac{1}{2} \alpha g_{2}\left(\left|\varphi^{\prime}\right|-A\right)^{2}} .
$$

The hypotheses of Corollary 4.7 are such that it will be applicable in Sects. $5.2-5.4$ and $6.2-6.4$.

\section{Renormalization of the Interaction}

In order to compute the expectation of the density at site $z$, we must compute the functional integrals $S\left(I_{\Lambda}\right)$ and $S\left(\Phi_{z}^{2} I_{\Lambda}\right)$, where the interaction $I_{\Lambda}$ is defined to be

$$
\begin{gathered}
I_{\Lambda} \equiv \prod_{x \in \Lambda} g\left(\Phi_{x}\right), \\
g(\Phi)=\exp (-v(\Phi)), \\
v(\Phi)=\lambda \Phi^{4}+a \Phi^{2} .
\end{gathered}
$$

We begin by computing the RGT in those blocks that do not contain 0 or $z$, so the RGT applies to the function $g^{\mathscr{G}_{1}}$, where

$$
g^{X}=\prod_{x \in X} g\left(\Phi_{x}\right)
$$

This proceeds by three different methods depending on the size of the mass. 
5.1. Small Mass Flow. The existence of a critical point follows from Proposition 5.1, below. We wish to study our model at masses below the critical one, and follow the approach to the critical point carefully. For masses very close to the critical one, a slight perturbation of the methods of [BEI] apply. These were developed in [BI], and for completeness we present them briefly here. After zero or more RGT's, the function $T^{n} g$ can be represented as

Inductive Assumption $1(\beta, \lambda, M)$. The function $g(\Phi)$ can be represented as

$$
\begin{aligned}
e^{\beta \Phi^{2}} g(\Phi) & =e^{\beta \Phi^{2}}\left(e^{-v}\left(1+\eta: \Phi^{6}:_{G_{M}+a_{M}}\right)+r(\Phi)\right), \\
v(\Phi) & =\lambda: \Phi^{4}:_{G_{M}+a_{M}}+\mu_{c}^{2}: \Phi^{2}:_{G_{M}+a_{M}} .
\end{aligned}
$$

Here $\mu_{c}^{2}=a_{c}(\lambda)+2 \lambda G_{\infty}(0)$, the parameter $\eta$ satisfies $\eta<c_{0} \lambda^{2}$, and $r(\Phi)=\mathrm{R}\left(\Phi^{2}\right)$ satisfies

$$
\begin{aligned}
|r| \sqrt{\lambda}, h & \leqq c_{1} \lambda ; \quad h=\lambda^{-1 / 4}, \\
\left.\frac{d^{j}}{d t^{j}} r(t \Phi)\right|_{t=0} & =0, \quad 0 \leqq j<8 .
\end{aligned}
$$

Proposition 5.1 ([BEI] Theorem 7.2). Choose some integer L sufficiently large, and then some $\lambda_{0}$ sufficiently small. Then there exists $a_{c}\left(\lambda_{0}\right)=-2 \lambda_{0} G_{\infty}(0)+O\left(\lambda_{0}^{2}\right)$, such that if

$$
g(\Phi)=\exp \left(-\lambda_{0} \Phi^{4}-a_{c} \Phi^{2}\right)
$$

then $T^{n} g$ satisfies $I A 1(0, \lambda, \infty)$ with $\lambda_{n}=\left(\lambda_{1}^{-1}+\beta_{2} n+O(\log n)\right)^{-1}$ for all $n$. Here $O(\cdot)$ may depend on $L$.

Proof. Our model is slightly different than that used in [BEI], but this is irrelevant since they did not take advantage of the special feature of theirs that the fluctuation covariance was singular. This is borne out by the analysis below: take $\beta=0$ in our Proposition 5.2, and substitute this for [BEI] Proposition 7.1 in the proof of [BEI] Proposition 7.2.

We are interested in the case of $\beta>0$ small, so we choose $0<\beta<\lambda^{1 / 2}$. Before the first step, our starting $g$ satisfies $I A 1(\beta, \lambda, N)$ with $r=\eta=0$.

At the end of the induction we will see that our covariance $G_{N}$ is not quite massless; it has a mass that vanishes exponentially as the volume tends to $\infty$. Because the $a_{c}(\lambda)$ we are using in $I A 1$ is defined by the infinite-volume process, we must correct for this by adding a small negative mass to $\beta$; i.e. to calculate the expectation of the density in finite volume with negative killing rate $\beta$, we must start with the interaction function $\exp \left(\left(\beta+\beta_{\Lambda}\right) \Phi^{2}\right) g\left(\Phi^{2}\right)$. For simplicity of notation, we will set $\beta^{\prime}=\beta+\beta_{\Lambda}$ and then drop the prime.

We apply the RGT:

$$
T\left(e^{\beta \Phi^{2}} g\right)=\mathscr{R}_{\mu \Gamma} *\left(e^{\beta \Phi^{2}} g\right)^{\mathscr{G}_{1}} .
$$

If there were no interactions, we could do the convolution exactly via a translation of the superfield. We do this translation even in the presence of the interaction

$$
\zeta \rightarrow \zeta-\beta\left(\Gamma^{-1}+\beta\right)^{-1} \Phi
$$


and then

$$
\begin{aligned}
T\left(e^{\beta \Phi^{2}} g\right) & =\mathscr{R} e^{\beta \int_{\mathscr{c}_{1}} d x d y \Phi_{x} W_{x y} \bar{\Phi}_{y}} \mu_{\Gamma W} * g^{\mathscr{G}_{1}}(W \Phi) \\
W & =(1-\beta \Gamma)^{-1}
\end{aligned}
$$

or, inserting the forms for $\Gamma, W$ we have for our model,

$$
T\left(e^{\beta \Phi^{2}} g\right)=e^{\frac{L^{2} \beta}{1-\beta \gamma} \Phi^{2}} \mathscr{R} \mu_{\Gamma W} * g^{\mathscr{S}_{1}}(W \Phi)
$$

with $\gamma \equiv 1-L^{-2}$. So, keeping in mind that $\beta \rightarrow L^{2} \beta /(1-\beta \gamma)$, we can study the recursion for $g(\Phi)$ exactly as was done in [BEI], with the changes of replacing $\Gamma \rightarrow \Gamma W$ in the convolution and evaluating at $W \Phi$. Since the differences are minor, we will only briefly sketch the argument here, replacing one part of it with a more elegant method due to [BI].

Proposition 5.2. Let $L$ be large enough, and $c_{0}=c_{0}(L), c_{1}=c_{1}(L)$ in $I A 1(\beta, \lambda, M)$ be large enough. Let $0<\lambda<\lambda_{0}$, with $\lambda_{0}=\lambda_{0}(L)$ small enough. Then $e^{\beta \Phi^{2}} g(\Phi)$ satisfies $I A 1(\beta, \gamma, M)$ implies $T\left(e^{\beta \Phi^{2}} g(\Phi)\right)$ satisfies $I A 1\left(\beta^{\prime}, \lambda^{\prime}, M-1\right)$, where

$$
\begin{aligned}
& \beta^{\prime}=\frac{L^{2} \beta}{1-\beta \gamma}-\gamma_{\beta} L^{2} \beta \lambda+O\left(\beta^{2}, \beta \lambda^{2}\right), \\
& \lambda^{\prime}=\lambda-\beta_{2} \lambda^{2}+O\left(\beta \lambda, \lambda^{3}\right), \\
& \eta^{\prime}=L^{-2} \eta+O\left(\lambda^{2}\right) .
\end{aligned}
$$

and $\beta_{2}=16 \gamma\left(1-\frac{\gamma}{2}\right), \gamma_{\beta}=4 \gamma\left(1-\frac{\gamma}{2}\right)$.

Proof. We will assume $.9 \lambda \leqq \lambda^{\prime} \leqq \lambda$, which will be justified in the course of the proof. We say a term satisfies $\tilde{r}$ bounds if

$$
\begin{aligned}
|\tilde{r}|_{\sqrt{\lambda}, h} & \leqq O\left(L^{-4}\right) c_{1} \lambda, \\
\left|\tilde{r}^{(\alpha)}(0)\right| & \leqq c^{(\alpha)}(L) \lambda^{3} \quad \text { for }|\alpha|<8 .
\end{aligned}
$$

$\tilde{r}$ will be used to denote terms that satisfy $\tilde{r}$ bounds, and its value may change from line to line or even within the same line.

The $g^{\mathscr{G}_{1}}$ splits into three sets of terms: those with $\geqq 2$ remainders $r$, which we denote $S_{\geq 2}$, those with one remainder $S_{1}$, and those with no remainders $S_{0}$. Both terms with remainders are handled exactly as in [BEI]; they are $\tilde{r}$. We omit the detailed arguments, but the heuristics are as follows: the $S_{\geqq 2}$ terms are $O\left(\lambda^{2}\right)$ in norm due to the two remainders, and the derivative condition follows when we use the fact that $r(\Phi)$ starts at $O\left(\Phi^{8}\right)$. This same fact gives us the derivative conditon for the $S_{1}$ terms, and to get the norm conditon we observe that under rescaling $r$ scales down by $L^{-8}$, which compensates for the fact that there are $L^{4}$ such terms.

The remaining term is handled by a new method due to [BI]. Define

$$
\begin{aligned}
Z(t) & =\mathscr{R} \mu_{(1-t) \Gamma W} * \underline{e}^{-u(t)+\int_{0}^{t} d s \mu_{(t-s) \Gamma W^{*}} u_{\Phi}^{2}(s)}, \\
u(t) & =\mu t \Gamma W * \int_{\mathscr{G}_{1}} d x\left\{v\left(\Phi_{x}\right)-\eta: \Phi_{x}^{6}:\right\}, \\
u_{\Phi}^{2}(t) & =\int_{\mathscr{G}_{1}} d x d y(\Gamma W)(x, y) \frac{\partial u(t)}{\partial \bar{\Phi}_{x}} \frac{\partial u(t)}{\partial \Phi_{y}},
\end{aligned}
$$


and we define $e$ on Wick-ordered polynomials to be the ordinary exponential, but terms of order : $\Phi^{6}$ : are expanded out to first order, and terms of order : $\Phi^{8}:$ and

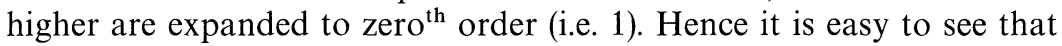

$$
Z(0)=\mathscr{R} \mu_{\Gamma W} * S_{0},
$$

which is exactly the quantity we wish to calculate, and that

$$
\frac{d}{d t} Z(t)=\tilde{r} .
$$

So, by the fundamental theorem of calculus, we have that

$$
\mathscr{R} \mu_{\Gamma W} * S_{0}=\mathscr{R} \underline{e}^{-u(t)+\int_{0}^{t} d s \mu_{(1-s) \Gamma W} u_{\Phi}^{2}(s)}+\tilde{r} .
$$

It remains to do the calculation. The leading behavior is in the $u(1)$ term. In order to calculate this, we first state the identity

$$
\left(\mathscr{R} \mu_{\Gamma W} *: \Phi^{n}:_{G_{M}+a_{M}}\right)(W \Phi)=\left(\frac{w}{L}\right)^{n} e^{B \Delta}: \Phi^{n}:_{G_{M-1}+a_{M-1}},
$$

where

$$
B=2 L^{2} \beta \gamma\left(1-\frac{\gamma}{2}-\frac{\beta \gamma}{2}\right)+\left(G_{M-1}+a_{M-1}-1\right)\left(2 \beta \gamma-\beta^{2} \gamma^{2}\right) .
$$

In particular,

$$
\left(\mathscr{R} \mu_{\Gamma W} *: \Phi^{4}:_{G_{M}+a_{M}}\right)(W \Phi)=\left(\frac{w}{L}\right)^{n}: \Phi^{4}:_{G_{M-1}+a_{M-1}}+2 B\left(\frac{w}{L}\right)^{4} \Phi^{2} .
$$

The identity follows from the definitions of $G_{M}, a_{M}, W$, and $\Gamma$, along with properties of Wick ordering, which are summarized in [BEI] Lemma 5.1. Note that $G_{M-1}+a_{M-1}-1=O\left(L^{-2 M}\right)$, and $M$ can be as large as we like since since we are interested in the infinite-volume limit and we only apply this proposition in the small-mass regime.

We can now find the leading behavior of $\lambda^{\prime}$ and $\beta^{\prime}$ from

$$
T\left(e^{\beta \Phi^{2}} g\right)=e^{\frac{L^{2} \beta}{1-\beta \gamma} \Phi^{2}}\left[\mathscr{R}\left(\underline{e}^{-u(1)+\int_{0}^{1} d s \mu_{(1-s) \Gamma W^{*}} u_{\Phi}^{2}(s)}\right)(W \Phi)+\tilde{r}\right] .
$$

The $\mu_{c}^{2}$ and $\eta$ terms in $u(1)$ do not contribute to $\lambda^{\prime}$ or $\beta^{\prime}$ at leading or next to leading order (the largest contributions are $O\left(\beta \lambda^{2}\right)$ ). So the only important terms in $u(1)$ are

$$
\mathscr{R} u(1)(W \Phi)=\lambda: \Phi^{4}: G_{M-1}+a_{M-1}+4 L^{2} \beta \gamma\left(1-\frac{\gamma}{2}\right) \lambda \Phi^{2}+\cdots .
$$

Combining this with the $L^{2} \beta \Phi^{2} /(1-\beta \gamma)$ in front, we find

$$
\begin{aligned}
& \lambda^{\prime}=\lambda+O\left(\beta \lambda, \lambda^{2}\right), \\
& \beta^{\prime}=L^{2} \beta\left(1-4 \gamma\left(1-\frac{\gamma}{2}\right) \lambda\right)+O\left(\beta^{2}, \beta \lambda^{2}\right) .
\end{aligned}
$$

Hence we have found that $\gamma_{\beta}=4 \gamma\left(1-\frac{\gamma}{2}\right)$, as stated. To see that $\beta_{2}=16 \gamma\left(1-\frac{\gamma}{2}\right)$ requires examining the $u_{\Phi}^{2}$ correction. The calculation is straightfoward, but since it 
is done in $[\mathrm{BI}]$ we will not repeat it here. We note that the ratio $\gamma_{\beta} / \beta_{2}=1 / 4$ is the same here as it was in [B1], as would be expected.

The final step is to absord the $\tilde{r}$ terms into a shift in $\beta, \lambda$, and $\eta$, which occurs exactly as in [BEI] and incurs only $O\left(\lambda^{3}\right)$ shifts in $\beta^{\prime}, \lambda^{\prime}$, and $\eta^{\prime}$.

5.2. Switch of Representations. After applying the small-mass recursion up to the point where one more RGT would result in $|\beta| \geqq \sqrt{\lambda} / 4$, we must stop using the [BI] method and use a new approach for a while before switching methods again and using the large-mass techniques. Both of the other techniques act on a different representation for the function $g$, to which we must switch. This new representation is

Inductive Assumption $2\left(\beta_{\Lambda}, K, \lambda, A, \alpha\right)$.

$$
\begin{aligned}
e^{\beta_{\Lambda} \Phi^{2}} g_{\text {new }} & =e^{\beta_{\Lambda^{\Phi^{2}}}}\left(e^{-v_{\text {new }}(\Phi)}+r_{\text {new }}\right), \\
v_{\text {new }}(\Phi) & =-K+\lambda\left(\Phi^{2}-\Lambda^{2}\right)^{2}, \\
\left|r_{\text {new }}\right|_{K, \alpha g_{2}, A, h} & \leqq c_{2} \sqrt{\lambda}, \\
\left.r_{\text {new }}^{(i)}\right|_{\Phi^{2}}=A^{2} & =0 \text { for } i<3,
\end{aligned}
$$

where $\alpha$ is described above (4.2), $h=\min \left(\lambda^{-1 / 4}, g_{2}^{-1 / 2}\right), g_{2} \equiv 4 \lambda A^{2}$ is the radial curvature of $v_{\text {new }}\left(|\varphi|^{2}\right)$ at the minimum, and, in the last line, we are considering $r=r\left(\Phi^{2}\right)$ to be a function of one variable, differentiating with respect to it $i$ times, and evaluating at $A^{2}$.

Up until now we kept most of the mass outside of the interaction (we left the critical piece $a_{c}$ inside $v$ ). At this point the mass is large enough that we must take the Mexican hat shape of the potential into account. This is reflected in $I A 2$. So we move most of the mass back into the interaction, but we still leave the volume-dependent piece $\beta_{\Lambda}$ outside. We will find that $\beta_{\Lambda}$ is very small throughout most of the induction, so it will not cause us any problems.

Proposition 5.3. $g_{\text {new }}$ satisfies $I A 2\left(\beta^{\prime}, K^{\prime}, \lambda^{\prime}, A^{\prime}, \alpha\right)$ with the choice of $c_{2}=c_{2}(L)$ large enough, and

$$
\begin{aligned}
K^{\prime} & =\left(\beta-\beta_{\Lambda}+2\left(G^{(n)}(0)+a^{(n)}\right) \lambda-\mu_{c}^{2}\right)^{2} / 4 \lambda+O(\sqrt{\lambda}), \\
A^{\prime 2} & =\left(\beta-\beta_{\Lambda}+2\left(G^{(n)}(0)+a^{(n)}\right) \lambda-\mu_{c}^{2}\right) / 2 \lambda+O(1), \\
\lambda^{\prime} & =\lambda+O\left(\lambda^{3 / 2}\right) \\
\alpha & =1 / 16 .
\end{aligned}
$$

Also,

$$
e^{\beta \Lambda^{\Phi^{2}}} g_{\text {new }}(\Phi)=e^{\beta \Phi^{2}} g(\Phi) .
$$

We will assume $\left|A^{\prime 2}-A^{2}\right| \leqq O(1)$, to be justified in the course of the proof. We begin by defining

$$
\begin{aligned}
K & =\left(\beta-\beta_{\Lambda}+2\left(G^{(n)}(0)+a^{(n)}\right) \lambda-\mu_{c}^{2}\right)^{2} / 4 \lambda \\
& =O(1) \\
A^{2} & =\left(\beta-\beta_{\Lambda}+2\left(G^{(n)}(0)+a^{(n)}\right) \lambda-\mu_{c}^{2}\right) / 2 \lambda \\
& =O\left(\lambda^{-1 / 2}\right),
\end{aligned}
$$


so that

$$
\begin{aligned}
e^{\beta \Phi^{2}} g & =e^{\beta_{\Lambda} \Phi^{2}} e^{K-\lambda\left(\Phi^{2}-A^{2}\right)^{2}}\left(1+\eta: \Phi^{6}: r\left(\Phi^{2}\right) e^{\left(\beta-\beta_{A}\right) \Phi^{2}} e^{-K+\lambda\left(\Phi^{2}-A^{2}\right)^{2}}\right) \\
& \equiv e^{\beta_{\Lambda} \Phi 2} e^{K-\lambda\left(\Phi^{2}-A^{2}\right)^{2}}\left(1+f\left(\Phi^{2}\right)\right)
\end{aligned}
$$

where the last line defines $f$. We Taylor-expand $f$ to second order around some point $B$, chosen below:

$$
f=f_{0}+f_{1}\left(\Phi^{2}-B\right)+f_{2}\left(\Phi^{2}-B\right)^{2}+f_{\geqq 3},
$$

so we can write

$$
1+f=\left(1+f_{0}\right)\left[\left(1+C\left(\Phi^{2}-B\right)+\frac{C^{2}}{2}(\Phi-B)^{2}\right)\left(1+D\left(\Phi^{2}-B\right)^{2}\right)+f_{r}\right],
$$

where

$$
\begin{aligned}
C & =\frac{f_{1}}{1+f_{0}}, \\
D & =\frac{f_{2}}{1+f_{0}}-\frac{C^{2}}{2}, \\
f_{r} & =\frac{f_{\geqq 3}}{1+f_{0}}-C D\left(\Phi^{2}-B\right)^{3}-\frac{C^{2} D}{2}\left(\Phi^{2}-B\right)^{4},
\end{aligned}
$$

which allows us to write

$$
\begin{aligned}
e^{\beta \Phi^{2}}= & e^{\beta_{\Lambda} \Phi^{2}} e^{K-\lambda\left(\Phi^{2}-A^{2}\right)^{2}}\left(1+f_{0}\right)\left[\left(e^{C\left(\Phi^{2}-B\right)}-e_{3}^{C\left(\Phi^{2}-B\right)}\right)\right. \\
& \left.\times\left(e^{D\left(\Phi^{2}-B\right)^{2}}-e_{2}^{D\left(\Phi^{2}-B\right)^{2}}\right)+f_{r}\right]
\end{aligned}
$$

where

$$
e_{i}^{x}=\int_{0}^{1} d t \frac{(1-t)^{i-1}}{(i-1) !} e^{t x} x^{i}
$$

We expand the above equation into five terms, the first of which becomes the leading term in $g_{\text {new }}$, the rest becoming $r_{\text {new }}$. We get the desired form of the leading term by making the choice $B=A^{\prime 2}$ and shifting $K, A, \lambda$ to eliminate the linear term:

$$
\begin{aligned}
K^{\prime} & =K-C^{2} / 4 \lambda+\log \left(1+f_{0}\right), \\
A^{\prime 2} & =A^{2}+C / 2 \lambda, \\
\lambda^{\prime} & =\lambda-D .
\end{aligned}
$$

In order to show the norm condition for the remainder, we need bounds on the $f_{i}$, which we obtain with the help of the identity $r^{(i)}\left(\Phi^{2}\right)=\bar{\varphi}^{-n} \partial_{\varphi}^{i} r\left(\Phi^{2}\right)$ used as follows:

$$
\begin{aligned}
\left|r^{(i)}\right|_{\Phi^{2}=A^{\prime 2}} & =\left|\bar{\varphi}^{-i}\left(\frac{\partial}{\partial \varphi}\right)^{i} r\right|_{\Phi^{2}=A^{\prime 2}} \leqq\left(A^{\prime}\right)^{-i}\left|\left(\frac{\partial}{\partial \varphi}\right)^{i} r\right|_{\sqrt{\lambda}, 0} w\left(A^{\prime}\right) \\
& \leqq O(1)\left(A^{\prime} h\right)^{-i}|r|_{\sqrt{\lambda}, h} \leqq O\left(\lambda^{1-\frac{i}{2}}\right) .
\end{aligned}
$$


This, along with (5.2), allows us to show

$$
\left.\begin{array}{l}
\left|f_{0}\right| \leqq O\left(\lambda^{1 / 2}\right) \\
\left|f_{1}\right| \leqq O(\lambda) \\
\left|f_{2}\right| \leqq O\left(\lambda^{3 / 2}\right)
\end{array}\right\} \Rightarrow \begin{aligned}
& C \leqq O(\lambda) \\
& D \leqq O\left(\lambda^{3 / 2}\right)
\end{aligned} .
$$

The derivative conditions for $r_{\text {new }}$ are obvious. To see the norm condition, we need the following

\section{Lemma 5.4.}

$$
\left.\mid \exp \left[-b_{1}\left(\Phi^{2}-B_{1}\right)^{2}+b_{2}\left(\Phi^{2}-B_{2}\right)\right]\left(\Phi^{2}-A^{\prime 2}\right)^{m}\right]_{0, \alpha g_{2}^{\prime}, A^{\prime}, h^{\prime}} \leqq c(m) \lambda^{-m / 2},
$$

where $b_{1}=\lambda+O\left(\lambda^{3 / 2}\right), b_{2}=O(\lambda),\left|B_{i}-A^{2}\right| \leqq O(1), g_{2}^{\prime}=4 \lambda^{\prime} A^{\prime 2}$, and $h^{\prime}=g_{2}^{\prime-1 / 2}$.

Proof. Let $F\left(\Phi^{2}\right)$ denote the function inside the norm in the statement. Using Corollary 4.5, we need to bound $\left|F(z) w(\varphi)^{-1}\right|$, with $z=|\varphi|^{2}+l, l \in C_{2 h}(\varphi), \varphi \in \mathbb{C}$. This is easiest to see if we pick some number $\Delta=O(1)$ (chosen large enough) and consider two regions separately:

(i) $\left.|| \varphi\right|^{2}-A^{\prime 2} \mid<\Delta \lambda^{-1 / 2}$. Here we have that $|l| \leqq O\left(\lambda^{-1 / 2}\right)$, so using the conditions in the statement on the $b_{i}, B_{i}$ as well as the definitions of $g_{2}^{\prime}$ and $w(\varphi)$ we see that both $|F(z)|$ and $w(\varphi)^{-1}$ are bounded by $O(1)$.

(ii) ||$\varphi\left|-A^{\prime 2}\right| \geqq \Delta \lambda^{-1 / 2}$. In this region, if $|\varphi|<h$, then

$$
\left|\frac{l}{|\varphi|^{2}-A^{\prime 2}}\right| \leqq \frac{O\left(h^{2}\right)}{\Delta \lambda^{-1 / 2}} \leqq O\left(\Delta^{-1}\right) .
$$

If $|\varphi| \geqq h$, then

$$
\left|\frac{l}{|\varphi|^{2}-A^{\prime 2}}\right| \leqq\left|\frac{O(h)|\varphi|}{|\varphi|^{2}-A^{\prime 2}}\right| .
$$

The function $|\varphi| /\left(|\varphi|^{2}-A^{\prime 2}\right)$, with $|\varphi|$ in the large-field region, achieves its maximum at $|\varphi|^{2}=A^{\prime 2}+\Delta \lambda^{-1 / 2}$. So

$$
\begin{aligned}
\left|\frac{l}{|\varphi|^{2}-A^{\prime 2}}\right| & \leqq \frac{O(h)\left(A^{\prime 2}+\Delta \lambda^{-1 / 2}\right)^{1 / 2}}{\Delta \lambda^{-1 / 2}} \\
& \leqq O\left(\Delta^{-1 / 2}\right)
\end{aligned}
$$

and we can write

$$
|F(z)| \leqq \exp \left[-b_{1} \kappa_{1}\left(|\varphi|^{2}-A^{\prime 2}\right)^{2}\right] \kappa_{2}^{m}\left(|\varphi|^{2}-A^{\prime 2}\right)^{m}
$$

where $\kappa_{i}=1+O\left(\Delta^{-1 / 2}\right)$. At this point we can use half the decay to cancel the weight function and half to kill the monomial, leaving us with the bound in the statement.

Lemma 5.4 applies directly to the terms in (5.5) containing an $e_{2}$ or $e_{3}$. To see that the term containing $f_{r}$ is also small enough in norm we apply Lemma 5.4 to the 
last two terms in (5.4) and then write

$$
f_{\geqq 3}=f-f_{0}-f_{1}\left(\Phi^{2}-A^{\prime 2}\right)-f_{2}\left(\Phi^{2}-A^{\prime 2}\right)^{2} .
$$

Lemma 5.4 applies to all of the terms but the one containing $f$; for this one, we need to norm the quantity

$$
e^{K-\lambda\left(\Phi^{2}-A^{\prime 2}\right)^{2}} \eta: \Phi^{6}:+e^{\left(\beta-\beta_{\Lambda}\right) \Phi^{2}} r .
$$

To handle the first term we expand the Wick monomial and then write $\Phi^{2}=\left(\Phi^{2}-A^{\prime 2}\right)+A^{\prime 2}$, applying Lemma 5.4 to each of the resultant terms. For the term containing the remainder we use

$$
\begin{aligned}
&\left|e^{\left(\beta-\beta_{\Lambda}\right) \Phi^{2}} r\right|_{K^{\prime}, \alpha g_{2}^{\prime}, A^{\prime}, h^{\prime}} \leqq O(1)\left|e^{\left(\beta-\beta_{\Lambda}\right) \Phi^{2}} r\right|_{0, \alpha g_{2}^{\prime}, A^{\prime}, h^{\prime}} \\
& \leqq O(1)\left|e^{\left(\beta-\beta_{\Lambda}\right) \Phi^{2}} r\right|_{\frac{1}{2} \sqrt{\lambda}, \lambda^{-1 / 4}} \\
& \leqq O(1)\left|e^{\left(\beta-\beta_{\Lambda}\right) \Phi^{2}}\right|_{-\frac{1}{2} \sqrt{\lambda}, \lambda^{-1 / 4}}|r|_{\sqrt{\lambda}, \lambda^{-1 / 4}} \\
& \leqq O(\lambda) .
\end{aligned}
$$

We have used Lemma 4.1, part (i), in the second line and the last line is true because we have assumed $\beta<\sqrt{\lambda} / 4$.

As long as $c_{2}=c_{2}(L)$ is chosen large enough, we have shown that the sum of the various terms we have claimed comprise $r_{\text {new }}$ satisfies the norm condition, and the proof of Proposition 5.3 is complete.

5.3. Intermediate Mass Flow. We now have a new representation $g_{\text {new }}$, and we drop the labels "new." It takes into consideration the fact that the minimum of the potential has moved far away from zero, which is reflected in the norm we use to measure the remainder; essentially we are expanding around $\Phi^{2}=A^{2}$ rather than $\Phi^{2}=0$. Eventually we will want to use this in our renormalization procedure, but in a narrow intermediate regime we must use essentially the old procedure of expanding $\mu_{C^{*}}$ as $1+\Delta_{C}+\cdots$ though there are a few differences.

We call the radial curvature of the potential at the minmum (the "radial mass") $g_{2}$, and note that $g_{2}=4 \lambda A^{2}$. So far we have been keeping track of the parameters $\beta, \lambda, \eta$ along with the function $r\left(\mu_{c}^{2}\right.$ is not a parameter since by definition it is just the mass along the critical trajectory). When we begin to do RGTs after the mass is well away from the critical point, we no longer need to keep track of $\eta$, since its role was to allow us to track the evolution of $\lambda$ carefully, which we only need to do very near the critical point. In the new representation, we keep track of the parameters $K, \lambda, A$ along with the function $r_{\text {new }}$. Theere is also a relation between these parameters and $r_{\text {new }}$ given by $g_{\text {new }}(0)=1$, due to $[\mathrm{BEI}]$ Theorem 4.2, which accounts for the fact that we are dispensing with $\eta$ and therefore losing a degree of freedom. It happens that it is more convenient to just track the evolution of $K, \lambda, A$ independently and not make use of the relation. We can also use $g_{2}=4 \lambda A^{2}$ to parameterize our recursion by $K, g_{2}, A$ instead, and we will use both parameterizations at various points in the sequel. 
Proposition 5.5. Let $g_{2} \in\left[L^{-2} \lambda^{1 / 2} / 5, \lambda^{1 / 4+v_{\delta}}\right]$, and suppose $e^{\beta_{\Lambda} \Phi^{2}} g$ satisfies I $A 2\left(\beta_{\Lambda}, K, \lambda, A, \alpha^{(n)}\right)$. Then $T\left(e^{\beta_{\Lambda} \Phi^{2}} g\right)$ satisfies $A 2\left(\beta_{\Lambda}^{\prime}, K^{\prime}, \lambda^{\prime}, A^{\prime}, \alpha^{(n+1)}\right)$, with

$$
\begin{aligned}
K^{\prime} & =L^{4} K+O\left(\lambda^{\frac{1}{4}+v_{\delta}}\right), \\
\lambda^{\prime} & =w_{\Lambda}^{4} \lambda+O\left(\lambda^{\frac{5}{4}+v_{\delta}}\right), \\
A^{\prime 2} & =\left(\frac{L}{w_{\Lambda}}\right)^{2} A^{2}+O(1), \\
\beta_{\Lambda}^{\prime} & =\frac{L^{2} \beta_{\Lambda}}{1-\beta_{\Lambda} \gamma} .
\end{aligned}
$$

Here $v_{\delta} \in(0,1 / 4), n$ is the number of times this Proposition has been applied since the switch of reresentations, and $\alpha^{(n)}$ was defined in (4.2).

Initially we do not know what $K^{\prime}, \lambda^{\prime}, A^{\prime}$ are. We will prove estimates under the assumption that

$$
\begin{aligned}
K^{\prime} & =L^{4} K+O\left(\lambda^{\varepsilon}\right), \\
\lambda^{\prime} & =w_{\lambda}^{4} \lambda+O\left(\lambda^{\varepsilon}\right), \\
A^{\prime 2} & =\left(\frac{L}{w_{A}}\right)^{2} A^{2}+O(1)
\end{aligned}
$$

for some $\varepsilon>0$. This will be justified in the course of the proof. We also set $\alpha \equiv \alpha^{(n)}$, $\alpha^{\prime} \equiv \alpha^{(n+1)}$.

As in the low-mass region, we define a quantity $\tilde{r}$ that will be used to absorb harmless remainder terms during the renormalization process. Here we take

$$
\begin{aligned}
|\tilde{r}|_{K^{\prime}, \alpha^{\prime}, g_{2}^{\prime}, A^{\prime}, h^{\prime}} \leqq L^{-2} c_{2} \sqrt{\lambda^{\prime}}, \\
\left|\tilde{r}^{(\alpha)}\right|_{\Phi^{2}=A^{\prime 2}} \leqq O(1) e^{K^{\prime}} \lambda^{2 v_{\delta}+\frac{1}{2}+\frac{\alpha}{2}},
\end{aligned}
$$

where in the second line we are considering $\tilde{r}$ to be a function of a single variable and differentiating $\alpha$ times with respect to it. Note that we do not yet know that $A^{\prime}$ is, only the assumption (5.7).

Again as in the low-mass region, we write

$$
\begin{aligned}
T\left(e^{\beta_{\Lambda} \Phi^{2}} g\right) & =e^{\frac{L^{2} \beta_{\Lambda}}{1-\beta_{\Lambda} \gamma} \Phi^{2}}\left(\mathscr{R} \mu_{\Gamma W_{\Lambda}} * g^{\mathscr{G}_{1}}\right)\left(W_{\Lambda} \Phi\right), \\
W_{\Lambda} & \equiv\left(1-\beta_{\Lambda} \Gamma\right)^{-1}, \\
g^{\mathscr{G}_{1}} & =S_{0}+S_{1}+S_{\geqq 2},
\end{aligned}
$$

where $S_{0}$ is the term with no remainders, etc. We can use Lemma 4.3 to handle most of this in the same manner as in the low-mass region: e.g. for the $S_{\geqq 2}$ term,

$$
\begin{aligned}
& \left|\mathscr{R} \mu_{\Gamma W_{A}} * S_{\geqq 2}\left(W_{\Lambda} \cdot\right)\right|_{K^{\prime}, \alpha^{\prime} g_{2}^{\prime}, A^{\prime}, h^{\prime}}=\left|\mu_{\Gamma} * S_{\geqq 2}(\cdot)\right|_{\frac{K^{\prime}}{L^{4}}}, \frac{\alpha^{\prime} g_{2}^{\prime}}{L^{2} w_{A}^{2}}, \frac{w_{A A^{\prime}}}{L}, \frac{w_{A} h^{\prime}}{L} \\
& \leqq O(1)\left|\mu_{\Gamma} * S_{\geqq 2}\right|_{K}, \frac{\alpha^{\prime} g_{2}^{\prime}}{L^{2} w_{A}^{2}},(1+c \lambda), A, \frac{w_{A} h^{\prime}}{L} \\
& \leqq O(1)\left|S_{\geqq 2}\right|_{K, \alpha g_{2}, A, \frac{w_{A} h^{\prime}}{L}} \\
& \leqq O(1)\left|S_{\geqq 2}\right|_{K, \alpha g_{2}, A, h} \\
& \leqq O(1) \sum_{|X| \geqq 2}|r|_{K, \alpha g_{2}, A, h}^{|X|}\left|g_{0}\right|_{K, \alpha g_{2}, A, h}^{\mathscr{G}_{1} \backslash X} \\
& \leqq c(L) O(\lambda)
\end{aligned}
$$


where $g_{0}$ is $g$ with $r$ set to 0 , and $c=O(1)$. We have used Lemma 4.3 in all but the last line, and Lemma 5.4 in the last line. Since $c(L)$ can be bounded by $\lambda^{\varepsilon}$, this yields the first of the $\tilde{r}$ estimates. The second follows by applying Corollary 4.7 to $S \geqq 2$.

We next claim that $\mathscr{R} \mu_{\Gamma W_{A}} * S_{1}$ is $\tilde{r}$. We first write

$$
\mathscr{R} \mu_{\Gamma W_{\Lambda}} * S_{1}=\mathscr{R} S_{1}+\mathscr{R} \int_{0}^{1} d t \mu_{t \Gamma W_{\Lambda}} * \Delta_{\Gamma W_{A}} S_{1} .
$$

By arguments similar to those above, we can show the second term is $\tilde{r}$. The first term will require a new argument. We write it as

$$
\begin{aligned}
\mathscr{R} S_{1}\left(W_{A} \Phi\right) & =F\left(\left(\frac{w_{A}}{L}\right)^{2} \Phi^{2}\right), \\
F(z) & =L^{4} e^{-\left(L^{4}-1\right) v(z)} r(z), \\
v(z) & =-K+\lambda\left(z-A^{2}\right)^{2} .
\end{aligned}
$$

We start by showing the second of the $\tilde{r}$ conditions. It is helpful to write

$$
r(z)=\frac{1}{2} \int_{0}^{1} d t(1-t)^{2} r^{\prime \prime \prime}\left(t\left(z-A^{2}\right)+A^{2}\right)\left(z-A^{2}\right)^{3},
$$

where we have used the inductive assumption on the vanishing of the derivatives of $r$. In bounding this and its first two derivatives we use our assumptions (5.7) along with Corollary 4.7, which tells us that

$$
\left|r^{(\alpha)}\right|_{A^{\prime 2} / L^{2}} \leqq O\left(\lambda^{1 / 2+\alpha / 2}\right) e^{K} .
$$

The derivatives can also act on the exponential, which brings down a power of $\lambda$ for $\alpha=1$ or 2 . Hence we have that

$$
\left|\left(\frac{\partial}{\partial \Phi^{2}}\right)^{\alpha} \mathscr{R} S_{1}\right|_{\Phi^{2}=A^{\prime 2}} \leqq O\left(\lambda^{2}\right) e^{K^{\prime}},
$$

which gives us the second $\tilde{r}$ estimate.

We still need to show the first $\tilde{r}$ estimate, the norm condition, for which we prove the following.

Lemma 5.6. Suppose $X(\Phi)=Y\left(\Phi^{2}\right)$ satisfies $|X|_{K, \alpha g_{2}, A, h}<\infty$, and $Y$ is a function of a single variable satisfying $\left.Y^{(\alpha)}\right|_{A^{2}}=0$ for $\alpha<m$, some $m \geqq 0$. Let

$$
Z(\Phi) \equiv X\left(\Phi_{x}\right)\left(e^{-v(\Phi)}\right)^{\mathscr{S}_{1} \backslash\{x\}}
$$

for $x \in \mathscr{G}_{1}$. Then

$$
\left|\mathscr{R} Z\left(W_{A} \cdot\right)\right|_{K^{\prime}, \alpha^{\prime} g_{2}^{\prime}, A^{\prime}, h^{\prime}} \leqq O\left(L^{-2 m}\right)|X|_{K, \alpha g_{2}, A, h} .
$$

Proof. We first observe that $\mathscr{R} Z$ is analytic in a sufficiently large region to apply Corollary 4.5 , since

$$
\begin{aligned}
\left|\mathscr{R} Z\left(W_{A} \cdot\right)\right|_{K^{\prime}, \alpha^{\prime} g_{2}^{\prime}, A^{\prime}, h^{\prime}} & \leqq O(1)|Z|_{K, \alpha g_{2}, A, \frac{c w_{A}}{L} h^{\prime}} \\
& \leqq O(1)|Z|_{K, \alpha g_{2}, A, h} \\
& \leqq c(L)|X|_{K, \alpha g_{2}, A, h}
\end{aligned}
$$


where the second line is true for any $c<L / w_{\Lambda}$, since $h^{\prime} \leqq h$. So we can certainly choose $c$ large so $\mathscr{R} Z\left(W_{A} z\right)$ is analytic for $z \in R_{2 h^{\prime}}$. To apply Corollary 4.5 we must bound $F(z) w^{\prime}(\varphi)^{-1}$, where

$$
\begin{aligned}
F(z) & =e^{-\left(L^{4}-1\right) v(z)} Y(z) \\
z & =\left(\frac{w_{A}}{L}\right)^{2}\left(|\varphi|^{2}+l\right) \quad l \in C_{2 h^{\prime}}(\varphi) .
\end{aligned}
$$

We handle the bound in slightly different ways depending on the value of $|\varphi|^{2}$. Recall that $\left|l_{i}\right| \leqq \max \left(32 h^{\prime 2}, 16 h^{\prime}|\varphi|\right)$. We call the region of

$$
\left.\left|\left(\frac{w_{A}}{L}\right)^{2}\right| \varphi\right|^{2}-A^{2} \mid \geqq\left(\frac{w_{A}}{L}\right)^{2} \Delta \lambda^{-1 / 2}
$$

with $\Delta>1$, chosen large enough, to be the large-field region. In this region, if $|\varphi| \geqq 16 h^{\prime}$, then

$$
\begin{aligned}
l\left(\frac{w_{A}}{L}\right)^{2}\left(\left(\frac{w_{A}}{L}\right)^{2}|\varphi|^{2}-A^{2}\right)^{-1} & \leqq 16 h^{\prime} \frac{|\varphi|}{|\varphi|^{2}-\left(\frac{L}{w_{A}}\right)^{2} A^{2}} \\
& \leqq \frac{16 h^{\prime}}{\Delta \lambda^{-1 / 2}}\left(\left(\frac{L}{w_{A}}\right)^{2} A^{2}+\Delta \lambda^{-1 / 2}\right)^{1 / 2} \\
& \leqq O\left(\Delta^{-1 / 2}\right) .
\end{aligned}
$$

We obtained the last line by noting that if $\Delta \lambda^{-1 / 2}>L^{2} A^{2} / w_{\lambda}^{2}$, then we can use $h^{\prime} \leqq h \leqq \lambda^{-1 / 4}$ to get the bound of $O\left(\Delta^{-1 / 2}\right)$, where as if $\Delta \lambda^{-1 / 2}<L^{2} A^{2} / w_{A}^{2}$, then we are in the regime where $h^{\prime}=O(h / L)$ and we can use the fact that $h A=\lambda^{-1 / 2} / 2$ to get the bound $O\left(\Delta^{-1}\right) \leqq O\left(\Delta^{-1 / 2}\right)$. Finally, if $|\varphi|<16 h^{\prime}$, we see directly that the estimate $O\left(\Delta^{-1}\right)$ applies, so (5.10) applies for the entire large-field region. This allows us to write

$\left(\frac{w_{A}}{L}\right)^{2}|\varphi|^{2}-A^{2}+\left(\frac{w_{A}}{L}\right)^{2} l=\left(\left(\frac{w_{A}}{L}\right)^{2}|\varphi|^{2}-A^{2}\right)\left(1+O\left(\Delta^{-1 / 2}\right)\right)$.

We also have

$$
Y(z)=\int_{0}^{1} \frac{(1-t)^{m-1}}{(m-1) !} Y^{(m)}\left(t\left(z-A^{2}\right)+A^{2}\right)\left(z-A^{2}\right)^{m} .
$$

Using Corollary 4.7 to bound the derivatives of $Y$, and inserting (5.11), we obtain

$$
\begin{aligned}
\mid F(z) w^{\prime}(\varphi)^{-1} & \leqq \exp \left[\left(L^{4}-1\right)\left(K-\kappa \lambda\left(\left(\frac{w_{A}}{L}\right)^{2}|\varphi|^{2}-A^{2}\right)^{2}\right)\right] \\
& \times e^{K} O\left(\lambda^{m / 2}\right)|X|_{K, \alpha g_{2}, A, h}\left|z-A^{2}\right|^{m} w^{\prime}(\varphi)^{-1} \\
& \leqq O\left(L^{-2 m}\right)|X|_{K, \alpha g_{2}, A, h} .
\end{aligned}
$$

with $\kappa=1+O\left(\Delta^{-1 / 2}\right)$. We used half of the exponential to kill the weight function and half to kill the polynomial in $z$. 
In the small-field region, $l$ is comparable in magnitude to $w_{A}^{2}|\varphi|^{2} / L^{2}-A^{2}$, so we can no longer get any decay from the $e^{-v}$, but nor do we need it. From

$$
\left(\frac{w_{A}}{L}\right)^{2}|l| \leqq O\left(L^{-2}\right) \lambda^{-1 / 2}
$$

we see

$$
\left.\left|\left(\frac{w_{A}}{L}\right)^{2}\right| \varphi\right|^{2}-A^{2}+\left(\frac{w_{A}}{L}\right)^{2} l \mid \leqq O\left(L^{-2}\right) \lambda^{-1 / 2} .
$$

Since $w^{\prime}(\varphi)^{-1} \leqq O(1)$ in this region, we have the same bound as before.

We are left with the $S_{0}$ term. We relegate most of it to $\tilde{r}$ by writing

$$
\mathscr{R} \mu_{\Gamma W_{A}} * g_{0}^{\mathscr{S}_{1}}=\left[1+\Delta+\int_{0}^{1} d t(1-t) \mu_{t \Gamma W_{A}} * \Delta^{2}\right] g_{0}^{\mathscr{S}_{1}}
$$

and claiming the last term is $\tilde{r}$. The proof is an application of Lemma 4.3; i.e. to get the norm condition we write

$$
\begin{aligned}
\left.\mathscr{R} \mu_{\Gamma W_{A}} *\left(\Delta^{2} S_{0}\right)(W \cdot)\right|_{K^{\prime}, \alpha^{\prime}, g^{\prime}, A^{\prime}, h^{\prime}} & \leqq c(L) h^{-4} \\
& \leqq L^{-2} c_{2} \sqrt{\lambda^{\prime}},
\end{aligned}
$$

where the last line is true since we have chosen $v_{\delta}>0$., To get the derivative condition we use $F^{(n)}\left(\Phi^{2}\right)=\bar{\varphi}^{-n} \partial_{\varphi}^{n} F\left(\Phi^{2}\right)$, and again Lemmas 4.1 and 4.3 show

and so

$$
e^{-K^{\prime}}\left|\partial_{\varphi}^{n} \mathscr{R} \mu_{t \Gamma W_{A}} * \Delta^{2} S_{0}\right|_{w^{2} \varphi \bar{\varphi}=A^{\prime 2}} \leqq c(L) h^{-4-n}
$$

$$
e^{-K^{\prime}}\left|\partial_{\Phi^{2}}^{n} \mathscr{R} \mu_{t \Gamma W_{A}} * \Delta^{2} S_{0}\right|_{w^{2} \varphi \bar{\varphi}=A^{\prime 2}} \leqq \lambda^{(1+n) / 2+2 v_{\delta}} .
$$

This is the second of the $\tilde{r}$ conditions. So we are left with

$$
\begin{aligned}
\mathscr{R} \mu_{\Gamma W_{A}} * g^{\mathscr{S}_{1}}= & (1+\Delta) S_{0}+\tilde{r} \\
= & \exp \left[L^{4} K-\lambda w_{A}^{4}\left(\Phi_{0}^{2}-\left(\frac{L}{w_{A}}\right)^{2} A^{2}\right)^{2}\right] P\left(w_{A} \Phi_{0}^{2}\right), \\
P\left(\Phi^{2}\right)= & 1+\gamma w_{A} L^{4}\left(4 \lambda^{2}\left(\left(\frac{w_{A}}{L}\right)^{2} \Phi_{0}^{2}-A^{2}\right)^{3}\right. \\
& +4 \lambda^{2} A^{2}\left(\left(\frac{w_{A}}{L}\right)^{2} \Phi_{0}^{2}-A^{2}\right)^{2} \\
& \left.-2 \lambda\left(\left(\frac{w_{A}}{L}\right)^{2} \Phi_{0}^{2}-A^{2}\right)-2 \lambda A^{2}\right) .
\end{aligned}
$$

The cubic term is $\tilde{r}$, as long as we take $c_{2}$ large enough, as is easily seen from Lemma 5.4. We denote by $P_{<3}$ the terms in $P$ that are at most quadratic. We write

$$
\begin{aligned}
\left(\mathscr{R} \mu_{\Gamma} W_{\Lambda} g^{\left(G_{1}\right.}\right)\left(W_{A} \Phi\right) & =e^{L^{4} K-\lambda w_{A}^{\prime}\left(\Phi^{2}-\frac{L^{2} A^{2}}{w_{A}^{2}}\right)^{2}}\left(1+f^{(1)}+f^{(2)}\right), \\
f^{(1)} & \equiv P_{<3}-1, \\
f^{(2)} & \equiv e^{-L^{4} K+\lambda w_{A}^{4}\left(\Phi^{2}-\frac{L^{2} A^{2}}{w_{A}^{2}}\right)^{2}} \tilde{r},
\end{aligned}
$$


where $\tilde{r}$ is the sum of $\tilde{r}$ terms we have accumulated up until now. We now can proceed in a manner similar to what we did following (5.3) by setting $f=f^{(1)}+f^{(2)}$. It is important to notice that $f \geqq 3=0$, so the $f \geqq 3$ contribution to $f_{r}$ comes only from $f^{(2)}$. This fact, along with the assumption (5.8), allows us to see that $f_{r}$ is small enough in norm. We can then read off

$$
\begin{aligned}
K^{\prime} & =L^{4} K-\frac{C^{2}}{4 \lambda}+\log \left(1+f_{0}\right), \\
\lambda^{\prime} & =w_{A}^{4} \lambda-4 \lambda^{2} A^{2} \gamma w_{A}^{5}+O\left(\lambda^{\frac{3}{2}+2 v_{\delta}}\right), \\
A^{\prime 2} & =\left(\frac{L}{w_{A}}\right)^{2} A^{2}+\frac{C}{2 \lambda} .
\end{aligned}
$$

Also, noting that $g_{2}^{\prime}=4 \lambda^{\prime} A^{\prime 2}$, we have

$$
g_{2}^{\prime}=\frac{L^{2} w_{A}^{2} g_{2}}{1+g_{2} \gamma w_{A}}\left(1+O\left(\lambda^{\frac{1}{2}+2 v_{s}}, A^{-2}\right)\right) .
$$

The proof of Proposition 5.5 is complete.

5.4. Large Mass Flow. At this point it becomes more useful to parameterize our recursion in terms of $g_{2}$ rather than $\lambda$, since here the main behavior comes from the first term in

$$
\lambda\left(|\varphi|^{2}-A^{2}\right)^{2}=g_{2}(|\varphi|-A)^{2}+\frac{g_{2}}{A}(|\varphi|-A)^{3}+\frac{g_{2}}{4 A^{2}}(|\varphi|-A)^{4}
$$

while the others are small corrections because $A \gg 1$. We retain the same definition of the representation $g$ because $\exp \left(-g_{2}(|\varphi|-A)^{2}\right)$ by itself does not have very nice analyticity properties, a fact that would have to be compensated for by the remainder, which would be undesirable as we would like the remainder by itself to have nice analyticity properties

Proposition 5.7. Let $g_{2}>\lambda^{1 / 4+v_{\delta}}$, and suppose $\exp \left(\beta_{\Lambda} \Phi^{2}\right) g$ satisfies IA2 $\left(\beta_{\Lambda}, K, \lambda, A, \alpha^{(n)}\right)$. Then $T\left(e^{\beta_{\Lambda} \Phi^{2}} g\right)$ satisfies IA2 $\left(\beta_{\Lambda}^{\prime}, K^{\prime}, \lambda^{\prime}, \alpha^{(n+1)}\right)$, with

$$
\begin{aligned}
K^{\prime} & =L^{4} K-\frac{1}{2} L^{4} \log \left(1+g_{2} \gamma w_{A}\right)+O\left(L^{4}\right), \\
A^{\prime 2} & =\left(\frac{w_{A}}{L}\right)^{2} A^{2}+O(1), \\
\lambda^{\prime} & =\frac{w_{A}^{4} \lambda}{1+g_{2} \gamma w_{A}}\left(1+O\left(\frac{g_{2}}{A}\right)\right), \\
\beta_{\Lambda}^{\prime} & =\frac{L^{2} \beta_{A}}{1-\beta_{\Lambda} \gamma} .
\end{aligned}
$$

Here $n$ is the number of times this Proposition or Proposition 5.5 have been applied since the switch of representations, and $\alpha^{(n)}$ was defined in (4.2).

Initially we do not know what $K^{\prime}, \lambda^{\prime}$, and $A^{\prime}$ are. We will prove estimates under the assumption that they take on the values (5.12), which will be justified in the 
course of the proof. We also set $\alpha \equiv \alpha^{(n)}, \alpha^{\prime} \equiv \alpha^{(n+1)}$, and notice that $g_{2}$ approaches a fixed point value of order $L^{2}$, since

$$
g_{2}^{\prime}=4 \lambda^{\prime} A^{\prime 2}=\frac{L^{2} w_{\Lambda}^{2} g_{2}}{1+g_{2} \gamma w_{A}}\left(1+O\left(\frac{g_{2}}{A}\right)\right) \text {. }
$$

As before, we accumulate error terms in $\tilde{r}$, now defined by

$$
\begin{gathered}
|\tilde{r}|_{K^{\prime}, x^{\prime}, g_{2}^{\prime}, A^{\prime}, h^{\prime}} \leqq L^{-2} c_{2} \sqrt{\lambda^{\prime}}, \\
\left|\tilde{r}^{(\alpha)}\right|_{\Phi^{2}=A^{\prime 2}} \leqq O(1) e^{K^{\prime}} \lambda^{1 \frac{\alpha}{2}} .
\end{gathered}
$$

We begin by writing $g^{\mathscr{G}_{1}}=S_{0}+S_{1}+S_{\geqq 2}$, and $S_{2}$ goes mainly as before:

$$
\begin{aligned}
\left|\mathscr{R} \mu_{\Gamma W_{A}} * S_{\geqq 2}\left(W_{A} \cdot\right)\right|_{K^{\prime}, \alpha^{\prime} g_{2}^{\prime}, A^{\prime}, h^{\prime}} & \leqq e^{\int d x d y\left|\Gamma W_{A}(x, y)\right| h^{-2}}\left|S_{\geqq 2}\right|_{K, \alpha g_{2}, A, h} \\
& \leqq e^{O\left(\gamma w_{A} L^{6}\right)}\left|S_{\geqq 2}\right|_{K, \alpha g_{2}, A, h} \\
& \leqq e^{O\left(\gamma w_{A} L^{6}\right)} O(\lambda) \\
& \leqq L^{-2} c_{2} \sqrt{\lambda^{\prime}}
\end{aligned}
$$

where we have used that $h$ stays bounded from below by $O\left(L^{-1}\right)$, since $g_{2}$ approaches a fixed point value. This gives us the first $\tilde{r}$ estimate, and the second follows exactly as before. So we have that

$$
T\left(e^{\beta_{\Lambda} \Phi^{2}} g\right)=e^{\beta_{\Lambda}^{\prime} \Phi^{2}}\left[\mathscr{R} \mu_{\Gamma W_{\Lambda}} *\left(S_{0}+S_{1}\right)+\tilde{r}\right] .
$$

To handle the other two terms, our basic strategy will be to write

$$
\mathscr{R} \mu_{\Gamma W_{A}} *\left(S_{0}+S_{1}\right)=e^{\tilde{K}-\tilde{\lambda}\left(\Phi^{2}-\tilde{A}^{2}\right)^{2}}+\tilde{R}
$$

and to show that $\tilde{R}$ satisfies $\tilde{r}$ estimates for some choice of $\tilde{K}, \tilde{\lambda}, \tilde{A}$.

Set $F(\Phi)=\left(\mathscr{R} \mu_{\Gamma W_{A}} * g^{\mathscr{G}} 1\right)\left(W_{A} \Phi\right)$. We apply Lemma 4.3 to find $|F|_{K^{\prime}, \alpha^{\prime}, g^{\prime}, A^{\prime}, c h^{\prime}}<$ $\infty$ if $c \leqq L / 2 w_{A}$, say. Now, by Theorem 4.2 of [BEI], we know that $F$ is a function of $\Phi^{2}$ alone, so we can project down to the degree-zero component of $\mathbb{G}$ and consider the function $f(\varphi, \bar{\varphi}) \equiv F(\varphi \bar{\varphi})$. Clearly the purely bosonic norm $|f|_{K^{\prime}, \alpha^{\prime} g_{2}^{\prime}, A^{\prime}, c h^{\prime}}$ is bounded above by the combined fermionic-bosonic norm $|F|_{K^{\prime}, \alpha^{\prime} g_{2}^{\prime}, A^{\prime}, c h^{\prime}}$, and hence $f$ is analytic in $\varphi, \bar{\varphi}$ with a radius of convergence of at least $c h^{\prime}$ everywhere, which implies that $f(z)$ is analytic in the interior of $\bigcup_{\varphi \in \mathbb{C}} D_{c h^{\prime}}^{(1)}(\varphi) \supset R_{2 h^{\prime}}$, if $c$ (and $L$ ) is large enough. The same argument applies to $\mathscr{R} \mu_{\Gamma W_{A}} * S \geqq 2$, so we conclude that $\widetilde{R}(z)$ is analytic in $R_{2 h^{\prime}}$, and hence we can apply Corollry 4.5 to it; we need only bound $\tilde{R}\left(|\varphi|^{2}+l\right)$ for $\varphi \in \mathbb{C}, l \in C_{2 h^{\prime}}(\varphi)$. In particular, note that we have projected onto the degree-zero component of $\mathbb{G}$; i.e. when we evaluate $\widetilde{R}\left(\Phi^{2}\right)$ we can set $\psi=\bar{\psi}=0$, which simplifies our calculations. Also, at this point we further simplify by specializing to our specific form of $\Gamma(x, y)$, namely $\gamma \delta(x, y)$.

We begin by defining $\varphi^{\prime} \equiv \varphi+y, \bar{\varphi}^{\prime} \equiv \bar{\varphi}+\bar{y}$, where $y, \bar{y} \in \mathbb{C}$ are such that $\varphi^{\prime} \bar{\varphi}^{\prime}=\varphi \bar{\varphi}+l$ and $|y|,|\bar{y}| / 2 w_{\Lambda}$. We use this to write

$$
\begin{aligned}
& P\left(|\varphi|^{2}+l\right) \equiv \int d \mu_{\gamma w_{A}}(\zeta) e^{K-\lambda\left(\left|\frac{w_{A} \varphi^{\prime}}{L}+\zeta^{B}\right|^{2}+\zeta^{F} \bar{\zeta}^{F}\right)^{2}}, \\
& Q\left(|\varphi|^{2}+l\right) \equiv \int d \mu_{\gamma w_{A}}(\zeta) r\left(\left|\frac{w_{\Lambda} \varphi^{\prime}}{L}+\zeta^{B}\right|^{2}+\zeta^{F} \bar{\zeta}^{F}\right),
\end{aligned}
$$


where $\zeta$ is the fluctuation superfield, with bosonic and fermionic components $\left(\zeta^{B}, \zeta^{F}\right)$, and by $|u|^{2}$ we mean $u \bar{u}$ even when $u, \bar{u}$ are not complex conjugates, as is the case here with $\varphi^{\prime}, \bar{\varphi}^{\prime}$.

Hence,

$$
\mathscr{R} \mu_{\Gamma W_{A}} *\left(S_{0}+S_{1}\right)\left(w_{\Lambda}^{2}\left(|\varphi|^{2}+l\right)\right)=P^{L^{d}}+L^{d} P^{L^{d}-1} Q+\tilde{r} .
$$

We further subdivide by expanding in power series in $\zeta^{F} \bar{\zeta}^{F}$, which have only two terms in this case:

$$
\begin{aligned}
& P_{0}(\varphi \bar{\varphi}+l) \equiv \int d \mu_{\gamma w_{A}}\left(\zeta^{B}\right) e^{K-\lambda\left(\left|\frac{w_{A} \varphi^{\prime}}{L}+\zeta^{B}\right|^{2}-A^{2}\right)^{2}}, \\
& P_{F}(\varphi \bar{\varphi}+l) \equiv \int d \mu_{\gamma w_{A}}\left(\zeta^{B}\right) d \mu_{\gamma w_{A}}\left(\zeta^{F}\right) e^{K-\lambda\left(\left|\frac{w_{A} \varphi^{\prime}}{L}+\zeta^{B}\right|^{2}-A^{2}\right)^{2}} \\
& \times(-2 \lambda)\left(\left|\frac{w_{A} \varphi^{\prime}}{L}+\zeta^{B}\right|^{2}-A^{2}\right) \zeta^{F} \bar{\zeta}^{F} \\
& Q_{0}(\varphi \bar{\varphi}+l) \equiv \int d \mu_{\gamma w_{A}}\left(\zeta^{B}\right) r\left(\left|\frac{w_{A} \varphi^{\prime}}{L}+\zeta^{B}\right|^{2}\right), \\
& Q_{F}(\varphi \bar{\varphi}+l) \equiv \int d \mu_{\gamma w_{A}}\left(\zeta^{B}\right) d \mu_{\gamma w_{A}}\left(\zeta^{F}\right) r^{\prime}\left(\left|\frac{w_{A} \varphi^{\prime}}{L}+\zeta^{B}\right|^{2}\right) \zeta^{F} \bar{\zeta}^{F} .
\end{aligned}
$$

We will choose $\tilde{K}, \tilde{\lambda}, \tilde{A}$ to be of the form (5.12); the precise values of the $O(\cdot)$ corrections will be determined below. We will deal with two regions of $\varphi$ space in different ways; the large-field region, when $\varphi$ is far away from its minmum, has very little weight, which we can use to bound its contribution, while the small-field region is amenable to perturbative expansions.

Large-field analysis. We define the large-field region to be

$$
\left|\frac{w_{\Lambda}|\varphi|}{L}-A\right|>h A^{\varepsilon}
$$

Lemma 5.8. If $|u|,|\bar{u}| \in \mathbb{C}$ not necessarily complex conjugates, then for all $z \in \mathbb{C}$,

$$
\left|e^{-\lambda\left(|z+u|^{2}-A^{2}\right)^{2}}\right| \leqq O(1) e^{-b g_{2}(|z|-A)^{2}}
$$

for some $(1 / 4)-\varepsilon<b<1 / 4$.

Proof. Pick some $\Delta>0$ large enough. Then, if ||$z|-A| \leqq \Delta h$, the left-hand side of (5.15) is bounded above by $O(1)$, while the right-hand side is bounded below by $O(1)$. If ||$z|-A|>\Delta h$, we bound the left-hand side of (5.15) by

$$
e^{-\left(1-O\left(\Delta^{-1}\right)\right) \lambda\left(|z|^{2}-A^{2}\right)} \leqq e^{-b g_{2}(|z|-A)^{2}}
$$

for $b=\left(1-O\left(\Delta^{-1}\right)\right) / 4$.

We apply this to $P_{0}$ by setting

$$
z=\frac{w_{A} \varphi}{L}+\zeta^{B}, \quad \bar{z}=\frac{w_{A} \bar{\varphi}}{L}+\bar{\zeta}^{B}
$$


and, choosing $b$ near to $1 / 4$, find

$$
\begin{aligned}
\left|P_{0}\right| \leqq & \int d \mu_{\gamma w_{A}}\left(\zeta^{B}\right) \mid e^{K-\lambda\left(\left|z+\frac{w_{A} \varphi^{\prime}}{L}+\zeta^{B}\right|^{2}-A^{2}\right)^{2} \mid} \\
\leqq & O(1) \int d \mu_{\gamma w_{A}}\left(\zeta^{B}\right) e^{K-b g_{2}(|z|-A)^{2}} \\
\leqq & O(1) e^{K}\left(\frac{\left(\gamma w_{A}\right)^{-1}|\varphi| / L+b g_{2} A}{\left[\left(\gamma w_{A}\right)^{-1}+b g_{2}\right]^{3 / 2}}\right) \\
& \times \exp \left(\frac{-b g_{2}}{1+b g_{2} \gamma w_{A}}\left(\frac{w_{A}|\varphi|}{L}-A\right)^{2}\right)
\end{aligned}
$$

where to obtain the last line, we have shifted $\zeta^{B} \rightarrow \zeta^{B}-w_{\Lambda} \varphi / L$. Now we use the fact that $b-\alpha \geqq O(1)$. Choose $\tilde{b} \equiv \alpha+(b-\alpha) / 2$, and use part of the exponential to kill the powers in front, valid for $\varphi$ in the large-field region. We conclude

$$
\left|P_{0}(\varphi \bar{\varphi}+l)\right| \leqq O(1) e^{K-\frac{\tilde{b} g_{2}}{1+b g_{2} \gamma w_{A}}\left(\frac{w_{A}|\varphi|}{L}-A\right)^{2}-O\left(A^{2 \varepsilon}\right)} .
$$

The $P_{F}$ term goes almost as the $P_{0}$ term; the only differences are a factor of $\gamma w_{A}$ from the fermionic fluctuation integral and the extra factor of $-2 \lambda\left(\left|z+w_{A} y / L\right|^{2}-A^{2}\right)$ inside the integral. The latter can be dominated by the exponential at a cost of a fraction of the decay, which we can easily afford, and a factor of $\lambda^{-1 / 2}$. We are left with

$$
\left|P_{F}(\varphi \bar{\varphi}+l)\right| \leqq O\left(\lambda^{1 / 2}\right) e^{K-\frac{\tilde{b} g_{2}}{1+b g_{2} \gamma w_{A}}\left(\frac{w_{A}|\varphi|}{L}-A\right)^{2}-O\left(A^{2 c}\right)} .
$$

for $\varphi$ in the large-field region.

We only need very crude bounds on the remaining terms $Q_{0}$ and $Q_{F}$, as we can get all of the decay we need from the $P$ 's. So we just apply Corollary 4.7 to $r\left(\left|z+w_{A} y / L\right|^{2}\right)$, retaining the definitions (5.16), and find

$$
\begin{aligned}
& \left|Q_{0}(\varphi \bar{\varphi}+l)\right| \leqq O\left(\lambda^{1 / 2}\right) e^{K}, \\
& \left|Q_{F}(\varphi \bar{\varphi}+l)\right| \leqq O(\lambda) e^{K},
\end{aligned}
$$

which is true for all values of $\varphi$.

The next observation we need to make in the large-field region is that, from Lemma 5.8, we have

$$
\begin{aligned}
\left|e^{\tilde{K}-\tilde{\lambda}\left(\left|\varphi^{\prime}\right|^{2}-\tilde{A}^{2}\right)^{2}}\right| & \leqq O(1) e^{\tilde{K}-b \tilde{g}_{2}(|\varphi|-\tilde{A})^{2}} \\
& \leqq e^{\tilde{K}-\tilde{b} \tilde{g}_{2}(|\varphi|-\tilde{A})^{2}-O\left(A^{2 \varepsilon}\right)}
\end{aligned}
$$

for $\varphi$ in the large-field region. Again, we have lots of decay to spare, of which we have used part to produce a factor exponentially small in $A$ and part to produce a term that will kill the weight function.

The final step to complete the bound in the large field region is to combine the estimates (5.18)-(5.19): due to our choice of $b$ we see that we have plenty of room in the decay to shift $L A / w_{A} \rightarrow A^{\prime}$ and that the $L^{4}-1$ factors of $P$ have enough decay to compensate for the fact that we have not kept track of any from the $Q$ terms. We find

$$
|\tilde{R}(\varphi \bar{\varphi}+l)| \leqq e^{-O\left(A^{2 \tau}\right)+K^{\prime}-\alpha^{\prime} g_{2}^{\prime}\left(|\varphi|-A^{\prime}\right)^{2}}
$$


for $\varphi$ in the large-field region, which, along with analogous bounds in the smallfield region, will be enough to obtain the $\tilde{r}$ norm condition from Corollary 4.5.

The second of the $\tilde{r}$ estimates, the derivative conditions, involves evaluating $\tilde{R}$ at a point which is well outside the large field region, so we do not need to deal with it yet.

Small-Field Analysis. Next we attack the small-field region, when $\left|w_{\Lambda}\right| \varphi|/ L-A| \leqq$ $h A^{\varepsilon}$. Here it will be convenient to define

$$
\begin{aligned}
\sigma & \equiv \frac{w_{A}}{L}\left(|\varphi|^{2}+l\right)^{1 / 2}-A, \\
\frac{w_{A} \varphi_{0}}{L} & \equiv(\sigma+A) \hat{\varphi}, \\
\frac{w_{\Lambda} \bar{\varphi}_{0}}{L} & \equiv(\sigma+A) \hat{\bar{\varphi}},
\end{aligned}
$$

so $\varphi_{0} \bar{\varphi}_{0}=\varphi \bar{\varphi}+l$. We see that $|\sigma| \leqq O\left(h A^{\varepsilon}\right)$ when $\varphi$ is in the small-field region. We also write the bosonic fluctuation field in a new basis:

$$
\zeta^{B}=\tilde{\sigma} \hat{\varphi}+i \tilde{\pi} \hat{\varphi} ; \quad \bar{\zeta}^{B}=\tilde{\sigma} \hat{\bar{\varphi}}-i \tilde{\pi} \hat{\bar{\varphi}} .
$$

Putting these definitions together, we have

$$
\left|\frac{w_{\Lambda} \varphi_{0}}{L}+\zeta^{B}\right|^{2}=(A+\sigma+\tilde{\sigma})^{2}+\tilde{\pi}^{2} .
$$

We use this in considering the integral

$$
I \equiv \int d \mu_{\gamma w_{\Lambda}}\left(\zeta^{B}\right) e^{-\lambda\left(\left|\frac{w_{\Lambda} \varphi_{0}}{L}+\zeta^{B}\right|^{2}-A^{2}\right)^{2}} F,
$$

where the two cases we are interested in are $F=1$, for $P_{0}$, and

$$
F(\sigma+\tilde{\sigma}, \tilde{\pi})=2 \lambda \gamma w_{\Lambda}\left((A+\sigma+\tilde{\sigma})^{2}+\tilde{\pi}^{2}-A^{2}\right),
$$

for $P_{F}$. At this point we are in a situation similar to that in [GK], and we perform similar manipulations. Actually the analysis here is easier because we are not concerned with a delicate approach to the fixed point, as they were. Some differences arise because the framework we use to keep track of the analytic properties of the remainder is not the same as theirs.

First, write

$$
\begin{aligned}
I= & \frac{1}{N} \int d \tilde{\sigma} d \tilde{\pi} \exp \left\{-\left(\gamma w_{A}\right)^{-1}\left(\tilde{\sigma}^{2}+\tilde{\pi}^{2}\right)-g_{2}(\sigma+\tilde{\sigma})^{2}\right. \\
& \left.-\left[\lambda\left((A+\sigma+\tilde{\sigma})^{2}+\tilde{\pi}^{2}-A^{2}\right)^{2}-g_{2}(\sigma+\tilde{\sigma})^{2}\right]\right\} F(\sigma+\tilde{\sigma}, \tilde{\pi})
\end{aligned}
$$

and then shift

$$
\tilde{\sigma} \rightarrow \tilde{\sigma}-\frac{g_{2}}{\left(\gamma w_{A}\right)^{-1}+g_{2}} \sigma
$$


to find

$$
\begin{gathered}
I=e^{-g_{2} \mathfrak{g} \sigma^{2}-\frac{g_{2}}{A}(\mathfrak{I} \sigma)^{3}-\frac{g_{2}}{4 A^{2}}(\mathfrak{I} \sigma)^{4}} \frac{1}{N} \int d \tilde{\sigma} d \tilde{\pi} \exp \left\{-\left(\gamma w_{A}\right)^{-1}\left(\tilde{\sigma}^{2}+\tilde{\pi}^{2}\right)\right. \\
\left.-\lambda\left((A+\tilde{\sigma})^{2}+\tilde{\pi}^{2}-A^{2}\right)^{2}-\mathrm{v}(\mathfrak{L} \sigma, \tilde{\sigma}, \tilde{\pi})\right\} F(\mathfrak{L} \sigma+\tilde{\sigma}, \tilde{\pi}), \\
\mathrm{v}(\mathfrak{L} \sigma, \tilde{\sigma}, \tilde{\pi})=(\mathfrak{L} \sigma)\left[\frac{3 g_{2}}{A} \tilde{\sigma}^{2}+\frac{g_{2}}{A} \tilde{\pi}^{2}+\frac{g_{2}}{A^{2}} \tilde{\sigma}\left(\tilde{\sigma}^{2}+\tilde{\pi}^{2}\right)\right] \\
+(\mathfrak{L} \sigma)^{2}\left[\frac{3 g_{2}}{A} \tilde{\sigma}+\frac{3 g_{2}}{2 A^{2}} \tilde{\sigma}^{2}+\frac{g_{2}}{2 A^{2}} \tilde{\pi}^{2}\right] \\
+(\mathfrak{L} \sigma)^{3}\left[\frac{g_{2}}{A^{2}} \tilde{\sigma}\right],
\end{gathered}
$$

where we have set

$$
\mathfrak{L} \equiv\left(1+g_{2} \gamma w_{\Lambda}\right)^{-1}
$$

Now define

$$
\chi(\tilde{\sigma}, \tilde{\pi})= \begin{cases}1 & \text { if }|\tilde{\sigma}|,|\tilde{\pi}| \leqq A^{\varepsilon^{\prime}} \\ 0 & \text { otherwise }\end{cases}
$$

with $\varepsilon^{\prime}>\varepsilon$, and insert $1=\chi+\chi^{c}$ inside the integral. We name the resulting terms

$$
P=P_{0}^{\chi}+P_{0}^{c}+P_{F}^{\chi}+P_{F}^{c}
$$

and estimate the $P_{c}$ terms first. We use

$$
|\mathrm{v}(\mathfrak{L} \sigma, \tilde{\sigma}, \tilde{\pi})| \leqq g_{2}\left[\tilde{\sigma}^{2} O\left(h A^{\varepsilon-1}\right)+\tilde{\pi}^{2} O\left(h A^{\varepsilon-1}\right)+O\left(h^{3} A^{3 \varepsilon-1}\right)+\frac{\mathfrak{L} \sigma}{A^{2}} \tilde{\sigma}\left(\tilde{\sigma}^{2}+\tilde{\pi}^{2}\right)\right]
$$

along with

$$
g_{2} \frac{\mathfrak{I} \sigma}{A^{2}} \tilde{\sigma}\left(\tilde{\sigma}^{2}+\tilde{\pi}^{2}\right) \leqq \lambda O\left(h A^{\varepsilon-1}\right)\left((A+\tilde{\sigma})^{2}+\tilde{\pi}^{2}-A^{2}\right)^{2}+g_{2} \tilde{\sigma}^{2} O\left(h A^{\varepsilon-1}\right)
$$

to get

$$
\begin{aligned}
\operatorname{Re}[ & \left.\lambda\left((A+\tilde{\sigma})^{2}+\tilde{\pi}^{2}-A^{2}\right)^{2}+\mathrm{v}(\mathfrak{L} \sigma, \tilde{\sigma}, \tilde{\pi})\right] \\
\geqq & \frac{\lambda}{2}\left((A+\tilde{\sigma})^{2}+\tilde{\pi}^{2}-A^{2}\right)^{2}-O\left(g_{2} h^{3} A^{3 \varepsilon-1}\right) \\
& \quad-\tilde{\sigma}^{2} O\left(g_{2} h A^{\varepsilon-1}\right)-\tilde{\pi}^{2} O\left(g_{2} h A^{\varepsilon-1}\right),
\end{aligned}
$$

where we have used the fact that $h A^{\varepsilon-1} \ll 1$ for $\varepsilon$ small enough. Hence

$$
\begin{aligned}
\left|I^{c}\right| \leqq & e^{-g_{2} \& \operatorname{Re} \sigma^{2}} \frac{1}{N} \int d \tilde{\sigma} d \tilde{\pi} \chi^{c} \exp \left\{-\frac{1}{2}\left(\gamma w_{\Lambda}\right)^{-1}\left(\tilde{\sigma}^{2}+\tilde{\pi}^{2}\right)\right. \\
& \left.\quad-\frac{1}{2} \lambda\left((A+\tilde{\sigma})^{2}+\tilde{\pi}^{2}-A^{2}\right)^{2}-O\left(g_{2} h A^{3 \varepsilon-1}\right)\right\}|F(\mathfrak{L} \sigma+\tilde{\sigma}, \tilde{\pi})| .
\end{aligned}
$$


Now, for $P_{0}$ we have $F=1$, and for $P_{F}$ we have the estimate

$$
|F(\mathfrak{L} \sigma+\tilde{\sigma}, \tilde{\pi})| \leqq \frac{1}{2} g_{2} \gamma w_{\Lambda}\left[O\left(h A^{\varepsilon-1}\right)+\tilde{\sigma} O\left(A^{-1}\right)+\frac{\tilde{\sigma}^{2}+\tilde{\pi}^{2}}{A^{2}}\right],
$$

so in both cases we obtain the bound

$$
\chi^{c} e^{-\frac{1}{2}\left(\gamma w_{1}\right)^{-1}\left(\tilde{\sigma}^{2}+\tilde{\pi}^{2}\right)}|F| \leqq e^{-O\left(A^{2 \varepsilon^{\prime}}\right)} .
$$

The final observation for the $P^{c}$ terms is that

$$
\int d \tilde{\sigma} d \tilde{\pi} e^{-\frac{1}{2} \lambda\left((A+\tilde{\sigma})^{2}+\tilde{\pi}^{2}-A^{2}\right)^{2}} \leqq O\left(\lambda^{-1 / 2}\right)
$$

which is dominated by the $\exp \left(-O\left(A^{2 \varepsilon^{\prime}}\right)\right)$. So we have shown that

$$
\left\{\begin{array}{l}
\left|P_{0}^{c}(\varphi \bar{\varphi}+l)\right| \\
\left|P_{F}^{c}(\varphi \bar{\varphi}+l)\right|
\end{array}\right\} \leqq e^{K-g_{2} \mathfrak{R R e} \sigma^{2}-O\left(A^{2 b^{\prime}}\right)}
$$

for $\varphi$ in the small-field region.

We look at the $Q$ terms next. First, we examine

$$
Q_{0}=\int d \mu_{\gamma w_{A}}\left(\zeta^{B}\right) r\left((A+\sigma+\tilde{\sigma})^{2}+\tilde{\pi}^{2}\right)
$$

which we write as

$$
Q_{0}=\frac{1}{N} \int d \tilde{\sigma} d \tilde{\pi} e^{-\left(\gamma w_{\Lambda}\right)^{-1}\left(\tilde{\sigma}^{2}+\tilde{\pi}^{2}\right)-\tilde{x} g_{2}(\sigma+\tilde{\sigma})^{2}} e^{\tilde{\alpha} g_{2}(\sigma+\tilde{\sigma})^{2}} r\left((A+\sigma+\tilde{\sigma})^{2}+\tilde{\pi}^{2}\right),
$$

where the choice of $\tilde{\alpha}$ will be made below. Next perform a shift

which result in

$$
\tilde{\sigma} \rightarrow \tilde{\sigma}-\frac{\tilde{\alpha} g_{2}}{\left(\gamma w_{1}\right)^{-1}+\tilde{\alpha} g_{2}} \sigma,
$$

$$
Q_{0}=e^{-\tilde{\alpha} g_{2} \tilde{\mathfrak{L}} \sigma^{2}} \int d \tilde{\sigma} d \tilde{\pi} e^{-\left(\left(\gamma w_{A}\right)^{-1}+\tilde{\alpha} g_{2}\right) \tilde{\sigma}^{2}-\left(\gamma w_{A}\right)^{-1} \tilde{\pi}^{2}} e^{\tilde{\alpha} g_{2}(\tilde{\mathfrak{L}} \sigma+\tilde{\sigma})^{2}} r\left((A+\tilde{\mathfrak{L}} \sigma+\tilde{\sigma})^{2}+\tilde{\pi}^{2}\right),
$$

where $\tilde{\mathfrak{L}} \equiv\left(1+\gamma w_{\Lambda} \tilde{\alpha} g_{2}\right)^{-1}$. An analogous expression holds for $Q_{F}$. We then insert $1=\chi+\chi^{c}$ into this expression. We investigate the $Q^{\chi}$ terms below, and just get crude bounds on the $Q^{c}$ terms now. For this it is convenient to shift back again and observe that we still have at least one of $|\tilde{\sigma}|,|\tilde{\pi}|>O\left(A^{\varepsilon^{\prime}}\right)$ since the shift is smaller than $O\left(A^{\varepsilon}\right)$ for all small-field values of $\varphi$ and values that $g_{2}$ can take on, and we have chosen $\varepsilon^{\prime}>\varepsilon$. So we can use the bounds on $r$ from Corollary 4.7 again, and find

$$
\left|Q_{0}^{c}\right|,\left|Q_{F}^{c}\right| \leqq e^{K-O\left(A^{2 \varepsilon^{\prime}}\right)}
$$

We now consider the $P^{\chi}, Q^{\chi}$ terms where $\sigma / A, \tilde{\sigma} / A$, and $\tilde{\pi} / A$ are small. First handle $P_{0}$, defining

$$
\begin{aligned}
\langle\cdot\rangle_{t} \equiv & \frac{1}{N} \int d \tilde{\sigma} d \tilde{\pi} \chi(\tilde{\sigma}, \tilde{\pi})(\cdot) e^{\left(\gamma w_{A}\right)^{-1}\left(\tilde{\sigma}^{2}+\tilde{\pi}^{2}\right)-\lambda\left((A+\tilde{\sigma})^{2}+\tilde{\pi}^{2}-A^{2}\right)^{2}-t \mathrm{v}(\mathfrak{L} \sigma, \tilde{\sigma}, \tilde{\pi})} \\
= & \frac{1}{N} \int d \tilde{\sigma} d \tilde{\pi} \chi(\tilde{\sigma}, \tilde{\pi})(\cdot) e^{\left.-\left(\left(\gamma w_{A}\right)^{-1}+g_{2}\right) \tilde{\sigma}^{2}-\left(\gamma w_{A}\right)^{-1} \tilde{\pi}^{2}\right)} \\
& \times e^{-\frac{g_{2}}{A} \tilde{\sigma}\left(\tilde{\sigma}^{2}+\tilde{\pi}^{2}\right)-\frac{g_{2}}{4 A^{2}}\left(\tilde{\sigma}^{2}+\tilde{\pi}^{2}\right)^{2}-t \mathrm{v}(\mathfrak{Q} \sigma, \tilde{\sigma}, \tilde{\pi})}
\end{aligned}
$$


and performing a perturbation expansion:

$$
\begin{aligned}
P_{0}^{\chi} & =e^{-P_{\exp }(\sigma)}\langle 1\rangle_{t=1}, \\
\langle 1\rangle & =\sum_{i=0}^{2} \frac{t^{i}}{i !}\left\langle(-\mathrm{v})^{i}\right\rangle_{0}+\frac{1}{2} \int_{0}^{t} d t(1-t)^{2}\left\langle(-\mathrm{v})^{3}\right\rangle_{t} \\
& =\langle 1\rangle_{0}\left(1+(\mathfrak{L} \sigma) a_{1}+(\mathfrak{L} \sigma)^{2} a_{2}+a_{3}(\sigma)\right) .
\end{aligned}
$$

Here $a_{1}=O\left(g_{2} / A\right)$ and $a_{2}=O\left(g_{2} / A^{2}\right)$ are constants, and $a_{3}(\sigma)$ is a function satisfying $\left.a_{3}^{(n)}\right|_{\sigma=0}=0$ for $0 \leqq n<3$, analytic for $\sigma$ in the region $|\sigma|<2 h A^{\varepsilon}$, and in this region satisfying $\left|a_{3}(\sigma)\right|<O\left(A^{4 \varepsilon-2}\right)$. Also, we have set

$$
P_{\text {exp }}(\sigma) \equiv-K+g_{2} \mathfrak{L} \sigma^{2}+\frac{g_{2}}{A}(\mathfrak{L} \sigma)^{3}+\frac{g_{2}}{4 A^{2}}(\mathfrak{L} \sigma)^{4} .
$$

We do a similar calculation for $P_{F}^{\chi}$, finding

$$
P_{F}^{\chi}=e^{-P_{\text {×xp }}(\sigma)}\langle 1\rangle_{0}\left(k_{0}+(\mathfrak{L} \sigma) k_{1}+(\mathfrak{L} \sigma)^{2} k_{2}+k_{3}(\sigma)\right),
$$

where $k_{0}=O\left(g_{2} / A^{2}\right), k_{1}=O\left(g_{2} / A\right), k_{2}=O\left(g_{2} / A^{2}\right)$ are constants, and $k_{3}(\sigma)$ is a function satisfying $\left.k_{3}^{(n)}\right|_{\sigma=0}=0$ for $0 \leqq n<3$, analytic for $\sigma$ in the region $|\sigma|<2 h A^{\varepsilon}$, and in this region satisfying $\left|k_{3}(\sigma)\right|<O\left(A^{3 \varepsilon-3} / h\right)$.

We next focus on the $Q$ terms. We need only a very crude bound for $Q_{F}^{x}$, which we obtain from Corollary 4.7. We must first verify that the definition of $\sigma$ and the complex shift of $\tilde{\sigma}$ has not taken us out of the region of validity of the Corollary. Recall from (5.26) that in $Q_{F}^{x}$ we are evaluating $r^{\prime}$ at $(A+\mathfrak{\Omega} \sigma+\tilde{\sigma})^{2}+\tilde{\pi}^{2} \equiv|\tilde{\varphi}|^{2}+\tilde{l}$, where we have defined

$$
\begin{aligned}
|\tilde{\varphi}|^{2} & \equiv(A+\operatorname{Re} \tilde{\mathfrak{L}} \sigma+\tilde{\sigma})^{2}+\tilde{\pi}^{2}, \\
\tilde{l} & \equiv 2 i(\operatorname{Im} \tilde{\mathfrak{L}} \sigma)(A+\operatorname{Re} \mathfrak{L} \sigma+\tilde{\sigma})-(\operatorname{Im} \tilde{\mathfrak{L}} \sigma)^{2} .
\end{aligned}
$$

It is not hard to see that, for $\varphi$ in the small-field region, $|\tilde{\eta}| \leqq 20 w_{A} h^{\prime}|\tilde{\varphi}| / L$, and since $h^{\prime} \leqq h$ we can safely apply Corollary 4.7 to find

$$
\begin{aligned}
\left|e^{\tilde{\alpha} g_{2}(\tilde{\Sigma} \sigma+\tilde{\sigma})^{2}} r^{\prime}\left(|\tilde{\varphi}|^{2}+\tilde{l}\right)\right| & \leqq\left|e^{\tilde{\alpha} g_{2}(\operatorname{Re} \tilde{\tilde{I}} \sigma+\tilde{\sigma})^{2}} r^{\prime}\left(|\tilde{\varphi}|^{2}+\tilde{l}\right)\right| \\
& \leqq O\left(\lambda^{1 / 2}\right)|r|_{K, \alpha g_{2}, A, h} e^{K}
\end{aligned}
$$

if $\tilde{\alpha}<\alpha / 2$. From this we immediately have

$$
\left|Q_{F}^{\chi}\right| \leqq O(\lambda) e^{-\tilde{\alpha} g_{2} \tilde{\Sigma} \operatorname{Re} \sigma^{2}}
$$

In order to deal with $Q_{0}^{\chi}$ we define

$$
\begin{aligned}
H\left(|u|^{2}\right) & \equiv\left[h^{-3}(|u|-A)^{3} e^{K-\tilde{\alpha} g_{2}(\operatorname{Re} \tilde{\mathbb{Y}} \sigma+\tilde{\sigma})^{2}}|r|_{K, \alpha g_{2}, A, h}\right]^{-1} r\left(|u|^{2}\right), \\
u & \equiv|\tilde{\varphi}| \quad \bar{u} \equiv|\tilde{\varphi}|+\tilde{l} /|\tilde{\varphi}|, \\
|u| & \equiv(u \bar{u})^{1 / 2} .
\end{aligned}
$$

\section{Lemma 5.9.}

$$
\mid H\left(|u|^{2} \mid \leqq O(1)\right.
$$

for $u, \bar{u}$ as in (5.32) and $\varphi$ in the small-field region. 
Proof. We use two estimates of $r\left(|u|^{2}\right)$. The first follows from (5.9) by bounding $r^{\prime \prime \prime}$ with Corollary 4.7 and using $\left|\left(|u|^{2}-A^{2}\right)\right| \leqq O(A)|(|u|-A)|$ to find

$$
\left|r\left(|u|^{2}\right)\right| \leqq O\left(h^{-3}\right) e^{K}|r|_{K, \alpha g_{2}, A, h}\left|(|u|-A)^{3}\right|,
$$

where $O(\cdot)$ does not depend on $L$. Also, a direct application of Corollary 4.7 reveals

$$
\left|r\left(|u|^{2}\right)\right| \leqq O(1)|r|_{K, \alpha g_{2}, A, h} e^{K-\frac{\alpha}{2}(\operatorname{Re} \mathscr{Q} \sigma+\tilde{\sigma})^{2}} .
$$

These two facts together yield

$$
\left|r\left(|u|^{2}\right)\right| \leqq O\left(h^{-3}\right)|r|_{K, \alpha g_{2}, A, h} e^{K-\frac{\alpha}{2}(\operatorname{Re} g+\tilde{\sigma})^{2}}\left|(|u|-A)^{3}\right|
$$

since, for $|\operatorname{Re} \mathscr{Q} \sigma+\tilde{\sigma}| \leqq h$ the right-hand side of (5.36) is larger than that of (5.34), while for $|\operatorname{Re} \mathfrak{Q} \sigma+\tilde{\sigma}|>h$ it is larger than that of (5.35). The result (5.33) follows immediately.

We can now write

$$
Q_{0}^{\chi}=h^{-3}|r|_{K, \alpha g_{2}, A, h} e^{K-\tilde{\alpha} g_{2} \tilde{\Sigma} \sigma^{2}} \frac{1}{N} \int d \tilde{\sigma} d \tilde{\pi} \chi e^{-\left(\left(\gamma w_{A}\right)^{-1}+\tilde{\alpha} g_{2}\right) \tilde{\sigma}^{2}-\left(\gamma w_{A}\right)^{-1} \tilde{\pi}^{2}} H\left(|u|^{2}\right)(|u|-A)^{3} .
$$

Now, it is not hard to see that

$$
(|z|-A)^{3}=(\tilde{\mathfrak{L}} \sigma+\tilde{\sigma})^{3}+O\left(h^{5} A^{5 \varepsilon^{\prime}-2}\right),
$$

and so

$$
Q_{0}^{\chi}=\langle 1\rangle_{Q}|r|_{K, \alpha g_{2}, A, h} e^{K-\tilde{\alpha} g_{2} \tilde{L} \sigma^{2}}\left(\sum_{i=0}^{3} b_{i}(\sigma)(\tilde{\mathfrak{L}} \sigma)^{i}+b_{\mathrm{e}}(\sigma)\right)
$$

with

$$
\langle 1\rangle_{Q} \equiv \frac{1}{N} \int d \tilde{\sigma} d \tilde{\pi} \chi e^{-\left(\left(\gamma w_{A}\right)^{-1}+\tilde{\alpha} g_{2}\right) \tilde{\sigma}^{2}-\left(\gamma w_{A}\right)^{-1} \tilde{\pi}^{2}}
$$

and the $b_{i}$ are functions of $\sigma$ satisfying

$$
\begin{aligned}
&\left|b_{i}(\sigma)\right| \leqq O\left(h^{-3}\right)\left(\left(\gamma w_{A}\right)^{-1}+\tilde{\alpha} g_{2}\right)^{\frac{i-3}{2}}, \\
&\left|b_{\mathrm{e}}(\sigma)\right| \leqq O\left(h^{2} A^{5 \varepsilon^{\prime}-2}\right)
\end{aligned}
$$

with $O(\cdot)$ not depending on $L$. The $b_{i}$ and $b_{\mathrm{e}}$ are analytic in $\sigma$ for $|\operatorname{Im} \sigma|<\tilde{\mathfrak{Q}}^{-1} h / 4$, $|\operatorname{Re} \sigma|<2 h A^{\varepsilon}$. Since we are interested in $\sigma$ satisfying $|\operatorname{Im} \sigma|<12 w_{\Lambda} h^{\prime} / L$, we can use Cauchy's estimate to bound derivatives of the $b$ 's:

$$
\begin{aligned}
&\left|\frac{\partial^{j}}{\partial \sigma^{j}} b_{i}(\sigma)\right| \leqq c(j) \tilde{\mathfrak{I}}^{j} h^{-3-j}\left(\left(\gamma w_{A}\right)^{-1}+\tilde{\alpha} g_{2}\right)^{\frac{i-3}{2}}, \\
&\left|\frac{\partial^{j}}{\partial \sigma^{j}} b_{\mathrm{e}}(\sigma)\right| \leqq c(j) \tilde{\mathfrak{Q}}^{j} h^{2-j} A^{5 \varepsilon^{\prime}-2} .
\end{aligned}
$$

We can now assemble the expressions (5.24), (5.27), (5.29), (5.30), (5.31), and (5.38), for the $P$ and $Q$ terms with $\varphi$ in the low-mass region, into a form that allows 
us to find $\tilde{K}, \tilde{\lambda}$, and $\tilde{A}$ and to complete the proof that $\tilde{R}$ satisfies $\tilde{r}$ estimates. We first recall our current status:

$$
\begin{aligned}
\mathscr{R} \mu_{\Gamma W_{A}} *\left(S_{0}+S_{1}\right) & =P^{L^{d}}+L^{d} P^{L^{d}-1} Q+\tilde{r} \\
& \equiv e^{\tilde{K}-\tilde{\lambda}\left(\Phi^{2}-\tilde{A}^{2}\right)^{2}}+\tilde{R}, \\
P & =P_{0}^{c}+P_{F}^{c}+P_{0}^{\chi}+P_{F}^{\chi}, \\
Q & =Q_{0}^{c}+Q_{F}^{c}+Q_{0}^{\chi}+Q_{F}^{\chi} .
\end{aligned}
$$

We are applying Corollary 4.5 to $\tilde{R}$, and we have already shown the necessary analyticity as well as the upper bounds in the large- $\varphi$ region. So all that remains is to show upper bounds in the small- $\varphi$ region and to check the derivative conditions (where we evaluate derivatives at $|\varphi|=A^{\prime}$ ). From the work we have done it is clear that, if we expand the powers of $P$ in (5.40), most of the terms already satisfy the requisite bounds: namely, any term containing at least one $P^{c}$ or $Q^{c}$ term, any term containing more than one $P_{F}^{\chi}$. The delicate choice of $\tilde{K}, \tilde{\lambda}, \tilde{A}$ is then made to compensate for the remaining terms, which are

$$
\begin{gathered}
e^{-L^{4} P_{\exp }(\sigma)}\langle 1\rangle_{0}^{L^{4}}\left[\left(1+(\mathscr{L} \sigma) a_{1}+(\mathscr{L} \sigma)^{2} a_{2}+a_{3}(\sigma)\right)^{L^{4}}\right. \\
\left.+L^{4} e^{P_{\exp }}\langle 1\rangle_{0}^{-1} P_{F}^{\chi}+L^{4} e^{P_{\exp }(\sigma)}\langle 1\rangle_{0}^{-1} Q_{0}^{\chi}\right] ;
\end{gathered}
$$

i.e. the difference of these with $\exp \left(\tilde{K}-\tilde{\lambda}\left(|\varphi|^{2}-\tilde{A}^{2}\right)^{2}\right)$ will satisfy $\tilde{r}^{\prime}$ estimates, where any term $\tilde{r}^{\prime}$ that satisfies

$$
\begin{aligned}
\left|\tilde{r}^{\prime}\right| & \leqq L^{-2} c_{2} \sqrt{\lambda^{\prime}} e^{K^{\prime}-\alpha^{\prime} g_{2}^{\prime}\left(|\varphi|-A^{\prime}\right)^{2}}, \\
\left|\left(\tilde{r}^{\prime}\right)^{(\alpha)}\right|_{\Phi^{2}=A^{\prime 2}} & \leqq O(1) e^{K^{\prime}} \lambda^{1+\frac{\alpha}{2}}
\end{aligned}
$$

is said to satisfy $\tilde{r}^{\prime}$ estimates, or to be an $\tilde{r}^{\prime}$ term. We Taylor expand

$$
\begin{aligned}
\left(1+(\mathfrak{L} \sigma) a_{1}+(\mathfrak{L} \sigma)^{2} a_{2}+a_{3}(\sigma)\right)^{L^{4}} & =1+\tilde{a}_{1} \sigma+\tilde{a}_{2} \sigma^{2}+\tilde{a}_{3}(\sigma), \\
m_{3}(\sigma) \equiv L^{4} e^{P_{\exp }(\sigma)}\langle 1\rangle_{0}^{-1} P_{F}^{\chi} & =M_{0}+M_{1} \sigma+M_{2} \sigma^{2}+M_{3}(\sigma), \\
n_{3}(\sigma) \equiv L^{4} e^{P_{\exp }}\langle 1\rangle_{0}^{-1} Q_{0}^{\chi} & =N_{0}+N_{1} \sigma+N_{2} \sigma^{2}+N_{3}(\sigma),
\end{aligned}
$$

where

$$
\begin{aligned}
M_{3}(\sigma) & =\frac{1}{2} \int_{0}^{1}(1-t)^{2} m_{3}^{\prime \prime \prime}(t \sigma) \sigma^{3}, \\
N_{3}(\sigma) & =\frac{1}{2} \int_{0}^{1}(1-t)^{2} n_{3}^{\prime \prime \prime}(t \sigma) \sigma^{3},
\end{aligned}
$$

and we will argue that $e^{-L^{4} P_{\exp (\sigma)}}\langle 1\rangle_{0}^{L^{4}}\left[\tilde{a}_{3}(\sigma)+M_{3}(\sigma)+N_{3}(\sigma)\right]$ satisfies $\tilde{r}^{\prime}$ estimates.

We use

\section{Lemma 5.10.}

$$
\left|e^{-\kappa L^{4} g_{2} \mathscr{I} \sigma^{2}} \sigma^{m}\right| \leqq c(m) \frac{h^{m}}{(\kappa \mathfrak{L})^{m / 2} L^{2 m}}
$$

for $\kappa=O(1),|\operatorname{Im} \sigma|<12 w_{\Lambda} h^{\prime} / L,|\operatorname{Re} \sigma|<2 h A^{\varepsilon}$. 
Note that the exponential in the Lemma is the leading behavior of $e^{-L^{4} P_{\exp }(\sigma)}$. This tells us essentially that, in estimating the Taylor remainders, each $\sigma$ gives us a factor of $O\left(L^{-2} \mathfrak{Q}^{-1 / 2}\right) h$. Also, from (5.39) we see that each $d / d \sigma$ acting on a $b_{i}$ yields a factor of $O(\tilde{\mathfrak{L}} / h)$. Using these facts it is not hard to see that, for $g_{2} \leqq 1$,

$$
\left|e^{-L^{4} P_{\operatorname{cxp}}(\sigma)}\langle 1\rangle_{0}^{L^{4}} N_{3}(\sigma)\right| \leqq O\left(L^{-2}\right)\langle 1\rangle_{0}^{L^{4}} e^{L^{4} K-\frac{1}{2} L^{4} g_{2} \mathscr{Q R e} \sigma^{2}} \frac{\langle 1\rangle_{Q}}{\langle 1\rangle_{0}}|r|_{K, \alpha g_{2}, A, h},
$$

while for $g_{2}$ near the fixed point value of $O\left(L^{2}\right)$,

$$
\left|e^{-L^{4} P_{\operatorname{cxp}}(\sigma)}\langle 1\rangle_{0}^{L^{4}} N_{3}(\sigma)\right| \leqq O\left(L^{-3}\right)\langle 1\rangle_{0}^{L^{4}} e^{L^{4} K-\frac{1}{2} L^{4} g_{2} \& R e \sigma^{2}} \frac{\langle 1\rangle_{Q}}{\langle 1\rangle_{0}}|r|_{K, \alpha g_{2}, A, h},
$$

(for intermediate values of $g_{2}$ the bound interpolates between these two). We have used half the decay from $e^{L^{4} P_{\exp }(\sigma)}$ in applying Lemma 5.10, and the half that is left is more than enough to cancel the weight function. Now, $\langle 1\rangle_{Q} /\langle 1\rangle_{0}=O(1)$, so we see we have the first of the $\tilde{r}^{\prime}$ estimates if we make the choice

$$
K^{\prime}=L^{4} K+L^{4} \ln \langle 1\rangle_{0}+O(1) .
$$

The second $\tilde{r}^{\prime}$ condition is obvious, using $\left|A^{\prime}-L A / w_{A}\right| \leqq O\left(A^{-1}\right)$. Similar analyses of the $M_{3}, \tilde{a}_{3}$ terms show that they are also $\tilde{r}^{\prime}$. We have hence reduced to

$$
\begin{aligned}
\mathscr{R} \mu_{\Gamma W_{A}} *\left(S_{0}+S_{1}\right)= & e^{-L^{4} P_{\exp }(\sigma)}\langle 1\rangle_{0}^{L^{4}}\left(1+M_{0}+N_{0}\right. \\
& \left.+\left(\tilde{a}_{1}+M_{1}+N_{1}\right) \sigma+\left(\tilde{a}_{2}+M_{2}+N_{2}\right) \sigma^{2}\right)+\tilde{r}^{\prime}+\tilde{r} .
\end{aligned}
$$

We can easily see that the $\left|N_{i}\right| \leqq O\left(\lambda^{1 / 2} h^{-3}\right)$, that $\left|M_{0}\right|,\left|M_{2}\right| \leqq O(\lambda),\left|M_{1}\right|$ $\leqq O\left(g_{2} / A\right)$, and $\left|\tilde{a}_{1}\right| \leqq O\left(g_{2} / A\right),\left|\tilde{a}_{2}\right| \leqq O\left(g_{2} / A^{2}\right)$, where $O(\cdot)$ can depend on $L$. We write $O_{i} \equiv \tilde{a}_{i}+N_{i}+M_{i}$, and

$$
1+O_{0}+O_{1} \sigma+O_{2} \sigma^{2}=\left(1+O_{0}\right)\left(1+C \sigma+\frac{C^{2}}{2} \sigma^{2}\right)\left(1+D \sigma^{2}\right)+O_{\mathrm{r}}
$$

with

$$
\begin{aligned}
C & =\frac{O_{1}}{1+O_{0}}, \\
D & =\frac{O_{2}}{1+O_{0}}-\frac{1}{2}\left(\frac{O_{1}}{1+O_{0}}\right)^{2}, \\
O_{\mathrm{r}} & =-\left(1+O_{0}\right)\left(C D \sigma^{3}+\frac{1}{2} C^{2} D \sigma^{4}\right) .
\end{aligned}
$$

$|C| \leqq O\left(g_{2} / A\right),|D| \leqq O\left(g_{2}^{2} / A\right)$, so clearly $O_{\mathrm{r}}$ is $\tilde{r}^{\prime}$. We can then write

$$
\mathscr{R} \mu_{\Gamma W_{A}} *\left(S_{0}+S_{1}\right)=e^{-L^{4} P_{\operatorname{cxp}}(\sigma)}\langle 1\rangle_{0}^{L^{4}}\left(1+O_{0}\right)\left(e^{C \sigma}-e_{3}^{C \sigma}\right)\left(e^{D \sigma^{2}}-e_{2}^{D \sigma^{2}}\right)+\tilde{r}^{\prime}+\tilde{r}
$$

and, expanding the product, easily see that any term containing an $e_{i}$ is $\tilde{r}^{\prime}\left(e_{i}\right.$ was defined in (5.6)). So we have further reduced to

$$
\begin{aligned}
\mathscr{R} \mu_{\Gamma W_{A}} *\left(S_{0}+S_{1}\right) & =e^{-L^{4} P_{\exp }(\sigma)}\langle 1\rangle_{0}^{L^{4}}\left(1+O_{0}\right) e^{C \sigma+D \sigma^{2}}+\tilde{r}^{\prime}+\tilde{r} \\
& \equiv e^{\tilde{K}-\tilde{\lambda}\left(|\varphi|^{2}-\tilde{A}^{2}\right)^{2}}+\tilde{R}
\end{aligned}
$$


We next write

$$
\begin{aligned}
\tilde{R}= & e^{\tilde{K}-\tilde{\lambda}\left(|\varphi|^{2}+l-\tilde{A}^{2}\right)^{2}}\left[e^{-L^{4} P_{\exp }(\sigma)}\langle 1\rangle_{0}^{L^{4}}\left(1+O_{0}\right) e^{C \sigma+D \sigma^{2}} e^{-\tilde{K}+\tilde{\lambda}\left(|\varphi|^{2}+l-\tilde{A}^{2}\right)^{2}}-1\right] \\
& +\tilde{r}^{\prime}+\tilde{r}
\end{aligned}
$$

and choose $\tilde{K}, \tilde{\lambda}$, and $\tilde{A}$ so that the $\sigma^{0}, \sigma^{1}$, and $\sigma^{2}$ terms in the exponential inside the brackets cancel, and when we subtract 1 we are left with a $\sigma^{3}$ term that we show is $\tilde{r}^{\prime}$. A calculation shows

$$
\begin{aligned}
\tilde{\lambda}= & \lambda w_{\Lambda}^{4}\left(\mathfrak{L}+L^{-4}\left(\frac{C}{2 g_{2} A}-\frac{D}{g_{2}}\right)\right), \\
A^{2}-\left(\frac{w_{\Lambda} \tilde{A}}{L}\right)^{2}= & \frac{-A C}{g_{2}}\left(L^{+} \mathfrak{L}+\frac{C}{2 g_{2} A}-\frac{D}{g_{2}}\right)^{-1}, \\
\tilde{K}= & L^{4} K+L^{4} \ln \langle 1\rangle_{0}+\ln \left(1+O_{0}\right) \\
& +\tilde{\lambda}\left(\frac{L}{w_{\Lambda}}\right)^{4}\left(A^{2}-\left(\frac{w_{A} \tilde{A}}{L}\right)^{2}\right)^{2} .
\end{aligned}
$$

We are left with

$$
\tilde{R}=e^{\tilde{K}-\tilde{\hat{x}}\left(|\varphi|^{2}+l-\tilde{A}^{2}\right)^{2}}\left[e^{E_{3} \sigma^{3}+E_{4} \sigma^{4}}-1\right]
$$

with

$$
\begin{aligned}
E_{3} & \equiv 4 L^{4} A\left(\frac{\tilde{\lambda}}{w_{A}^{4}}-\lambda \mathfrak{I}^{3}\right) \\
& =\frac{L^{4} \mathfrak{Q} g_{2}}{A}\left(1-\mathfrak{I}^{2}+O\left(\frac{g_{2}}{A}\right)\right), \\
E_{4} & \equiv \tilde{\lambda}\left(\frac{L}{w_{A}}\right)^{4}-L^{4} \mathfrak{I}^{4} \lambda \\
& =L^{4} \lambda \mathfrak{I}\left(1-\mathfrak{I}^{3}+O\left(\frac{g_{2}}{A}\right)\right) .
\end{aligned}
$$

Because $1-\mathfrak{2}^{m}=O\left(g_{2}\right)$ for $g_{2}$ small, we can apply Lemma 5.10 to conclude that

$$
\left|e^{-K^{\prime}+\alpha^{\prime} g_{2}^{\prime}\left(|\varphi|-A^{\prime}\right)^{2}} \tilde{R}\left(|\varphi|^{2}+l\right)\right| \leqq O\left(L^{-2}\right) \lambda^{1 / 2} .
$$

The derivative conditions are easily checked, so we have shown that if we choose $c_{2}$ large enough then

$$
\left(\mathscr{R} \mu_{\Gamma W_{\Lambda}} * g^{\mathscr{G}}\right)\left(W_{\Lambda} \Phi\right)=e^{\tilde{K}-\tilde{\lambda}\left(\Phi^{2}-\tilde{A}^{2}\right)^{2}}+\tilde{r},
$$

with the choices $(5.41)$ of $\tilde{K}, \tilde{\lambda}, \tilde{A}$. All that remains is to absorb the $\tilde{r}$ into a true $r$ via shifts in $\widetilde{K}, \tilde{\lambda}, \widetilde{A} \rightarrow K^{\prime}, \lambda^{\prime}, A^{\prime}$, which we accomplish by writing

$$
e^{\left(\tilde{K}-\tilde{\lambda}\left(\Phi^{2}-A^{2}\right)^{2}\right.}+\tilde{r} \equiv e^{\tilde{K}-\tilde{\lambda}\left(\Phi^{2}-\tilde{A}^{2}\right)^{2}}\left(1+f\left(\Phi^{2}\right)\right)
$$

and proceeding as we did following (5.3). The proof of Proposition 5.7 is complete. 


\section{Green's Functions}

In order to calculate the expectation of the density, we must compute some Green's functions of the $\lambda \Phi^{4}$ theory, namely $\left\langle\varphi_{0} \bar{\varphi}_{0}\right\rangle$ and $\left\langle\varphi_{0} \bar{\varphi}_{0} \Phi_{z}^{2}\right\rangle$. As long as 0 and $z$ are in different blocks, the RGT's of the two blocks do not see each other. When $|z-0|=L$ there will be a single RGT that must incorporate both $\varphi_{0} \bar{\varphi}_{0}$ and $\Phi_{z}^{2}$, and from then on the only distinguished point will be 0 .

Since we are interested only in the limit as $\Lambda, z \rightarrow \infty$, we choose $\Lambda$ and $z$ large enough (depending on $\beta_{0}$ ) so that the induction will have reached the large-mass region before 0 and $z$ merge. So in the small and intermediate mass regimes we need only consider the recursion relations for the functions that, before the first RGT, look like $\varphi \bar{\varphi} g_{0}\left(\Phi^{2}\right)$ and $\Phi^{2} g_{0}\left(\Phi^{2}\right)$.

Actually the situation is even simpler than this due to the fact that we have chosen our walks to begin and end at the origin. To see why, we have

Lemma 6.1. If $F$ is a function of a single variable with exponential decay at $\infty$, then (i)

$$
\begin{aligned}
& \mu_{C} *\left(\varphi \bar{\varphi} F\left(\Phi^{2}\right)\right)=F_{1}\left(\Phi^{2}\right)+\varphi \bar{\varphi} F_{2}\left(\Phi^{2}\right), \\
& \mu_{C} *\left(\psi \bar{\psi} F\left(\Phi^{2}\right)\right)=-F_{1}\left(\Phi^{2}\right)+\psi \bar{\psi} F_{2}\left(\Phi^{2}\right), \\
& \mu_{C} *\left(\Phi^{2} F\left(\Phi^{2}\right)\right)=\Phi^{2} F_{2}\left(\Phi^{2}\right),
\end{aligned}
$$

where the functions $F_{1}, F_{2}$ are the same functions in the three equations.

(ii)

$$
\mu_{c} *\left(\Phi^{\#} F\left(\Phi^{2}\right)\right)=\Phi^{\#} F_{3}\left(\Phi^{2}\right)
$$

where $\Phi^{\#}=\varphi, \bar{\varphi}, \psi$, or $\bar{\psi}$, and $F_{3}$ is independent of \#.

Proof. Both parts follow almost immediately from the proof of [BEI] Theorem 4.2.

Part (i) of this lemma tells us that we do not need to consider the $\varphi \bar{\varphi}$ and $\Phi^{2}$ blocks separately, but that we can do them both at once since the convolution will produce the same function $F_{2}$ multiplying the $\varphi \bar{\varphi}$ or $\Phi^{2}$ in the two cases. It will be more convenient for us to handle the $\Phi^{2}$ case to get control of $F_{2}$. Meanwhile, there will be contributions to $F_{1}$ in the $\varphi \bar{\varphi}$ case that do not occur in the $\Phi^{2}$ case. We will not need to keep any control of these until after 0 and $z$ merge, because in the single RGT when this occurs the $F_{1}$ term will be multiplied by a function of the form $\Phi^{2} G\left(\Phi^{2}\right)$, and hence will remain of the form $\Phi^{2} G\left(\Phi^{2}\right)$ under all subsequent RGTs. We will see in Sect. 7 that the contribution of such a function in the calculation of the density vanishes identically. After the RGT in which 0 and $z$ merge there will be new contributions to $F_{1}$ that we will need to keep some control of, but we postpone discussion of this point until Sect. 6.4.

6.1. Low Mass Flow. The upshot of the above discussion is that, for the small-mass region, we need only keep track of functions that can be represented as 
Inductive Assumption $3\left(e^{\beta_{\Phi \Phi^{2}}} g, d_{2}, d_{4}, M\right)$. The function $j=j(\Phi)$ of a field at a single site satisfies

$$
\begin{aligned}
& e^{\beta \Phi^{2}} j(\Phi) g(\Phi)=e^{\beta \Phi^{2}}\left\{\begin{array}{c}
\varphi \bar{\varphi} \\
\text { or } \\
\Phi^{2}
\end{array}\right\}\left(d_{2}+d_{4}\left(\Phi^{2}-2 G(0)\right)\right) g(\Phi)+t_{1}(\Phi)+\left\{\begin{array}{c}
t_{2}(\Phi) \\
\text { or } \\
0
\end{array}\right\}, \\
& t_{1}=\left\{\begin{array}{c}
\varphi \bar{\varphi} \\
\text { or } \\
\Phi^{2}
\end{array}\right\} t_{R}\left(\Phi^{2}\right), \\
&\left.\left(\frac{d}{d t}\right)^{\alpha} t_{1}(t \Phi)\right|_{t=0}=0 \text { for } 0 \leqq \alpha<6 \\
&\left|\Phi^{2} t_{R}\right|_{\sqrt{\lambda}, h} \leqq c_{3} d_{2} \sqrt{\lambda} \\
& d_{4} \leqq c_{3} \lambda d_{2}, \\
& t_{2}=F\left(\Phi^{2}\right) \text { and has exponential decay at } \infty
\end{aligned}
$$

Here $G \equiv G_{M}+a_{M}$.

We are expanding out to fourth order in $\Phi$ so as to keep careful track of the evolution of $d_{2}$, which will be important for determining the power of the logarithmic corrections to scaling. The reason for the factor $\Phi^{2}-2 G(0)$ multiplying $d_{4}$ is that we have chosen to keep track of the non-remainder part in terms of Wickordered monomials and: $\varphi \bar{\varphi} \Phi^{2}:=\varphi \bar{\varphi}\left(\Phi^{2}-2 G(0)\right)$ up to a function of $\Phi^{2}$, which is in $t_{2}$, while: $\Phi^{4}:=\Phi^{2}\left(\Phi^{2}-2 G(0)\right)$.

We write the RGT acting on $j$ as $S(j)$, defined by

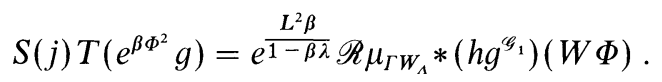

Proposition 6.2. Choose L large enough and $\lambda$ small enough, and suppose $e^{\beta \Phi^{2}} g(\Phi)$ satisfies $I A 1(\beta, \lambda, M)$ and $j(\Phi)$ satisfies $I A 3\left(e^{\beta \Phi^{2}} g, d_{2}, d_{4}, M\right)$. Then $S(h)$ satisfies $\operatorname{IA3}\left(T\left(e^{\beta_{\Lambda} \Phi^{2}} g\right), d_{2}^{\prime}, d_{4}^{\prime}, M-1\right)$ with

$$
\begin{aligned}
& d_{2}^{\prime}=L^{-2} d_{2}\left(1-\gamma_{d} \lambda\right)+O\left(d_{2} \lambda \beta\right), \\
& d_{4}^{\prime}=L^{-4} d_{4}+O\left(d_{2} \lambda\right)
\end{aligned}
$$

where $\gamma_{d}=4 \gamma\left(1-\frac{\gamma}{2}\right)$.

Proof. We will work with the $\Phi^{2}$ case only, since in light of Lemma 6.1 the only additional result we need for the $\varphi \bar{\varphi}$ case is the exponential decay of the $t_{2}$ term, which is obvious. The method established in [BEI] works here as well, so we will not give details but just indicate the origin of the leading contributions. The only differences between our situation and that of [BEI] are that, as mentioned before, our fluctuation covariance $\Gamma W$ is nonsingular and that, due to the presence of $W$, Wick-ordered monomials are no longer exact eigenfunctions of the operator $\mathscr{R} \mu_{\Gamma W^{*}}$. The second one causes some change in the recursion relations which are minor since we stop using this method before $\beta$ becomes large. A calculation of the type (5.1), when applied to the $d_{4}$ term, gives rise to part of the $O\left(\lambda \beta d_{2}\right)$ corrections 
to $d_{2}$. The rest of the $O\left(\lambda \beta d_{2}\right)$ corrections to $d_{2}$ come from a contraction of $d_{2} \Phi_{0}^{2}$ with the $\lambda: \Phi^{4}$ : in the exponent. This term also gives rise to the leading correction to $d_{4}$, which is $O\left(d_{2} \lambda\right)$. The leading contribution to the remainder comes from $d_{2} \Phi_{0}^{2}$ contracting with two copies of $\lambda: \Phi^{4}$ : with two internal legs, yielding a term that is $O\left(d_{2} \lambda^{2}\right) \Phi^{6}$, which is $O\left(\sqrt{\lambda} d_{2}\right)$ in norm.

We next briefly indicate the origin of the $O\left(\lambda d_{2}\right)$ corrections to $d_{2}$. We do this by setting up the tools developed in [BEI], and isolating those terms that contribute to the leading correction to $d_{2}$. The terms we will ignore here contribute to either the corrections to $d_{4}$ or the remainder; we do not go into details involving these last two because the arguments proceed exactly as in [BEI].

We will denote by $s_{I}$ any term that does not contribute to the coefficient of $|\varphi|^{2}$ out to $O\left(d_{2} \lambda\right)$. Begin by writing

$$
S(j) T\left(e^{\beta \Phi^{2}} g\right)=e^{\frac{L^{2} \beta}{1-\beta \gamma} \Phi^{2}} \mathscr{R} E\left(d_{2} \Phi_{0}^{2} g_{0}^{\mathscr{S}_{1}}\right)(W \Phi)+s_{I},
$$

where

$$
\begin{aligned}
E & =1+\Delta_{\Gamma W}+\frac{1}{2} \Delta_{\Gamma W}^{2}, \\
& \doteq \sum_{j=0}^{2} \frac{\left(2 \Delta_{p g}\right)^{j}}{j !} E_{p} E_{g} .
\end{aligned}
$$

Here $\doteq$ denotes equality mod (sixth-order $\partial / \partial \Phi$ derivatives), and the subscripts $p$ and $g$ indicate on which terms the two derivatives in the Laplacians are acting; either $p \equiv d_{2}|\varphi|_{0}^{2}$ or $g^{\mathscr{S}_{1}}$, respectively. Notice that $E_{p} p=p+s_{I}$ since the constant term is $s_{I}$. So we must consider the effects of the three terms involving $\Delta_{p g}$ raised to the powers 0,1 , and 2, and evaluate the results at $W \Phi$. The leading term is

$$
e^{\frac{L^{2} \beta}{1-\beta \gamma} \Phi^{2}} \mathscr{R}\left(p E_{g} g^{\mathscr{G}_{1}}\right)(W \Phi)=d_{2}\left(\frac{w}{L}\right)^{2}\left|\varphi_{0}\right|^{2} T\left(e^{\beta \Phi^{2}} g\right)+s_{I} .
$$

The $\Delta_{p g}$ term yields

$$
\begin{array}{rl}
e^{\frac{L^{2} \beta}{1-\beta \gamma} \Phi^{2}} & \mathscr{R}\left(2 \Delta_{p g} p\left(E_{g} g^{S_{1}}\right)\right)(W \Phi) \\
& =d_{2}\left|\varphi_{0}\right|^{2}(-4 \lambda \gamma w)\left(\left(\frac{w}{L}\right)^{4} 2 G(0)-\left(\frac{w}{L}\right)^{2}(G(0)-\gamma w)\right) T\left(e^{\beta \Phi^{2}} g\right)+s_{I}
\end{array}
$$

with $G \equiv G_{M}+a_{M}$. The $\Delta_{p g}^{2}$ term yields

$$
e^{\frac{L^{2} \beta}{1-\beta \gamma} \Phi^{2}} \mathscr{R}\left(\frac{4 \Delta_{p g}}{2 !} p\left(E_{g} g^{\mathscr{S}_{1}}\right)\right)(W \Phi)=-2 d_{2} \lambda \gamma^{2} \frac{w^{4}}{L^{2}}\left|\varphi_{0}\right|^{2} T\left(e^{\beta \Phi^{2}} g\right)+s_{I} .
$$

Summing these terms gives the result in the statement of the proposition. In particular, we find

$$
\gamma_{d}=4 \gamma\left(1-\frac{\gamma}{2}\right)
$$

We have used the fact that $G(0)=1+O\left(L^{-2 M}\right)$, and $M$ can be taken as large as we like since we are interested in the infinite-volume limit and we apply this proposition in the small-mass region. 
6.2. Switch of Representations. The new representation in the intermediate and large mass regions is

Inductive Assumption $4\left(e^{\beta_{A} \Phi^{2}} g, d_{2}\right)$.

$$
e^{\beta_{\Lambda} \Phi^{2}} j_{\text {new }}(\Phi) g_{\text {new }}(\Phi)=e^{\beta_{A} \Phi^{2}}\left[d_{2}\left\{\begin{array}{c}
\varphi \bar{\varphi} \\
\text { or } \\
\Phi^{2}
\end{array}\right\} g_{\text {new }}(\Phi)+t_{1}^{\text {new }}(\Phi)+\left\{\begin{array}{c}
t_{2}^{\text {new }}(\Phi) \\
\text { or } \\
0
\end{array}\right\}\right]
$$

with

$$
\begin{aligned}
t_{1}^{\text {new }}(\Phi) & =\left\{\begin{array}{c}
\varphi \bar{\varphi} \\
\text { or } \\
\Phi^{2}
\end{array}\right\} t_{R}^{\text {new }}\left(\Phi^{2}\right), \\
\left|t_{1}^{\text {new }}\right|_{\Phi=A} & =0 \\
\left|\Phi^{2} t_{R}^{\text {new }}\right|_{K, \alpha g_{2}, A, h} & \leqq c_{4} \sqrt{\lambda} A^{2} d_{2}, \\
t_{2}^{\text {new }}(\Phi) & =F\left(\Phi^{2}\right), \\
\left|t_{2}^{\text {new }}\right|_{\Phi=0} & \leqq d_{2} e^{K-O\left(A^{2}\right)} \quad \text { after } z \rightarrow 0 \text { only } \\
& \text { and has exponential decay at } \infty .
\end{aligned}
$$

Proposition 6.3. There exists a number $d_{2}^{\text {new }}=d_{2}(1+O(\sqrt{\lambda}))$ and functions $t_{1}^{\text {new }}$, $t_{2}^{\text {new }}$ such that if $e^{\beta_{\Lambda} \Phi^{2}} g_{\text {new }}$ satisfies $I A 2\left(\beta_{\Lambda}, K, \lambda, A, \alpha\right)$ then $j_{\text {new }}=d_{2}^{\text {new }}\{\varphi \bar{\varphi}$ or $\left.\Phi^{2}\right\} g_{\text {new }}+t_{1}^{\text {new }}+t_{2}^{\text {new }}$ satisfies I $A 4\left(e^{\beta_{A} \Phi^{2}} g_{\text {new }}, d_{2}^{\text {new }}\right)$.

Proof. First, write $t_{2}^{\text {new }}=e^{\left(\beta-\beta_{A}\right) \Phi^{2}} t_{2}$. We do not bound $t_{2}^{\text {new }}(0)$ because by assumption $0, z$ are still distinct points at the time of the switch (see comments below Lemma 6.1).

We will prove the $\Phi^{2}$ case first. Then the $\varphi \bar{\varphi}$ case follows immediately. Let $\tilde{d}_{2} \equiv d_{2}-2 G(0) d_{4}$. Then

$$
e^{\beta \Phi^{2}} j(\Phi) g(\Phi)=e^{\beta_{\Lambda} \Phi^{2}}\left\{\tilde{d}_{2} \Phi^{2} g_{\text {new }}\left[1+\frac{d_{4}}{\tilde{d}_{2}} \Phi^{2}+\frac{1}{\tilde{d}_{2} \Phi^{2}} e^{\left(\beta-\beta_{A}\right) \Phi^{2}} t_{1} g_{\text {new }}^{-1}\right]+t_{2}^{\text {new }}\right\} .
$$

Define $f(\Phi)$ by calling the term in the brackets $1+f$. Write $f=f(A)+f \geqq 1$. Then

$$
e^{\beta \Phi^{2}} j(\Phi) g(\Phi)=e^{\beta_{A} \Phi^{2}}\left\{\left[\tilde{d}_{2}(1+f(A)) \Phi^{2} g_{\text {new }}+\tilde{d}_{2} \Phi^{2} g_{\text {new }} f_{\geqq 1}\right]+t_{2}^{\text {new }}\right\} .
$$

We claim the second term is $t_{1}^{\text {new }}$. By its definition it vanishes at $\Phi=A$. That its norm is small enough is easily seen by noting that $|f(A)| \leqq O(\sqrt{\lambda})$, and by applying $|\cdot|_{K, \alpha g_{2}, A, h} \leqq O(1)|\cdot|_{\frac{\sqrt{\lambda}}{3}, \lambda^{-1 / 4}}$ to the various terms one finds in

$$
\left|\tilde{d}_{2} \Phi^{2} g_{\text {new }} f_{\geqq 1}\right|_{K, \alpha g_{2}, A, h} \leqq\left|\tilde{d}_{2} \Phi^{2} g_{\text {new }} f\right|_{K, \alpha g_{2}, A, h}+\left|\tilde{d}_{2} \Phi^{2} g_{\text {new }} f(A)\right|_{K, \alpha g_{2}, A, h}
$$

For example,

$$
\begin{aligned}
d_{4}\left|\Phi^{4} e^{\left(\beta-\beta_{A}\right) \Phi^{2}} g\right|_{K, \alpha g_{2}, A, h} & \leqq O(1) d_{4}\left|\Phi^{4}\right|_{-\frac{\sqrt{\lambda}}{3}, \lambda^{-1 / 4}}\left|e^{\left(\beta-\beta_{A}\right) \Phi^{2}}\right|_{-\frac{\sqrt{\lambda}}{3}, \lambda^{-1 / 4}}|g|_{\sqrt{\lambda}, \lambda^{-1 / 4}} \\
& \leqq O(\lambda) d_{2} O\left(\lambda^{-1}\right) \\
& \leqq c \sqrt{\lambda} A^{2}
\end{aligned}
$$


for $c=c(L)$ chosen large enough. We have used the fact that $A=O\left(\lambda^{-1 / 4}\right)$ at the time of the switch.

We read off $d_{2}^{\text {new }}=\tilde{d}_{2}(1+f(A))$, and we are done.

6.3. Intermediate Mass Flow. From now on we will drop the sub/superscript "new." Within the new representation, we renormalize using the hybrid method of Sect. 5.3 until the radial mass $g_{2}$ becomes larger than $\lambda^{1 / 4+v_{s}}$. The RGT for the new representation is

$$
S(j) T\left(e^{\beta_{\Lambda} \Phi^{2}} g\right)=e^{\frac{L^{2} \beta_{\Lambda}}{1-\beta_{\Lambda} \gamma} \Phi^{2}} \mathscr{R} \mu_{\Gamma W_{\Lambda}} *\left(j g^{\mathscr{G}}\right)\left(W_{\Lambda} \Phi\right) .
$$

Proposition 6.4. Let $g_{2} \in\left[L^{-2} \lambda^{1 / 2} / 5, \lambda^{1 / 4+v_{\delta}}\right], e^{\beta_{\Lambda} \Phi^{2}} g$ satisfying I $A 2\left(\beta_{\Lambda}, K, \lambda, A, \alpha^{(n)}\right)$ and $j$ satisfying $I A 4\left(e^{\beta_{\Lambda} \Phi^{2}} g, d_{2}\right)$. Then $S(j)$ satisfies $I A 4\left(T\left(e^{\beta_{\Lambda} \Phi^{2}} g\right), d_{2}^{\prime}\right)$ with

$$
d_{2}^{\prime}=d_{2}\left(\frac{w_{A}}{L}\right)^{2}\left(1+O\left(\lambda^{1 / 2+2 v_{\delta}}\right)\right) .
$$

Here $n$ is the number of times this Proposition has been applied since the switch of representations, and $\alpha^{(n)}$ was defined in (4.2).

Proof. Again we prove the $\Phi^{2}$ case first, the $\varphi \bar{\varphi}$ case following immediately. We define an approximate remainder term $\tilde{t}_{1}$ for this representation by $\tilde{t}_{1}=\Phi_{0}^{2} F\left(\Phi^{2}\right)$ satisfying

$$
\begin{aligned}
\left|\tilde{t}_{1}\right|_{K^{\prime}, \alpha^{\prime} g_{2}^{\prime}, A^{\prime}, h^{\prime}} \leqq O\left(L^{-4}\right) c_{4} \sqrt{\lambda^{\prime}} A^{\prime 2} d_{2}, \\
\left|\tilde{t}_{1}\right|_{\Phi=A^{\prime}} \leqq O\left(\lambda^{\prime \frac{1}{2}+2 v_{\delta}}\right) A^{\prime 2} e^{K^{\prime}} d_{2} .
\end{aligned}
$$

Later we will show that, with a small shift in $d_{2}, \tilde{t}_{1}$ returns to the inductive form of $t_{1}$.

We begin by writing $\mu_{\Gamma W_{A}{ }^{*}}=1+\Delta+\int d t(1-t) \mu_{t \Gamma W_{A}} * \Delta^{2}$, and show that the Taylor remainder term satisfies $\tilde{t}_{1}$ bounds:

$$
\begin{aligned}
& \left|\mathscr{R} \mu_{t \Gamma W_{A}} * \Delta^{2}\left(j g^{\mathscr{G}_{1}}-t_{2}\right)\left(W_{A} \cdot\right)\right|_{K^{\prime}, \alpha^{\prime} g_{2}^{\prime}, A^{\prime}, h^{\prime}} \\
& \quad=\mid \mu_{t \Gamma W} * \Delta^{2}\left(j g^{\mathscr{G}_{1}}-t_{2}\right) \frac{K_{K^{\prime}}}{L^{4}} \frac{\alpha^{\prime} g_{2}^{\prime}}{L^{2} w_{A}^{2}} \frac{w_{A} A^{\prime}}{L}, \frac{w_{A} h^{\prime}}{L} \\
& \quad \leqq O(1)\left|\Delta^{2}\left(j g^{\mathscr{G}_{1}}\right)\right|_{K, \bar{\alpha} g_{2}, A, \frac{w_{A} h^{\prime}}{L}} \\
& \quad \leqq O\left(h^{-4}\right)\left|\left(j g^{\mathscr{G}}\right)\right|_{K, \bar{\alpha} g_{2}, A, h} \\
& \quad \leqq O\left(h^{-4}\right)\left(\left|d_{2} \Phi_{0}^{2} g\right|_{K, \bar{\alpha} g_{2}, A, h}+\left|t_{1}\right|_{K, \bar{\alpha} g_{2}, A, h}\right)|g|_{K, \bar{\alpha} g_{2}, A, h}^{L^{4}-1} \\
& \left.\quad \leqq O\left(h^{-4}\right)\left(d_{2}\left|\Phi_{0}^{2}\right|_{0,(\bar{\alpha}-\alpha) g_{2}, A, h}|g|_{K, \alpha g_{2}, A, h}\right)+\left|t_{1}\right|_{K, \alpha g_{2}, A, h}\right)|g|_{K, \alpha g_{2}, A, h}^{L^{4}-1} \\
& \quad \leqq c(L) d_{2} A^{2} h^{-4} \\
& \quad \leqq c(L) d_{2} \sqrt{\lambda^{\prime}} A^{\prime 2} \lambda^{\prime 2 v_{\delta}},
\end{aligned}
$$

where we have defined

$$
\bar{\alpha} \equiv\left\{\begin{array}{ll}
\alpha^{\prime}+\frac{2}{3}\left(\alpha-\alpha^{\prime}\right) & \text { if } g_{2}<1 \\
\frac{5}{6} \alpha^{\prime} & \text { if } g_{2} \geqq 1
\end{array} .\right.
$$


We use part of the $\lambda^{2 v_{s}}$ to dominate the $c(L)$, and we have the norm condition for $\tilde{t}_{1}$. The second $\tilde{t}_{1}$ condition follows immediately by bounding the function by its norm.

We have reduced to

$$
S(j) T\left(e^{\beta_{\Lambda} \Phi^{2}} g\right)=e^{\frac{L^{2} \beta_{\Lambda}}{1-\beta_{\lambda} \Phi^{2}}}\left[\mathscr{R}\left(1+\Delta_{\Gamma W}\right)\left(d_{2} \Phi_{0}^{2} g^{\mathscr{S}_{1}}+t_{1} g^{\mathscr{C}_{1} \backslash\{0\}}\right)(W \Phi)+\tilde{t}_{1}+t_{2}\right] .
$$

We claim the term containing $t_{1}$ is $\tilde{t}_{1}$. When the $\Delta$ acts on it, the extra $h^{-2}$ it yields in the norm estimates give us the necessary bounds exactly as above. For the $\mathscr{R} t_{1} g^{\mathscr{G}_{1}} \backslash\{0\}$ term, we appeal to Lemma 5.6 to get the norm condition. The second condition follows by writing

$$
t_{1}(\Phi)=\int_{0}^{1} d t \sum_{|x|=1} \frac{\partial}{\partial \Phi^{\alpha}} t_{1}(t(\Phi-A)+A)(\Phi-A)
$$

and using Lemma 4.1 to bound $\left|\partial_{\Phi} t_{1}\right|$, along with the fact $\left|A^{\prime}-L A / w_{A}\right| \leqq O\left(A^{-1}\right)$.

To handle the leading term, we write

$$
1+\Delta \doteq\left(1+2 \Delta_{p g}\right) E_{p} E_{g},
$$

where $\doteq$ denotes equality up to fourth-order derivatives in $\Phi$ (which are $\tilde{t}_{1}$ terms), and the subscripts $p, g$ indicate whether the derivatives in the Laplacian act on $\Phi_{0}^{2}$ or $g^{\mathscr{S}_{1}}$, respectively. Now, we can replace $E_{p} \Phi_{0}^{2}$ by $\Phi_{0}^{2}$, and $E_{g} g^{\mathscr{S}_{1}}$ by $\mu_{\Gamma W_{A}} * g^{\mathscr{G}_{1}}$ up to $\tilde{t}_{1}$ terms. So the leading term (without the $\Delta_{p g}$ ) yields

$$
d_{2}\left(\frac{w_{A}}{L}\right)^{2} \Phi_{0}^{2} T\left(e^{\beta_{\Lambda} \Phi^{2}} g\right) .
$$

For the other term (with the $\Delta_{p g}$ ) we calculate

$$
E_{g} \frac{\partial}{\partial \Phi_{x}} g^{\mathscr{G}_{1}}=E_{g} \bar{\Phi}_{x}(-2 \lambda)\left(\Phi_{x}^{2}-A^{2}\right) g^{\mathscr{G}_{1}}+\tilde{t}_{1} .
$$

The derivatives in $E_{g}$ can both act on the polynomial, or both on $g^{\mathscr{S}_{1}}$, or one on each. In the last case, we get an extra power of $\lambda$ from the exponent, and the result is a $\tilde{t}_{1}$ term. When they both act on the polynomial, we get $\bar{\Phi}\left(\Phi^{2}-A^{2}\right)+\bar{\Phi}$, and the last term $(\bar{\Phi})$ is $\tilde{t}_{1}$. When they both act on $g^{\mathscr{G}_{1}}$ we get $\mu * g^{\mathscr{G}_{1}}$. So the result of the $\Delta_{p g}$ term is

$$
2 d_{2}(-2 \lambda) L^{4}\left(\frac{w_{A}}{L}\right)^{2} \Phi_{0}^{2}\left(\frac{w_{\Lambda}^{2} \Phi^{2}}{L^{2}}-A^{2}\right) T\left(e^{\beta_{\Lambda} \Phi^{2}} g\right) .
$$

Finally, using $\left|A^{\prime 2}-L^{2} A^{2} / w_{A}^{2}\right| \leqq O(1)$, we can put most of this term in $t_{1}$ at the cost of an $O(\lambda)$ shift in $d_{2}^{\prime}$. We have found

$$
S(j) T\left(e^{\beta_{\Lambda} \Phi^{2}} g\right)=d_{2}\left(\frac{w_{A}}{L}\right)^{2}(1+O(\lambda)) \Phi_{0}^{2} T\left(e^{\beta_{\Lambda} \Phi^{2}} g\right)+e^{\frac{L^{2} \beta_{\Lambda}}{1-\beta_{\lambda} \phi^{2}}}\left[\tilde{t}_{1}+t_{2}\right] .
$$

The final step is to change the $\tilde{t}_{1}$ into a true remainder $t_{1}$ via a small shift in $d_{2}$. To accomplish this we add and subtract on the RHS the term

$$
\Phi_{0}^{2} T\left(e^{\beta_{1} \Phi^{2}} g\right) \tilde{t}_{1}\left(\Phi^{2}=A^{\prime 2}\right) A^{\prime-2} e^{-K^{\prime}},
$$


and claim that the difference of $\tilde{t}_{1}$ and this term becomes the new remainder $t_{1}$. It vanishes at the minimum of the potential by its definition. That its norm is small enough is easy to see, since the norm of (6.3) is smaller than $c(L) A^{2} \lambda d_{2}$, and, due to the $L^{-2}$ contraction we required to be in $\tilde{t}_{1}$, we have plenty of room to add this small correction. The shift in $d_{2}$ is $O\left(\lambda^{\frac{1}{2}+2 v_{\delta}} d_{2}\right)$.

6.4. Large Mass Flow. To handle the induction in the large-mass region, we will need more precise measure of the function $F_{2}$ in Lemma 6.1. This is contained in

\section{Lemma 6.5.}

$$
\mu_{C} *\left(\Phi^{2} F\left(\Phi^{2}\right)\right)=\Phi^{2} \mu_{C} *\left(F+2 C F^{\prime}\right)+C^{2} \mu_{C} *\left(\Phi^{2} F^{\prime \prime}\right)+2 \Phi C^{2} \mu_{C} *\left(\bar{\Phi} F^{\prime \prime}\right) .
$$

Proof. This follows from standard manipulations of Gaussian integrals; see e.g. [BEI] for a review of Gaussian integrals involving Grassman variables. We have used part (ii) of Lemma 6.1 to obtain the last term on the right-hand side.

Proposition 6.6. Let $g_{2}>\lambda^{1 / 4+v_{\delta}}$, and suppose $\exp \left(\beta_{\Lambda} \Phi^{2}\right) g$ satisfies $I A 2\left(\beta_{\Lambda}, K, \lambda, A, \alpha^{(n)}\right)$, and $j$ satisfies $I A 4\left(e^{\beta_{\Lambda} \Phi^{2}} g, d_{2}\right)$. Then $S(j)$ satisfies $\operatorname{IA4}\left(T\left(e^{\beta_{\Lambda} \Phi^{2}} g\right), d_{2}^{\prime}\right)$ with

$$
\begin{aligned}
d_{2}^{\prime} & =d_{2}\left(\frac{w_{A}}{L}\right)^{2}\left(1+O\left(\frac{g_{2}}{A}\right)\right), \\
t_{2}^{\prime}(0) & =t_{2}(0)+d_{2} e^{K-o\left(A^{2}\right)} .
\end{aligned}
$$

Proof. As before, the $\varphi \bar{\varphi}$ case follows from the $\Phi^{2}$ case, with an additional argument to control the $t_{2}(0)$ condition after $z \rightarrow 0$. We will comment on this after we have established the Proposition for the $\Phi^{2}$ case.

In light of Lemma 6.5, the single integral involving fields at the origin becomes

$$
\begin{aligned}
\mathscr{R} \mu_{\gamma w_{A}} * \Phi^{2}\left(d_{2} g+t_{R}\right)\left(w_{\Lambda} \Phi\right)= & \frac{w_{\Lambda}^{2} \Phi^{2}}{L^{2}} \mathscr{R} \mu_{\gamma w_{A}} * H\left(w_{\Lambda} \Phi\right) \\
& +\left(\gamma w_{\Lambda}\right)^{2} \mathscr{R} \mu_{\gamma w_{\Lambda}} *\left(d_{2} g^{\prime \prime}+t_{R}^{\prime \prime}\right)\left(w_{\Lambda} \Phi\right) \\
& +2\left(\gamma w_{\Lambda}\right)^{2} \frac{w_{\Lambda}}{L} \Phi \mathscr{R} \mu_{\gamma w_{\Lambda}} * \bar{\Phi}\left(d_{2} g^{\prime \prime}+t_{R}^{\prime \prime}\right)\left(w_{\Lambda} \Phi\right), \\
H(\Phi) \equiv d_{2}\left(1-4 \gamma w_{\Lambda} \lambda X\right) e^{K-\lambda X^{2}} & +d_{2}\left(r+2\left(\gamma w_{\Lambda}\right) r^{\prime}\right)+t_{R}+2\left(\gamma w_{\Lambda}\right) t_{R}^{\prime},
\end{aligned}
$$

where we have written $X \equiv \Phi^{2}-A^{2}$.

Our strategy will be to find $\bar{d}_{2}$ such that

$$
S(j) T\left(e^{\beta_{\Lambda} \Phi^{2}} g\right)=\bar{d}_{2} \Phi_{0}^{2} T\left(e^{\beta_{\Lambda} \Phi^{2}} g\right)+e^{\frac{L^{2} \beta_{\Lambda}}{1-\beta_{\Lambda} \gamma} \Phi^{2}}\left(\tilde{t}_{1}+t_{2}^{\prime}\right),
$$

where we define the approximate remainders by $\tilde{t}_{1}=\Phi^{2} F\left(\Phi^{2}\right)$ satisfying

$$
\begin{gathered}
\left|\tilde{t}_{1}\right|_{K^{\prime}, \alpha^{\prime} g_{2}^{\prime}, A^{\prime}, h^{\prime}} \leqq O\left(L^{-4}\right) c_{4} \sqrt{\lambda^{\prime}} A^{\prime 2} d_{2} \\
\left|\tilde{t}_{1}\right|_{\Phi=A^{\prime}} \leqq O\left(\lambda^{\prime}\right) A^{\prime 2} e^{K^{\prime}} d_{2} .
\end{gathered}
$$

$\tilde{t}_{1}$ will eventually be absorbed into a small shift in $\bar{d}_{2}$ plus a true remainder term by the same procedure as before. 
Our first observation is that if we expand the product $\left(g_{0}+r\right)^{\mathscr{G}_{1} \backslash\{0\}}$, the terms with more than one $r$ are $\tilde{t}_{1}$, as is easily shown using arguments like (6.2). It is convenient to put most of these terms in $\tilde{t}_{1}$ now so that the factor multiplying (6.4) has good decay properties (we only know that $r$ by itself decays as fast as the norm, while $g_{0}$ decays a good deal faster, a fact that will prove useful in the sequel). So we define

$$
\bar{g} \equiv \sum_{\substack{X \in \mathscr{G}_{1}, X \neq 0 \\|X|=0,1}} r^{X} g_{0}^{\mathscr{G}_{1} \backslash X}
$$

We next argue that the terms containing $g^{\prime \prime}$ or $t_{R}^{\prime \prime}$ are $\tilde{t}_{1}$. That they are of the form $\Phi^{2} F\left(\Phi^{2}\right)$ follows from Lemma 6.1, and we establish the norm condition, which will imply the second condition, as follows. For e.g. the second (and more complicated) term, we set $F \equiv d_{2} g+t_{R}$, and apply Lemma 4.3:

$$
\begin{aligned}
\mid \mathscr{R} & \left.\Phi_{0} \mu_{\Gamma W_{A}} *\left(2 \bar{g}\left(\gamma w_{A}\right)^{2} \bar{\Phi}_{0} F^{\prime \prime}\right)\left(W_{A} \cdot\right)\right|_{K^{\prime}, \alpha^{\prime} g_{2}^{\prime}, A^{\prime}, h^{\prime}} \\
& \leqq O(1) \mid \mathscr{R} \Phi_{0} \mu_{\Gamma W_{A}} *\left(e^{\frac{\lambda}{2}\left(\Phi_{\mathrm{v}}^{2}-A^{2}\right)^{2}} \bar{g} e^{-\frac{\lambda}{2}\left(\Phi_{0}^{2}-A^{2}\right)^{2}} \bar{\Phi}_{0} F^{\prime \prime}\right)(\cdot) \frac{\left.\right|_{K^{\prime}}}{L^{4}}, \frac{\alpha^{\prime} g_{2}^{\prime}}{L^{2} w^{2}}, \frac{w_{A} A^{\prime}}{L}, \frac{w_{A} h^{\prime}}{L} \\
& \leqq c(L)\left|\Phi_{0}\right|_{0,-\left(\bar{\alpha}-\alpha^{\prime}\right) \frac{g_{2}^{\prime}}{L^{2} w_{A}^{2}}(1+c \lambda), A, h}\left|\mu_{\gamma w_{A}} * e^{-\frac{\lambda}{2}\left(\Phi_{0}^{2}-A^{2}\right)^{2}} \bar{\Phi}_{0} F^{\prime \prime}\right|_{K, \frac{\bar{\alpha} g_{2}^{\prime}}{L^{2} w^{2}}(1+c \lambda), A, \frac{w_{A} h^{\prime}}{L}} \\
& \leqq c(L) A\left|e^{-\frac{\lambda}{2}\left(\Phi_{0}^{2}-A^{2}\right)^{2}} \bar{\Phi}_{0} F^{\prime \prime}\right|_{K, \alpha g_{2}, A}, \frac{w_{A} h^{\prime}}{L} \\
& \leqq c(L) d_{2} \lambda A^{2} .
\end{aligned}
$$

We have taken $x \in \mathscr{G}_{1}$ to be a point where $\bar{g}$ does not contain a remainder, and defined

$$
\bar{\alpha}= \begin{cases}\alpha^{\prime}+\frac{1}{3}\left(\alpha-\alpha^{\prime}\right) & \text { if } g_{2} \leqq 1 \\ \frac{7}{6} \alpha^{\prime} & \text { if } g_{2}>1\end{cases}
$$

In the last line we bounded $F^{\prime \prime}$ using Corollary 4.7 and estimated the norm using Corollary 4.5. Now combine (6.4), (6.4), and (6.5); we see that we must show that there is a $\bar{d}_{2}$ such that

$$
\left(\mathscr{R} \mu_{\Gamma W_{A}} * \bar{g}\right)\left(W_{\Lambda} \Phi\right)\left[\frac{w_{\Lambda}^{2}}{L^{2}} \Phi_{0}^{2}\left(\mathscr{R} \mu_{\gamma w_{A}} * H^{\prime}\right)\left(W_{\Lambda} \Phi\right)\right]
$$

satisfies $\tilde{t}_{1}$ bounds, where

$$
\begin{aligned}
H^{\prime}(\Phi) \equiv & H-L^{2} \bar{d}_{2} g / w_{\Lambda}^{2} \\
= & \left(q_{0}+q_{1} X\right) e^{K-\lambda X^{2}}+\left(d_{2}-\frac{L^{2}}{w_{A}^{2}} \bar{d}_{2}\right) r+2 d_{2}\left(\gamma w_{\Lambda}\right) r^{\prime} \\
& +t_{R}+2\left(\gamma w_{\Lambda}\right) t_{R}^{\prime} \\
q_{0}= & d_{2}-\frac{L^{2}}{w_{\Lambda}^{2}} \bar{d}_{2} \\
q_{1}= & -4 \gamma w_{\Lambda} \lambda d_{2} .
\end{aligned}
$$


We now show that, for an appropriate choice of $\bar{d}_{2},(6.6)$ is $\tilde{t}_{1}$. We assume, to be justified later, that $\left|d_{2}-L^{2} \bar{d}_{2} / w_{A}^{2}\right| \leqq O\left(d_{2} g_{2} / A\right)$. We will now proceed as we did in Sect. 5.4, that is, we appeal to general arguments (as was done under (5.14)) to show that (6.6) is analytic in $R_{2 h^{\prime}}$, and so our problem is reduced to bounding (6.6) when evaluated at $\varphi \bar{\varphi}=\left|\varphi^{\prime}\right|^{2}+l$, for $\varphi \in \mathbb{C}$ and $l \in C_{2 h^{\prime}}\left(\varphi^{\prime}\right)$. We again split into two regions of $\left|\varphi^{\prime}\right|^{2}$, the large- and small-field regions, and remark that the large-field estimates go largely as in Sect. 5.4, so we do not repeat those arguments here. In the small-field region, we use the same definitions (5.20), (5.21), and again split into the small- and large- $\zeta^{B}$ regions with (5.23). The large- $\zeta^{B}$ region goes as before, so we will concentrate on the small-field, small- $\zeta^{B}$ region.

Using Corollary 4.5 along with Corollary 4.7 we can relegate most of (6.6) to $\tilde{t}_{1}$. For example, for the term containing $t_{R}^{\prime}$, we get a factor of $\sqrt{\lambda} A^{-2}\left|t_{1}\right|_{K, \alpha q_{2}, A, h} e^{K}$ from Corollary 4.7 and a factor of $O\left(A^{2}\right)$ from the $\Phi^{2}$ in front, with more than enough decay coming from the at least $\left(L^{4}-2\right)$ factors of $g_{0}$ in $\bar{g}$. Hence we find

$$
\left|\frac{w_{\Lambda}^{2} \Phi_{0}^{2}}{L^{2}} \mathscr{R} \mu_{\Gamma W_{A}} *\left(2 \gamma w_{\Lambda} \bar{g} t_{R}^{\prime}\right)\left(W_{A} \cdot\right)\right|_{K^{\prime}, \alpha^{\prime} g^{\prime}, A^{\prime}, h^{\prime}} \leqq O(\sqrt{\lambda})\left|t_{1}\right|_{K, \alpha g_{2}, A, h} .
$$

Similar arguments can be applied to the terms containing $r^{\prime}$ and $\left(d_{2}-L^{2} \bar{d}_{2} /\right.$ $\left.w_{\Lambda}^{2}\right) r$, so we are left with

$$
H^{\prime}(\Phi)=\left(q_{0}+q_{1} X\right) e^{K-\lambda X^{2}}+t_{R}
$$

up to $\tilde{t}_{1}$ terms.

Since we know that (6.6) satisfies the analyticity conditions that Corollary 4.5 require, we can split it into a sum of pieces that individually need not satisfy these conditions and just bound each piece separately. We will call a term $\tilde{t}_{1}^{\prime}$ if it can be written as $\tilde{t}_{1}^{\prime}=\Phi^{2} F\left(\Phi^{2}\right)$ and satisfies

$$
\begin{aligned}
& \left|\left(\left|\varphi^{\prime}\right|^{2}+l\right) F\left(\left|\varphi^{\prime}\right|^{2}+l^{\prime}\right)\right|\left(w^{\prime}\left(\varphi^{\prime}\right)\right)^{-1} \leqq O\left(L^{-4}\right) c_{4} \sqrt{\lambda^{\prime}} A^{\prime 2} d_{2} \\
& \text { for } \varphi^{\prime} \in \mathbb{C} \text { and } l, l^{\prime} \in C_{2 h^{\prime}}\left(\varphi^{\prime}\right) \\
& \left|\tilde{t}_{1}\right|_{\Phi=A^{\prime}} \leqq O\left(\lambda^{\prime}\right) A^{\prime 2} d_{2} e^{K^{\prime}} .
\end{aligned}
$$

We next argue that the $t_{R}$ term is $\tilde{t}_{1}^{\prime}$. We accomplish this by doing the Fermionic integral and observing that the $t_{R}^{\prime}$ term is clearly $\tilde{t}_{1}^{\prime}$. We name the remaining term

$$
T_{0} \equiv \int d \mu_{\gamma w_{A}}\left(\zeta^{B}\right) t_{R}\left(\left|\frac{w_{A} \varphi}{L}+\zeta^{B}\right|^{2}\right) .
$$

We follow the same procedure as below (5.25) and find the only term not manifestly $\tilde{t}_{1}^{\prime}$ is

$$
\begin{aligned}
T_{0}^{\chi}= & \frac{\left|t_{1}\right|_{K, \alpha g_{2}, A, h}}{A^{2} h} e^{K-\tilde{\alpha} g_{2} \tilde{2} \sigma^{2}} \\
& \times \frac{1}{N} \int d \tilde{\sigma} d \tilde{\pi} \chi e^{-\left(\left(\gamma w_{A}\right)^{-1}+\tilde{\alpha} g_{2}\right) \tilde{\sigma}^{2}-\left(\gamma w_{A}\right)^{-1} \tilde{\pi}^{2}} J\left(|u|^{2}\right)(|u|-A),
\end{aligned}
$$

where we have retained the definition (5.32) of $u, \bar{u}$, and defined

$$
J\left(|u|^{2}\right) \equiv\left[h^{-1} A^{-2}(|u|-A) e^{K-\tilde{x} g_{2}(\operatorname{Re} \tilde{Z} \sigma+\tilde{\sigma})^{2}}\left|t_{1}\right|_{K, \alpha g_{2}, A, h}\right]^{-1} t_{R}\left(|u|^{2}\right) .
$$


$|J| \leqq O(1)$ by an argument exactly analogous to Lemma 5.9. Notice that (6.7) is analogous to (5.37), and we proceed in same manner:

$$
\begin{aligned}
& T_{0}^{\chi}=A^{-2}\left|t_{1}\right|_{K, \alpha g_{2}, A, h} e^{K-\tilde{\alpha} g_{2} \tilde{\mathfrak{L}} \sigma^{2}}\langle 1\rangle_{Q}\left(s_{0}(\sigma)+s_{1}(\sigma)(\tilde{\mathfrak{L}} \sigma)+s_{\mathrm{e}}(\sigma)\right), \\
& \left|S_{i}\right| \leqq \frac{O\left(h^{-1}\right)}{\left(\left(\gamma w_{A}\right)^{-1}+\tilde{\alpha} g_{2}\right)^{\frac{1-i}{2}}}, \\
& \left|s_{\mathrm{e}}\right| \leqq O\left(h^{2} A^{3 \varepsilon^{\prime}-2}\right) .
\end{aligned}
$$

Here $s_{i}, s_{\mathrm{e}}$ are analytic for $\sigma$ in the region $|\operatorname{Im} \sigma|<h(4 \tilde{\mathfrak{L}})^{-1},|\operatorname{Re} \sigma|<2 h A^{\varepsilon}$. The term containing the $s_{\mathrm{e}}$ is clearly $\tilde{t}_{1}^{\prime}$. We also write $s_{0}(\sigma)+s_{1}(\sigma) \equiv s_{0}(0)+s_{\mathrm{r}}(\sigma)$, and an application of Lemma 5.10 shows that $s_{r}$ is also $\tilde{t}_{1}^{\prime}$. So the only piece not shown to be $\tilde{t}_{1}^{\prime}$ is the constant term which satisfies the bound

$$
\left.\left|A^{-1}\right| t_{1}\right|_{K, \alpha g_{2}, A, h}\langle 1\rangle_{Q} s_{0}(0) \mid \leqq \frac{\langle 1\rangle_{Q} c_{4} d_{2}}{\left(\left(\gamma w_{A}\right)^{-1}+\tilde{\alpha} g_{2}\right)^{1 / 2}} O\left(\frac{g_{2}}{A}\right) .
$$

We next consider the contribution to (6.6) from the term containing $q_{0}+q_{1} X$. We integrated out the fermions in the fluctuation convolution and obtain

$$
\begin{aligned}
\left.\mathscr{R} \mu_{\gamma w_{A}} *\left(q_{0}+q_{1} X\right) e^{K-\lambda X^{2}}\right|_{\psi=\Psi}=0 & =\int d \mu_{\gamma w_{A}}\left(\zeta^{B}\right) e^{K-\lambda Y^{2}}\left(e_{0}+e_{1} Y\right)+\tilde{t}_{1}^{\prime}, \\
e_{0} & \equiv d_{2}-L^{2} w_{\Lambda}^{-2} \bar{d}_{2}, \\
e_{1} & \equiv-4 \gamma w_{\Lambda} \lambda d_{2}+2 \lambda \gamma w_{\Lambda}\left(d_{2}-L^{2} w_{\Lambda}^{-2} \bar{d}_{2}\right),
\end{aligned}
$$

where on the RHS we have set $Y \equiv\left|\varphi / L+\zeta^{B}\right|^{2}-A^{2}$. We deal with this as we did in Sect. 5.4, by performing a perturbation expansion. Here we only need carry it out to zeroth order, the first correction being $\tilde{t}_{1}^{\prime}$ already. So, recalling the definition (5.28), we write

$$
\begin{aligned}
\int d \mu_{\gamma w_{A}}\left(\zeta^{B}\right) e^{K-\lambda Y^{2}}\left(e_{0}+e_{1} Y\right) & =e^{-g_{2} \mathfrak{I} \sigma^{2}}\langle C\rangle_{0}+\tilde{t}_{1}^{\prime}, \\
C & \equiv e_{0}+e_{1}\left((A+\mathfrak{L} \sigma+\tilde{\sigma})^{2}+\tilde{\pi}-A^{2}\right) .
\end{aligned}
$$

Of the term containing $C$, the pieces containing $\sigma$ and $\sigma^{2}$ are clearly $\tilde{t}_{1}^{\prime}$, assuming we choose $c_{4}$ large enough. Also, since $\langle\tilde{\sigma}\rangle_{0}=O\left(g_{2} / A\right)$, all terms containing an $e_{1}$ are $\tilde{t}_{1}^{\prime}$ as well. So we are left with

$$
\begin{aligned}
(6.6)= & \left(\mathscr{R} \mu_{\Gamma W_{A}} * \bar{g}\right)\left(W_{\Lambda} \Phi\right) \frac{w_{A}^{2}}{L^{2}} \Phi_{0}^{2} \\
& \times\left(e^{-\tilde{\alpha} g_{2} \tilde{g}_{\sigma}^{2}} s_{0}(0)\langle 1\rangle_{Q} A^{-2}\left|t_{1}\right|_{K, \alpha g_{2}, A, h}+e^{-g_{2} \mathfrak{g} \sigma^{2}}\left(d_{2}-\frac{L^{2}}{w_{A}^{2}} \bar{d}_{2}\right)\langle 1\rangle_{0}\right)+\tilde{t}_{1}^{\prime} .
\end{aligned}
$$

We now make the choice

$$
d_{2}-\frac{L^{2}}{w_{\Lambda}^{2}} \bar{d}_{2}=-s_{0}(0) \frac{\langle 1\rangle_{Q}}{\langle 1\rangle_{0}} A^{-2}\left|t_{1}\right|_{K, \alpha g_{2}, A, h}
$$

and then (6.9) becomes $\tilde{t}_{1}^{\prime}$, as is easy to see by expanding the difference in exponentials in a Taylor series to zeroth order and applying Lemma 5.10 to the remainder. 
Since we know that the sum of the $\tilde{t}_{1}^{\prime}$ terms satisfy the necessary analyticity conditions to apply Corollary 4.5 , we see that their sum is actually $\tilde{t}_{1}$. All that remains is to change the $\tilde{t}_{1}$ into a true remainder $t_{1}$ via small shift in $\bar{d}_{2}$. This is accomplished by the procedure described at the end of Sect. 6.3, and incurs a shift of $O(\lambda) d_{2}$, which is smaller than the leading term from (6.10) and (6.8).

The only part of Proposition 6.6 we have not yet proven is the statement involving the $t_{2}$ terms, which occur in the $\varphi \bar{\varphi}$ case. $t_{2}^{\prime}$ has contributions from the image of $t_{2}$ under the RG map and also from contractions of $\varphi$ with $\bar{\varphi}$. Since we are only interested in $t_{2}^{\prime}$ evaluated at zero, we can write

$$
t_{2}^{\prime}(0)=\mathscr{R} \mu_{\Gamma W_{A}} *\left(d_{2} \varphi_{0} \bar{\varphi}_{0} g+t_{1}+t_{2}\right)(0)
$$

because, referring to Lemma 6.1 , the $F_{2}$ terms are multiplied by zero. From [BEI] Theorem 4.2 we see that

$$
\mathscr{R} \mu_{\Gamma W_{A}} * t_{2} g^{\mathscr{G} \backslash\{0\}}(0)=t_{2}(0) .
$$

We then apply Corollary 4.7 to $t_{1}$, and, using estimates similar to (5.17), we see the corrections to $t_{2}(0)$ are smaller than $d_{2} \exp \left(K-O\left(A^{2}\right)\right)$. Finally, notice that $K_{n}-O\left(A_{n}^{2}\right)$, where $n$ is the induction step, is roughly exponentially increasing in $n$. Hence, if $\left|t_{2}(0)\right| \leqq d_{2} \exp \left(K-O\left(A^{2}\right)\right)$ at any given step, it will satisfy the same bound for all remaining steps, which is the fact we need to satisfy $I A 4$. In fact, immediately after 0 and $z$ merge, $t_{2}(0)=0$, so this estimate will hold.

At some point there will be a unique step in which $0, z$ are in the same block. We have assumed that $z$ is large enough (depending on the initial $\beta$ ) that this occurs while we are in the large-mass regime. This one step requires special treatment, but since the proof is so similar to that of Proposition 6.6 we will state without proof.

Proposition 6.7. Suppose $0, z$ are distinct points in $\mathscr{G}_{1}$. Let $g_{2}>\lambda^{1 / 4+v_{\delta}}$, and suppose $\exp \left(\beta_{\Lambda} \Phi^{2}\right) g$ satisfies $I A 2\left(\beta_{\Lambda}, K, \lambda, A, \alpha^{(n)}\right)$, and $j_{0}, j_{z}$ satisfy I $A 4\left(e^{\beta_{\Lambda} \Phi^{2}} g, d_{2}\right)$, with the choice of $\varphi_{0} \bar{\varphi}_{0}$ in $j_{0}$ and $\Phi_{z}^{2}$ in $j_{z}$. Then $S\left(j_{0} j_{z}\right)$ satisfies $I A 4\left(T\left(e^{\beta_{A} \Phi^{2}} g\right), d_{2}^{\prime}\right)$ with the choice of $\varphi_{0} \bar{\varphi}_{0}$, and

$$
\begin{aligned}
d_{2}^{\prime} & =d_{2}^{2} A^{2}\left(\frac{w_{A}}{L}\right)^{2}\left(1+O\left(\frac{g_{2}}{A}\right)\right), \\
t_{2}^{\prime}(0) & =0 .
\end{aligned}
$$

\section{Calculation of Critical Exponents}

In this section we piece together the recursion relations we have determined for the various mass regimes into a calculation of $E_{\lambda, N}\left(\tau_{z}\right)$. We take the infinite volume limit, followed by the limit of $z \rightarrow \infty$, and find the critical exponent for the density.

We choose some $\beta>0$. We will do RGTs using the different methods to keep track of the integrands as $\beta$ grows and the volume shrinks. For convenience assume $\beta<O\left(\lambda^{2}\right)$; this ensures that we start by using the version of the RGT corresponding to very small mass (we can clearly construct the model for other $\beta$ but we are interested just in the asymptotic behavior as $\beta \rightarrow 0$ ). Next choose some $\Lambda$ and $z$ large enough (depending on $\beta$ ) to ensure that we can do enough RGTs to get to 
the large-mass region before the points 0 and $z$ collapse to the same point. Again this restriction is not necessary but it simplifies the arguments and there is no reason to handle a more general situation since we are only interested in the limit as $\Lambda$ and $z$ tend to $\infty$.

Recall that we are calculating

$$
\rho_{a, \lambda} \equiv \lim _{z \rightarrow \infty} \lim _{N \rightarrow \infty} E_{a, \lambda, N}\left(\tau_{z}\right)=\lim _{z \rightarrow \infty} \lim _{N \rightarrow \infty} \frac{S\left(\Phi_{z}^{2} e^{-\int_{G_{s}} d x v\left(\Phi_{x}\right)}\right)}{S\left(e^{-\int_{\mathscr{S}_{s}} d x v\left(\Phi_{x}\right)}\right)},
$$

where

$$
S(\cdot) \equiv \int d \mu_{G_{N}}(\Phi)(\cdot) \bar{\varphi}_{0} \varphi_{0} .
$$

The integrands at every point other than 0 and $z$ can be represented in the form of $I A 1$ while those at the points 0 and $z$ can be represented in the form of $I A 3$, with $d_{2}=1, d_{4}=0$.

Throughout this section we will denote by $U$ a constant independent of induction step, though it may depend on $L$ and $\lambda_{0}$. By $C_{n}$ we denote a constant that may depend on the induction step as well as $L$ and $\lambda_{0}$, but is bounded by some $U$, uniformly in $n$. Either of these symbols can take on different values from formula to formula, or even when they appear twice in the same formula.

At the end of the induction we will see that the final $\beta_{\Lambda}$ will be chosen close to one. Since $\beta_{A}$ increases exponentially throughout the induction, this means that $w_{\Lambda}$ is always close to one. If we choose the volume large enough we see that, in all but the final stage of the induction (after $0, z$ merge), the corrections due to $w_{A}$ are smaller than the error terms we already have. At this point the corrections to the leading terms in the numerator and denominator are the same, so they will cancel. Hence we can ignore the factors of $w_{A}$ here as well.

We proceed in several stages, switching methods as $\beta$, and later $g_{2}$, grow. Let $n$ be the iteration step. The first stage involves applying Propositions 5.2 and 6.2 repeatedly, stopping at the step $n_{1}$ before $\beta_{n}$ becomes larger than $\lambda_{n}^{2}$. We find, for $0 \leqq n \leqq n_{1}$,

$$
\begin{aligned}
\lambda_{n} & =\left(\lambda_{0}^{-1}+\beta_{2} n+O(\log n)\right)^{-1}, \\
\beta_{n} & =\beta_{0} L^{2 n} \exp \left(-\gamma_{\beta} \sum_{i=0}^{n-1} \lambda_{i}\right) \prod_{i=0}^{n-1}\left(1+C_{i} \lambda_{i}^{2}\right) \\
& =\beta_{0} U L^{2 n} n^{-\gamma_{\beta} / \beta_{2}}\left(1+C_{n} \frac{\log n}{n}\right), \\
d_{2}^{(n)} & =d_{2}^{(0)} L^{-2 n} \exp \left(-\gamma_{d} \sum_{i=0}^{n-1} \lambda_{i}\right) \prod_{i=0}^{n-1}\left(1+C_{i} \lambda_{i}^{2}\right) \\
& =d_{2}^{(0)} U L^{-2 n} n^{-\gamma_{d} / \beta_{2}}\left(1+C_{n} \frac{\log n}{n}\right),
\end{aligned}
$$

where we obtain the second form of the expressions for $\beta_{n}$ and $d_{2}^{(n)}$ by inserting the result of the flow of $\lambda_{n}$.

The second stage involves $\lambda_{n}^{2} \leqq \beta_{n}<\sqrt{\lambda_{n}} / 4$, for $n_{1}<n \leqq n_{2}$. Again we apply Proposition 5.2 and 6.2, but now, because the $O\left(\beta_{n} \lambda_{n}\right)$ terms start interfering with the $O\left(\lambda_{n}^{2}\right)$ terms, we do not keep as close track of the subleading terms. Because the 
$\beta_{n}$ are basically increasing exponentially in this regime, the last $O\left(\beta_{n} \lambda_{n}\right)$ term gives the dominant correction:

$$
\lambda_{n_{2}}=\lambda_{n_{1}}+O\left(\lambda_{n_{1}}^{3 / 2}\right) .
$$

To estimate the evolution of $\beta_{n}$ and $d_{2}^{(n)}$ we use

$$
\prod_{i=n_{1}}^{n_{2}-1}\left(1+O\left(\lambda_{n}\right)\right)=1+C_{n_{2}} \lambda_{n_{1}} \log \lambda_{n_{1}}
$$

because, due to the exponential rate of increase of $\beta_{n}$ and the fact that $\lambda_{n}$ is changing very slowly, $n_{2}-n_{1}=O\left(\log \lambda_{n_{1}}\right)$. So

$$
\begin{aligned}
\beta_{n_{2}} & =L^{2\left(n_{2}-n_{1}\right)} \beta_{n_{1}}\left(1+C_{n_{2}} \lambda_{n_{1}} \log \lambda_{n_{1}}\right) \\
& =\beta_{0} U L^{2 n_{2}} n_{1}^{-\gamma_{\beta} / \beta_{2}}\left(1+C_{n_{2}} \frac{\log n_{1}}{n_{1}}\right) \\
d_{2}^{\left(n_{2}\right)} & =L^{-2\left(n_{2}-n_{1}\right)} d_{1}^{\left(n_{1}\right)}\left(1+C_{n_{2}} \lambda_{n_{1}} \log \lambda_{n_{1}}\right) \\
& =d_{2}^{(0)} U L^{-2 n_{2}} n_{1}^{-} \gamma_{d} / \beta_{2}\left(1+C_{n_{2}} \frac{\log n_{1}}{n_{1}}\right),
\end{aligned}
$$

where we have used that $\lambda_{n_{1}}=O\left(n_{1}^{-1}\right)$.

We now switch representations by applying Propositions 5.3 and 6.3, resulting in (primes indicate the new representation)

$$
\begin{aligned}
& \lambda_{n_{2}}^{\prime}=\lambda_{n_{2}}\left(1+O\left(\lambda_{n_{2}}^{1 / 2}\right)\right) \\
& =\lambda_{n_{1}}\left(1+\frac{C_{n_{2}}}{n_{1}^{1 / 2}}\right) \text {, } \\
& A_{n_{2}}^{2}=\frac{1}{2 \lambda_{n_{2}}}\left(\beta_{n_{2}}+\mu_{n_{2}}^{2}-2\left(G^{\left(n_{2}\right)}(0)+a^{\left(n_{2}\right)}\right) \lambda_{n_{2}}\right)+O(1) \\
& =\beta_{0} U L^{2 n_{2}} n_{1}^{1-\gamma_{\beta} / \beta_{2}}\left(1+\frac{C_{n_{2}}}{n_{1}^{1 / 2}}\right) \text {, } \\
& d_{2}^{\left(n_{2}\right)^{\prime}}=L^{-2 n_{2}} d_{2}^{(0)} U n_{1}^{-\gamma_{a} / \beta_{2}}\left(1+\frac{C_{n_{2}}}{n_{1}^{1 / 2}}\right) .
\end{aligned}
$$

Now drop the primes. At this point we start to use $g_{2}$ to parametrize the recursion. We apply Propositions 5.5 and 6.4 up through the last step $n_{3}$ before $g_{2}^{(n)}$ becomes greater than $\lambda_{n}^{1 / 4+v_{s}}$. Again this involves $O\left(\log \lambda_{n_{1}}\right)$ steps. So

$$
\begin{aligned}
\lambda_{n_{3}} & =\lambda_{n_{2}}\left(1+C_{n_{3}} \frac{\log n_{1}}{n_{1}^{1 / 2}}\right), \\
A_{n_{3}}^{2} & =L^{2\left(n_{3}-n_{2}\right)} A_{n_{2}}^{2} \prod_{i=n_{2}}^{n_{3}-1}\left(1+O\left(A_{i}^{-2}\right)\right) \\
& =L^{2\left(n_{3}-n_{2}\right)} A_{n_{2}}^{2}\left(1+\frac{C_{n_{3}}}{n_{1}^{1 / 2}}\right), \\
d_{2}^{\left(n_{3}\right)^{\prime}} & =L^{-2 n_{3}} d_{2}^{(0)} U n_{1}^{-\gamma_{d} / \beta_{2}}\left(1+\frac{c_{n_{3}}}{n_{1}^{1 / 2}}\right) .
\end{aligned}
$$


At this point we start applying Propositions 5.7 and 6.6, which will apply until the volume shrinks to a point in the denominator, and until the points 0 and $z$ are about to merge in the numerator. Denote the former step by $n_{5}$, and the later by $n_{4}$. We see that $g_{2}^{(n)}$ approaches a fixed point of $O\left(L^{2}\right)$ exponentially quickly, and that

$$
\begin{aligned}
A_{n_{4}}^{2} & =L^{2\left(n_{4}-n_{2}\right)} A_{n_{2}}^{2}\left(1+\frac{C_{n_{4}}}{n_{1}^{1 / 2}}\right), \\
d_{2}^{\left(n_{4}\right)} & =L^{-2\left(n_{4}-n_{3}\right)} d_{2}^{\left(n_{3}\right)} \prod_{n=n_{3}}^{n_{4}-1}\left(1+O\left(\frac{g_{2}^{(n)}}{A_{n}}\right)\right) \\
& =L^{-2 n_{4}} d_{2}^{(0)} U n_{1}^{-\gamma_{d} / \beta_{2}}\left(1+\frac{C_{n_{4}}}{n_{1}^{1 / 2}}\right) .
\end{aligned}
$$

For the denominator, replace $n_{4}$ by $n_{5}$. The term $g_{2}^{(n)} / A_{n}$ in the first expression for $d_{2}$ first rises exponentially until $g_{2}=O\left(L^{2}\right)$ and then drops exponentially thereafter. Hence the error term can be estimated by $1+O\left(g_{2}^{(*)} / A_{*}\right)$, where $*$ is the step at which $g_{2}$ first approaches close to the fixed point. Because the fixed point is $O\left(L^{2}\right)$, we see that this error is much smaller than $n_{1}^{-1 / 2}$, which explains the last equality.

All that remains to do for the denominator is to calculate the final onedimensional integral. We will do this below after we have finished the induction for the numerator. At this point in the numerator we have 0 and $z$ in the same block $\mathscr{G}_{1}$. We apply Proposition 6.7 to find

$$
e_{n_{4}+1}=L^{-2\left(n_{4}+1\right)}\left(d_{2}^{(0)}\right)^{2} \beta_{0} U n_{1}^{1-\left(\gamma_{\beta}+2 \gamma_{d}\right) / \beta_{2}}\left(1+\frac{c_{n_{4}+1}}{n_{1}^{1 / 2}}\right) .
$$

Now $e_{n}$ evolves according to Proposition 6.6 again, with the result

$$
e_{n_{5}}=L^{-2 n_{5}}\left(d_{2}^{(0)}\right)^{2} \beta_{0} U n_{1}^{1-\left(\gamma_{\beta}+2 \gamma_{d}\right) / \beta_{2}}\left(1+\frac{c_{n_{5}}}{n_{1}^{1 / 2}}\right) \text {. }
$$

$A_{n_{5}}^{2}$ is given by (7.1) with $n_{4}$ replaced by $n_{5}$.

We have reduced the calculation of $E_{\lambda, N}\left(\tau_{z}\right)$ to the ratio of one-dimensional integrals

$$
E_{\lambda, N}\left(\tau_{z}\right)=\frac{\int d \mu_{G_{0}+a_{0}}\left(\Phi_{0}\right) e^{\beta_{\Lambda}^{\left(n_{5}\right)} \Phi_{0}^{2}}\left(e_{n_{5}} \varphi_{0} \bar{\varphi}_{0} g\left(\Phi_{0}\right)+t_{1}^{\left(e, n_{5}\right)}\left(\Phi_{0}\right)+t_{2}^{\left(e, n_{5}\right)}\left(\Phi_{0}\right)\right)}{\int d \mu_{G_{0}+a_{0}}\left(\Phi_{0}\right) e^{\beta_{\Lambda}^{\left(n_{s}\right)} \Phi_{0}^{2}}\left(d_{2}^{n_{5}} \varphi_{0} \bar{\varphi}_{0} g\left(\Phi_{0}\right)+t_{1}^{\left(d, n_{5}\right)}\left(\Phi_{0}\right)+t_{2}^{\left(d, n_{5}\right)}\left(\Phi_{0}\right)\right)} .
$$

Now, since

$$
d \mu_{G_{0}+a_{0}}\left(\Phi_{0}\right)=d \Phi_{0} e^{-\left(G_{0}+a_{0}\right)^{-1} \Phi_{0}^{2}},
$$

we see that the expedient choice of $\beta_{\Lambda}^{\left(n_{5}\right)}$ is

$$
\beta_{\Lambda}^{\left(n_{5}\right)}=\left(G_{0}+a_{0}\right)^{-1}=1+O\left(L^{-2}\right) .
$$

This substantiates our claim that $w_{A}$ stays close to one throughout the induction. We next apply the following 
Lemma 7.1. If $F(x) \in C^{\infty}\left(\mathbb{R}_{+}\right)$and has exponential decay at $\infty$, then

$$
\begin{aligned}
\int d \Phi F\left(\Phi^{2}\right) & =F(0), \\
\int d \Phi \varphi \bar{\varphi} F\left(\Phi^{2}\right) & =\int_{0}^{\infty} d x F(x) .
\end{aligned}
$$

Proof. Write $F\left(\Phi^{2}\right)=F(\varphi \bar{\varphi})+F^{\prime}(\varphi \bar{\varphi}) \psi \bar{\psi}$ and trace through the definitions of Grassman integration, e.g. Eq. (3.1) in [BEI].

Applying this to the $t_{2}$ terms shows us that these do not contribute in the infinite-volume limit. We apply this to the other remainder term, writing $t_{1}\left(\Phi^{2}\right)=\varphi \bar{\varphi} t_{R}\left(\Phi^{2}\right)$, and apply Corollary 4.7 to bound $t_{R}$, obtaining

$$
\left|t_{R}\left(|\varphi|^{2}\right)\right| \leqq O\left(A^{-2}\right)\left|t_{1}\right|_{K, \alpha g_{2}, A, h} e^{K-\frac{1}{2} \alpha g_{2}(|\varphi|-A)^{2}} .
$$

Also, clearly

$$
\left|r\left(|\varphi|^{2}\right)\right| \leqq|r|_{K, \alpha g_{2}, A, h} e^{K-\alpha g_{2}(|\varphi|-A)^{2}} .
$$

A simple calculation shows that the contributions to the numerator from the $r$ and $t_{1}^{\left(e, n_{5}\right)}$ terms are both $O\left(\sqrt{\lambda_{n_{5}}} A_{n_{5}}\right) e_{n_{5}}$. The contribution from the leading term $e_{n_{5}} \exp \left(-\lambda_{n_{5}}\left(x-A^{2}\right)^{2}\right)$ is $(\sqrt{\pi} / 2) e_{n_{5}} \lambda_{n_{5}}^{-1 / 2}$. Replace $e_{n_{5}}$ by $d_{n_{5}}$ for the denominator in both of these results. Since $\lambda_{n}$ shrinks by a factor of approximately $L^{-2}$ in every step after $g_{2}$ approaches near to the fixed point, while $A_{n}$ increases only by a factor of $L$, we see that the $r$ and $t_{1}$ terms also do not contribute in the infinite volume limit. So, for $\Lambda$ large enough, these remainders are smaller than the ones we already have, and, by inserting (7.3) and (7.2) into (7.4), we see

$$
E_{\lambda, N}\left(\tau_{z}\right)=\beta_{0} U n_{1}^{1-\left(\gamma_{\beta}+\gamma_{d}\right) / \beta_{2}}\left(1+\frac{C_{n_{5}}}{n_{1}^{1 / 2}}\right) .
$$

We now take the limits as $N$, and then $z$, tend to $\infty$, which clearly exist. In order to get the expression in the final form given in Theorem 1.1, we must relate $n_{1}$ to $\beta_{0}$. We do this by noting that, by definition, $\beta_{n_{1}}=O\left(\lambda_{n_{1}}^{2}\right)=O\left(n_{1}^{-2}\right)$. Hence $n_{1}=-U \log \beta_{0}+O\left(\log \log \beta_{0}\right)$, and inserting this into (7.5) yields the desired result.

\section{A. Appendix}

Proof of Lemma 4.3. The only part that does not follow immediately from what we have already done is part (v). For simplicity we handle only the most delicate case of $t=1$. Write $g_{2} \equiv g_{2}^{(n)}, g_{2}^{\prime} \equiv g_{2}^{(n+1)}$, and let $\sigma \equiv|\varphi|-A$. Define

$$
I \equiv \frac{1}{N} \int d \zeta d \bar{\zeta} e^{-(\gamma w)^{-1} \zeta \zeta} e^{-\alpha g_{2}(|\varphi+\zeta|-A)^{2}}
$$

(this is a purely Bosonic integral). From Lemma 4.1, part (ii), we see that if we show that

$$
I \exp \left[\frac{\alpha^{\prime} g_{2}^{(n+1)}}{L^{2} w^{2}}(1+c \lambda) \sigma^{2}\right] \leqq O(1),
$$


then (4.3) will follow. We consider first the case of $g_{2} \leqq \lambda^{1 / 4}$. A calculation shows that (shift $\zeta \rightarrow \zeta-\varphi$ )

$$
I \leqq \frac{O(1)}{N}\left(\frac{(\gamma w)^{-1}|\varphi|+\alpha g_{2} A}{\left[(\gamma w)^{-1}+\alpha g_{2}\right]^{3 / 2}}\right) \exp \left(\frac{-\alpha g_{2}}{1+\alpha g_{2} \gamma w} \sigma^{2}\right)
$$

Insert this in (A.2) and consider the coefficient of $\sigma^{2}$

$$
\begin{aligned}
D \equiv & \frac{\alpha g_{2}}{1+\alpha g_{2} \gamma w}-\frac{\alpha^{\prime} g_{2}^{\prime}}{L^{2} w^{2}}(1+c \lambda) \\
= & \frac{g_{2}\left(\alpha-\alpha^{\prime}+\alpha g_{2} \gamma w\left(1-\alpha^{\prime}\right)+c \lambda\left(\alpha^{\prime}+\alpha \alpha^{\prime} g_{2} \gamma w\right)\right)}{\left(1+\alpha g_{2} \gamma w\right)\left(1+g_{2} \gamma w\right)} \\
& +\left(\alpha^{\prime} / L^{2} w^{2}\right) O\left(g_{2} / A\right)(1+c \lambda) .
\end{aligned}
$$

We note that the $c \lambda$ and $O\left(g_{2} / A\right)$ terms will always be dominated by the first two, and that in the region of small $g_{2}$ (which we are now considering), we have $\alpha-\alpha^{\prime}>\alpha g_{2} \gamma w\left(1-\alpha^{\prime}\right)$. We consider the two regions of small and large $\sigma^{2}$ separately.

In the region $\sigma^{2}>A^{2 \varepsilon} /\left(g_{2}\left(\alpha-\alpha^{\prime}\right)\right)$, with some $\varepsilon>0$ chosen small enough below, the $\exp \left(-g_{2}\left(\alpha-\alpha^{\prime}\right) \sigma^{2}\right)$ dominates the powers in front and we have the desired bound.

Now consider the small-field region of $\sigma^{2} \leqq A^{2 \varepsilon} /\left(g_{2}\left(\alpha-\alpha^{\prime}\right)\right)$. We define $\tilde{\sigma}, \tilde{\pi}$ by $\zeta \equiv \tilde{\sigma} \hat{\varphi}+\tilde{\pi} i \hat{\varphi}$, and

$$
\chi(\tilde{\sigma}, \tilde{\pi}) \equiv \begin{cases}1 & \text { if }|\tilde{\sigma}|,|\tilde{\pi}|<A^{\varepsilon^{\prime}} \\ 0 & \text { otherwise }\end{cases}
$$

with $\varepsilon^{\prime}>\varepsilon$. Insert $\chi+\chi^{c}$ inside the integral (A.1), writing $I=I^{\chi}+I^{c}$. We can estimate $I^{c}$ simply by bounding the exponential inside by 1 and using $\int d \mu_{\gamma w} \chi^{c} \leqq$ $\exp \left(-O\left(A^{2 \varepsilon^{\prime}}\right)\right)$. Also,

$$
\exp \left(\frac{\alpha^{\prime} g_{2}^{\prime}}{L^{2} w}(1+c \lambda) \sigma^{2}\right) \leqq \exp \left(\lambda^{-O(\delta / \log L)} A^{2 \varepsilon}\right)
$$

so by choosing $L$ large enough we can get the requisite bound.

We are left with the $I^{\chi}$ term. For this we define

$$
(|\varphi+\zeta|-A)^{2} \equiv E(\sigma+\tilde{\sigma}, \tilde{\pi})+(\sigma+\tilde{\sigma})^{2}
$$

or, in a more convenient form,

$$
\begin{aligned}
E(\sigma+\tilde{\sigma}, \tilde{\pi})= & \left.\left([A+\sigma+\tilde{\sigma})^{2}+\tilde{\pi}^{2}\right]^{1 / 2}-A+\sigma+\tilde{\sigma}\right) \\
& \left.\times\left([A+\sigma+\tilde{\sigma})^{2}+\tilde{\pi}^{2}\right]^{1 / 2}-A-\sigma-\tilde{\sigma}\right) \\
\equiv & E_{1}(\sigma+\tilde{\sigma}, \tilde{\pi}) E_{2}(\sigma+\tilde{\sigma}, \tilde{\pi}) .
\end{aligned}
$$

We now perform a shift $\tilde{\sigma} \rightarrow \tilde{\sigma}-\alpha g_{2} \gamma w \mathfrak{L} \sigma$, with $\mathfrak{L} \equiv\left(1+\alpha g_{2} \gamma w\right)^{-1}$. Then

$$
I=e^{-\alpha g_{2} \mathfrak{Q} \sigma^{2}} \frac{1}{N} \int d \tilde{\sigma} d \tilde{\pi} e^{-\left((\gamma w)^{-1}+\alpha g_{2}\right) \tilde{\sigma}^{2}-(\gamma w)^{-1} \tilde{\pi}^{2}-\alpha g_{2} E(\mathfrak{I} \sigma+\tilde{\sigma}, \tilde{\pi})} \chi\left(\tilde{\sigma}-\alpha g_{2} \gamma w \mathfrak{L} \sigma, \tilde{\pi}\right) .
$$


Now, it is not hard to see that

$$
\begin{aligned}
& \left|E_{1}(\mathfrak{L} \sigma+\tilde{\sigma}, \tilde{\pi})\right| \leqq O\left(A^{\varepsilon}\left[g_{2}\left(\alpha-\alpha^{\prime}\right)\right]^{-1 / 2}\right) \\
& \left|E_{2}(\mathfrak{L} \sigma+\tilde{\sigma}, \tilde{\pi})\right| \leqq O\left(A^{\varepsilon^{\prime}}\right)
\end{aligned}
$$

where to get the bound on $E_{2}$ it is helpful to note that $A+\mathfrak{L} \sigma=$ $A+\sigma+O\left(g_{2}\right) \sigma=|\varphi|+O\left(g_{2}\right) \sigma$, and $|\varphi| \geqq 0$. Putting these together,

$$
\begin{aligned}
g_{2}|E(\mathcal{Q} \sigma+\tilde{\sigma}, \tilde{\pi})| & \leqq O\left(g_{2}^{1 / 2} A^{\varepsilon+\varepsilon^{\prime}} \lambda^{-O(\delta / \log L)}\right) \\
& \leqq \lambda^{1 / 10}
\end{aligned}
$$

where we have chosen $\varepsilon, \varepsilon^{\prime}$ small enough, and $L$ large enough. Finally,

$$
\begin{aligned}
I & \leqq O(1) e^{-\alpha g_{2} \mathfrak{I} \sigma^{2}} \\
& \leqq e^{\frac{-\alpha^{\prime} g_{2}^{\prime}}{L^{2} w^{2}}(1+c \lambda) \sigma^{2}}
\end{aligned}
$$

which completes the proof for $g_{2} \leqq \lambda^{1 / 4}$.

Next consider the region $\lambda^{1 / 4}<g_{2} \leqq \lambda^{\delta / \log L}$. We keep the same definition of the large-field region of $\sigma^{2}$. The same arguments imply smallness of the large- $\sigma^{2}$ region as well as the $I^{c}$ term. For $I^{\chi}$ we use a new argument, namely we expand $E(\mathfrak{L} \sigma+\tilde{\sigma}, \tilde{\pi})$ in a power series in $(\mathfrak{L} \sigma+\tilde{\sigma}) / A, \tilde{\pi} / A$. In this region, we find

$$
\begin{aligned}
\left|g_{2} E(\mathfrak{L} \sigma+\tilde{\sigma}, \tilde{\pi})\right| & \leqq g_{2} O\left(\tilde{\pi}^{2} \frac{\mathfrak{Q} \sigma+\tilde{\sigma}}{A}, \frac{(\mathfrak{Q} \sigma+\tilde{\sigma})^{4}}{A^{2}}, \frac{\tilde{\pi}^{4}}{A^{2}}\right) \\
& \leqq \lambda^{\varepsilon^{\prime \prime}}
\end{aligned}
$$

for some $\varepsilon^{\prime \prime}>0$, with $\varepsilon, \varepsilon^{\prime}$ small enough and $L$ large enough. This, as before, gives us the bound.

Finally let $g_{2}>\lambda^{\delta} / \ln L$. Here we redefine the large/small- $\sigma^{2}$ boundary to be at $A^{2 \varepsilon} / g_{2}^{2}$, since now the decay comes from the $\alpha g_{2}^{2} \gamma w\left(1-\alpha^{\prime}\right)$ term. Simple arguments like the ones above now yield the desired bounds.

\section{References}

[B] Balaban, T.: A Low Temperature Expansion for Classical N-Vector Models. I. A Renormalization Group Flow. Commun. Math. Phys., to appear

[BEI] Brydges, D., Evans, S.N., Imbrie, J.Z.: Self-Avoiding Walk on a Hierarchical Lattice in Four Dimensions. Ann. Prob. 20, 82-124 (1992)

[BI] Brydges, D., Imbrie, J.Z.: End-to-End Distance for a 4-Dimensional Hierarchical Self-Avoiding Walk. Preprint (1993)

$[\mathrm{dCJ}]$ des Cloizeaux, J., Jannink, G.: Polymers in Solution, Their Modelling and Structure. Oxford: Clarendon Press, 1990

[DS] Duplantier, B., Saleur, H.: Exact Critical Properties of Two-Dimensional Dense Self-Avoiding Walks. Nucl. Phys. B 290 [FS20], 291-326 (1987)

[GK] Gawedzki, K., Kupiainen, A.: Continuum Limit of the Hierarchical $O(N)$ Non-Linear $\sigma$-model. Commun. Math. Phys. 106, 533-550 (1986)

[dG1] de Gennes, P.G.: Collapse of a Polymer Chain in Poor Solvents. J. Phys. L36, L-55-L-57 (1975)

[dG2] de Gennes, P.G.: Scaling Concepts in Polymer Physics. New York: Cornell University Press, 1979 
[G] Gujrati, P.D.: Novel Phase Diagram for Self-Avoiding Walks (Polymers). Phys. Rev. Lett. 55, 1161-1164 (1985)

[IM] Iagolnitzer, D., Magnen, J.: Polymers in a Weak Random Potential in Dimension Four: Rigorous Renormalization Group Analysis. Preprint (1993)

[M] McKane, A.J.: Reformulation of $n \rightarrow 0$ Models Using Anticommuting Scalar Fields. Phys. Lett. A 76, 22-24 (1980)

[MS] Madras, N., Slade, G.: The Self-Avoiding Walk. Boston, Basel, Berlin: Birkhäuser, 1993

[OPB] Owczarek, A.L., Prellberg, T., Brak, R.: New Scaling Form for the Collapsed Polymer Phase. Phys. Rev. Lett. 70, 951-953 (1993)

[PS] Parisi, G., Sourlas, N.: Self-Avoiding Walk and Supersymmetry. J. Phys. L41, L-403L-406 (1980)

[S] Schäfer, L.: Polymer Correlation Functions and Spurious Singularities in $n=0$ Field Theory. Phys. Rev. B 35, 5184-5197 (1987)

[WSPP] Wheeler, J.C., Stilck, J.F., Petschek, R.G., Pfeuty, P.: Parametric Equations of State for the $n$-vector Model and Polymers. Phys. Rev. B 35, 284-296 (1987)

Communicated by D.C. Brydges 
